NBER WORKING PAPER SERIES

\title{
STIRRING UP A HORNETS' NEST: GEOGRAPHIC DISTRIBUTION OF CRIME
}

\author{
Sebastian Galiani \\ Ivan Lopez Cruz \\ Gustavo Torrens \\ Working Paper 22166 \\ http://www.nber.org/papers/w22166 \\ NATIONAL BUREAU OF ECONOMIC RESEARCH \\ 1050 Massachusetts Avenue \\ Cambridge, MA 02138 \\ April 2016
}

This paper is dedicated to the memory of Gary S. Becker. The views expressed herein are those of the authors and do not necessarily reflect the views of the National Bureau of Economic Research.

NBER working papers are circulated for discussion and comment purposes. They have not been peer-reviewed or been subject to the review by the NBER Board of Directors that accompanies official NBER publications.

(C) 2016 by Sebastian Galiani, Ivan Lopez Cruz, and Gustavo Torrens. All rights reserved. Short sections of text, not to exceed two paragraphs, may be quoted without explicit permission provided that full credit, including $\odot$ notice, is given to the source. 
Stirring Up a Hornets' Nest: Geographic Distribution of Crime

Sebastian Galiani, Ivan Lopez Cruz, and Gustavo Torrens

NBER Working Paper No. 22166

April 2016, Revised April 2016

JEL No. K42,R12

\begin{abstract}
This paper develops a model of the geographic distribution of crime in an urban area. When the police protect some neighborhoods (concentrated protection), the city becomes segregated. When the police are evenly deployed across the city (dispersed protection), an integrated city emerges. Unequal societies face a difficult dilemma in that concentrated protection maximizes aggregate welfare but exacerbates social disparities. Taxes and subsidies that can be employed to offset the disadvantages to agents left unprotected. Private security makes an integrated city less likely. Even under dispersed public protection, rich agents may use private security to endogenously isolate themselves in closed neighborhoods.

Sebastian Galiani

Department of Economics

University of Maryland

3105 Tydings Hall

College Park, MD 20742

and NBER

galiani@econ.umd.edu

Ivan Lopez Cruz

University of Indiana

100 South Woodlawn Avenue

Bloomington, Indiana 47405-7104

ilopezcr@indiana.edu

\author{
Gustavo Torrens \\ Department of Economics \\ Indiana University \\ Wylie Hall, 100 S Woodland Ave \\ Bloomington, IN 47405-7104 \\ gtorrens@indiana.edu
}




\title{
Stirring Up a Hornets' Nest: Geographic Distribution of Crime*
}

\author{
Sebastian Galiani \\ University of Maryland
}

\author{
Ivan Lopez Cruz \\ Indiana University
}

April 2016

\author{
Gustavo Torrens \\ Indiana University
}

\begin{abstract}
This paper develops a model of the geographic distribution of crime in an urban area. When the police protect some neighborhoods (concentrated protection), the city becomes segregated. When the police are evenly deployed across the city (dispersed protection), an integrated city emerges. Unequal societies face a difficult dilemma in that concentrated protection maximizes aggregate welfare but exacerbates social disparities. Taxes and subsidies that can be employed to offset the disadvantages to agents left unprotected. Private security makes an integrated city less likely. Even under dispersed public protection, rich agents may use private security to endogenously isolate themselves in closed neighborhoods.
\end{abstract}

JEL classification codes: K42, R12

Keywords: policy deployment, crime, spatial equilibrium, inequality

\section{Introduction}

Following the seminal theoretical work of Becker (1968), a large body of empirical literature has revealed that preventive and punitive measures do in fact have a significant impact on criminal activities (Cook, 2009; Benson and Zimmerman, 2010; Cook et al., 2013; and Draca and Machin, 2015). In particular, regarding law enforcement, it is well established that an increase in the size of the police force reduces crime (Levitt, 1997; Di Tella and Shargrodsky, 2004; Klick and Tabarrok, 2005; Evans and Owens, 2007; and Draca, Machin, and Witt, 2011). The key question now at the frontier of the economics of crime

\footnotetext{
${ }^{*}$ This paper is dedicated to the memory of Gary S. Becker.
} 
and law enforcement is how to make police deployment strategies more efficient (see Benson, 2010 for a recent survey on this topic). This paper aims to help answer this question by developing the first general equilibrium model to study how the geographic distribution of police protection affects the decision to become a criminal, the intensity and location of crime, residential choices, housing prices, and the welfare of different socioeconomic groups. The goal is to study the positive and normative effects of different ways of spatially allocating police forces in a city.

Section 2 develops a baseline model. The building block is a model of a city populated by agents of different socioeconomic groups and made up of several residential areas, which are denoted as neighborhoods. Socioeconomic groups are distinguished by their factor endowments. In particular, there is one homogenous group of skilled agents, and several homogenous groups of unskilled agents, each of them with a different per capita endowment of unskilled labor. The city is treated as a small open economy, that is, the prices of tradable goods and inputs are exogenously given. Agents select (1) their occupation (i.e., work in firms that produce goods or become a criminal); (2) their residence (i.e., in which neighborhood to reside); and (3) consumption and housing. Firms demand unskilled and skilled labor and supply tradable goods using a constant returns to scale technology. Criminals use their labor endowments to extract income from other citizens. The supply of housing in each neighborhood is chosen by profit-maximizer developers, who use capital and land to build houses. Capital is a tradable input, that is, it is elastically supplied at a given price, while land is a nontradable input in fixed supply in each neighborhood. The government provides public protection by deploying police forces in the city, which reduces the amount that criminals can otherwise extract from their victims. Given the prices of tradable goods and inputs and the allocation of public protection, the model determines the three endogenous variables mentioned above by employing a combination of a standard small and open-economy competitive equilibrium and a spatial notion of equilibrium. In equilibrium, no agent can obtain a rent changing his or her occupation and/or location. Thus, we adopt a long-run perspective that allows agents enough time to change their occupation and residence.

The central object of the analysis is public protection regimes. We consider two extreme strategies to allocate the police across the city, which we assume are both feasible and have the same cost. Under concentrated public protection, the police only protect some neighborhoods and leave the rest of the city completely unprotected. Under dispersed public protection, the police are evenly deployed across the 
entire city, inducing an equal level of public protection in all neighborhoods. With these two protection regimes we try to capture, albeit in stylized fashion, the basic trade-off faced by the police with regard to the geographic allocation of protection. With the same resources, the police can either extensively protect a smaller area (concentrated protection) or partially protect a larger area (dispersed protection).

Section 3 characterizes the equilibrium in each protection regime. It shows that, under proper conditions, concentrated public protection leads to a spatially segregated city. Only rich agents are willing to pay the high housing prices in protected neighborhoods, while poor workers and criminals reside in unprotected neighborhoods. Neighborhoods protected by the police are safe, concentrate wealthy citizens, and have high land and housing prices. In the rest of the city, crime is pervasive, neighborhoods are populated by poor agents, and land and housing prices are relatively low. The opposite happens under dispersed public protection. When the police force is evenly dispersed across all neighborhoods, the city becomes fully integrated. All neighborhoods are inhabited by citizens of all income levels. Indeed, income per capita and crime levels are equalized across the city.

There are two mechanisms operating in the model presented here that produce these results. Regarding occupational choices, the payoff from crime does not vary with a citizen's labor endowment, while the payoff from working is obviously increasing in a citizen's labor endowment. This makes relatively poor citizens more prone to become criminals. Indeed, both under concentrated and dispersed public protection, we focus on a region of the parameter space for which, in equilibrium, only agents in the poorest socioeconomic group decide to become criminals. Regarding residential choices, the wealthier the agents, the more harmful criminal activities are for them and, as a consequence, the more they are willing to sacrifice in order to avoid high-crime areas. Under concentrated public protection, these differences in the willingness to pay for a safe neighborhood produce a concentration of rich agents in protected neighborhoods and poor agents in unprotected neighborhoods. Under dispersed public protection, there is no essential difference among neighborhoods, crime distributes evenly in the city, and agents only take into account housing prices in their residential choices. As a consequence, there is housing price equalization across the city and all neighborhoods have the same income per capita.

After formally characterizing the equilibrium under concentrated and dispersed protection, Section 4 makes several comparisons. In particular, it compares crime, measured as the total income of criminals, aggregate income, and housing prices. With respect to crime, there is likely to be more of it under 
dispersed protection than under concentrated protection when the wage-income share of skilled agents is high, dispersing the police significantly reduces its effectiveness, the proportion of income that criminals can extract from rich agents is high, and the proportion of income that criminals can extract from the poor is low. Aggregate income is more likely to be higher under concentrated protection, the higher the wage-income share of skilled agents, the more intense the reduction in police effectiveness when the force is dispersed, the more criminals can extract from skilled workers, and the less criminals can extract from unskilled workers. Finally, we find conditions under which housing prices under dispersed protection are higher than housing prices in unprotected neighborhoods but lower than housing prices in protected neighborhoods under concentrated protection. We also show that these conditions are more likely to be satisfied the higher the wage-income share of skilled agents.

Section 4 also examines the welfare and distributive effects associated with a change in the public protection regime. First, employing a simple utilitarian welfare function, we show that concentrated protection may induce higher aggregate welfare than dispersed protection. Moreover, income inequality matters. We prove that aggregate welfare is higher under concentrated protection for a society with a high wage-income share of skilled agents, while aggregate welfare is higher under dispersed protection for a society with a low wage-income share of skilled agents.

Second, regarding distributive effects, we prove that, with regard to a utilitarian welfare function, unskilled agents as a whole are better off under dispersed protection. Thus, societies with high levels of income inequality may face a complicated dilemma. Concentrated protection may maximize aggregate welfare but exacerbate social disparities. In contrast, in more equalitarian societies, dispersed protection simultaneously maximizes aggregate welfare and reduces social disparities. One solution to the regressive distributive consequences of concentrated protection is to supplement the regime with a set of taxes and transfers. We deduce a set of equations for the compensation that must be provided in order to make each unskilled agent equally well off between concentrated and dispersed protection. Conceptually, the compensation received by each socioeconomic group takes into account the impact of the protection regime on income (different for each group) and housing prices (common for all groups). However, there are general equilibrium effects and, therefore, each compensation cannot be computed separately.

Third, we explore some interesting political economy implications related to the distributive effects of a change in the public protection regime. In particular, there is room for the formation of atypical 
political coalitions with respect to public safety. Although at least one group of unskilled agents will be better off under dispersed than under concentrated protection, it is not necessarily the case that all unskilled agents unanimously prefer dispersed protection. Dispersing police forces could induce an increase in the housing prices paid by unskilled workers that consumes their gains in income. Thus, skilled and some unskilled agents may have an incentive to form a coalition that supports concentrated protection.

Section 5 explores two extensions to the baseline model. First, it introduces private security. In many countries different forms of private security are intensively employed by households and businesses. In principle, our baseline model can handle pure private security such us alarm systems and security doors. One alternative is to reinterpret the fraction of income that criminals extract from other agents as the outcome of a more detailed game that incorporates defensive measures by the victims. More interesting are closed neighborhoods protected by fences and security guards or patrols collectively financed by the neighbors - that is, when private security works as a club good. In this case, we show that dispersed public protection does not necessarily lead to an integrated city equilibrium in which crime, income per capita, and housing prices are spatially equalized. The reason is that skilled agents may use private protection to endogenously isolate themselves in fully protected areas, while high housing prices exclude unskilled workers from these areas.

Second, the section considers a model with a continuum of agent types. Instead of four socioeconomic groups, we assume that the distribution of labor endowments in the population is given by a continuous distribution function over an interval. Although numerical solutions are required to obtain a full characterization of the equilibrium of the model with a continuum of agent types, we find conditions under which concentrated public protection leads to a segregated city equilibrium, while dispersed public protection induces an integrated city equilibrium. Thus, the structure of the equilibrium associated with each protection regime is the same as in the baseline model. In both protection regimes, in equilibrium, only agents with labor endowments below some occupational threshold become criminals (although the thresholds are generally different in each regime). Under concentrated protection, agents with labor endowments below a residential threshold reside in unprotected neighborhoods with low housing prices, while agents above the threshold reside in protected neighborhoods with high housing prices. Under dispersed protection, agents of all types reside in every neighborhood and housing prices are equalized 
across the city. Regime comparisons are more complicated in the model with a continuum of agent types because they depend on the occupational and residential thresholds, which must be computed numerically. Nevertheless, we show that in order to compare aggregate welfare, we only need to compute the occupational and residential thresholds for the equilibrium under concentrated protection. This is interesting because it indicates that crime statistics could be a misleading way to infer welfare changes.

There are two bodies of literature connected with this work. First, there is a vast literature studying how police presence can deter crime and disorder. Various mechanisms have been identified, from influencing the probability of apprehension of active offenders, which is a necessary step for their subsequent conviction, to proactively targeting "hot" places and people, responding more rapidly to calls for services, and conducting successful post-crime investigation (Glaeser, 1999; Levitt, 2004; Yezer, 2014; and Lum and Nagin, 2015). Surprisingly, to the best of our knowledge, this paper is the first to analytically study the consequences of police deployment strategies on the endogenous formation of urban spatial equilibria and crime. Banerjee et al. (2002) conduct a RCT to examine the optimal composition of deterrence interventions in India. Fu and Wolpin (2015) estimate a structural model of crime using MSA-level data and employ their estimations to evaluate several targeting schemes that allocate federally-sponsored additional police across cities. While they focus on the allocation of additional police across cities, we study police deployment strategies within a city. As a consequence, we do not only model occupational choices but also the location of criminal activities, residential choices and housing markets. In a very different context, Quy-Toan, Ma and Ruiz (2016) study two crime deterrence strategies to combat Somali piracy: the deployment of international navies in pirate-infested waters and the provision of armed security guards onboard vessels.

Second, there is a large literature on urban economics, especially the notion of spatial equilibrium (see, for example, Glaeser, 2008) as well as on spatial and socioeconomic segregation (see, for example, Benabou, 1993, 1996, Verdier and Zenou, 2004 and Calabrese et. al., 2006). We extensively use the idea that, in a spatial equilibrium, the location of both legal and illegal activities must be such that no agent has an incentive to move his or her activities to a different location. Indeed, we apply to the geographic distribution of crime the same tools many urban economists have used to explain the location of legal activities. Following this parallelism, just as public education policies, highways and other public infrastructure affect the incentives of households and businesses to locate in different areas 
of the city, we show that police deployment strategies shape the geographic distribution of legal and illegal economic activities. As do many studies on urban economics and socioeconomic segregation, our model also emphasizes the importance of properly accounting for changes in housing prices. In particular, we stress two channels through which police deployment strategies affect the welfare of each individual. First, they affect the intensity and location of crime, thus affecting the income of each agent; and second, they induce changes in housing prices.

The rest of the paper is organized as follows. Section 2 develops the baseline model. Section 3 characterizes the equilibrium under concentrated and dispersed protection. Section 4 compares the two protection regimes. Section 5 develops two extensions. First, we introduce private security. Second, we explore a model with a continuum of agent types. Finally, Section 6 concludes.

\section{A Model of Crime and Residence}

This section develops a simple model of the geographic distribution of crime and residence in a city, which we treat as a small open economy. Citizens select their occupation and area of residence. The government provides police protection. The distribution of agents to occupations and residential areas, as well as wages, housing, and land prices, is all determined in equilibrium.

\subsection{Agents, Neighborhoods, and Endowments}

Consider a city integrated by a finite set of neighborhoods indexed by $j \in J$. Each neighborhood has a fixed quantity of land $\bar{L}_{j}$ that can be used to build houses. Let $\bar{L}=\sum_{j \in J} \bar{L}_{j}$ be the total quantity of land for residential purposes. The city is populated by four homogeneous socioeconomic groups indexed by $i \in I=\{l, m, h, s\}$ : three groups of unskilled agents denoted by $l, m$, and $h$, and a group of skilled agents denoted by $s$. The mass of people in group $i$ is $N^{i}$. Each unskilled agent owns $\bar{e}^{i}$ units of unskilled labor, where $0 \leq \bar{e}^{l}<\bar{e}^{m}<\bar{e}^{h}$, while each skilled agent owns $\bar{e}^{s}$ units of skilled labor and an equal share of all land. 


\subsection{Residence and Occupations}

Each agent has a residential choice. Let $r^{i} \in J$ be the residential choice of an agent in group $i$. Each agent also has an occupational choice. The agent can be a worker denoted by $w$, that is, the agent can rent his or her labor endowment to producers in exchange for a wage. In such a case, let $w^{u}$ and $w^{s}$ be the wage rates of unskilled and skilled labor, respectively. Alternatively, the agent can become a criminal who operates in neighborhood $j$ (denoted by $b_{j}$ ), that is, the agent can use his or her labor endowment to extract resources from workers residing in $j$. Let $o^{i} \in O=\left\{w, b_{1}, \ldots, b_{J}\right\}$ indicate the occupational choice of an agent in group $i$.

The income of an agent depends on his or her occupation and residence. Let $y^{i}\left(o^{i}, r^{i}\right)$ indicates the income of an agent in group $i$ who selects $\left(o^{i}, r^{i}\right)$. For a worker in group $i$ who resides in $j$, his or her income is given by $y^{i}(w, j)=\left(1-\gamma_{j}^{i}\right)\left[w^{i} \bar{e}^{i}+s_{L}^{i} \sum_{j \in J} p_{L, j} \bar{L}_{j}\right]$, where $\gamma_{j}^{i} \in[0,1]$ is the fraction extracted by criminals, $s_{L}^{i}=0$ for $i=l, m, h$ and $s_{L}^{s}=1 / N^{s}$ (all land is evenly owned by skilled workers) and $p_{L, j}$ is the price of a unit of land in neighborhood $j$. Thus, each worker only keeps a fraction $\left(1-\gamma_{j}^{i}\right)$ of his market income. Later we will further specify $\gamma_{j}^{i}$. For the moment, note that $\gamma_{j}^{i}$ may vary with the group $i$ and the neighborhood $j$.

Let $N^{i}\left(o^{i}, r^{i}\right)$ denote the mass of agents in group $i$ who select $\left(o^{i}, r^{i}\right)$. Then, for an agent in group $i$ who selects $b_{j}$ his or her income is given by $y^{i}\left(b_{j}\right)=\frac{\delta \sum_{i \in I} \gamma_{j}^{i} N^{i}(w, j)\left[w^{i} \bar{e}^{i}+s_{L}^{i} \sum_{j \in J} p_{L, j} \bar{L}_{j}\right]}{\sum_{i \in I} \sum_{r^{i} \in J} N^{i}\left(b_{j}, r^{i}\right)}$, where $\delta \in(0,1)$. The numerator is the total plunder in neighborhood $j$ after substracting the fraction of goods lost due to criminal activities. Indeed, only a fraction $\eta_{j}^{i}=1-\gamma_{j}^{i}+\delta \gamma_{j}^{i}$ out of each unit of income of a worker in group $i$ who resides in neighborhood $j$ survives the destruction that crime provokes. The denominator is the mass of criminals operating in neighborhood $j$. Thus, each criminal who operates in $j$ obtains an equal share of the total plunder in $j$. Note that criminals do not rob each other. As a consequence, the income of criminals depend on where they decide to operate, but not on their residential choice.

\subsection{Preferences}

All agents derive utility from a consumption good and housing services. Specifically, they all have identical Cobb-Douglas preferences, that is, the utility of an agent of group $i$ who selects occupation $o^{i}$, place of residence $r^{i}$, consumption level $c_{r^{i}}^{i}$ and housing services $h_{r^{i}}^{i}$ is given by $v^{i}\left(o^{i}, r^{i}, h_{r^{i}}^{i}, c_{r^{i}}^{i}\right)=$ 
$\left(\frac{h_{r i}^{i}}{\alpha}\right)^{\alpha}\left(\frac{c_{r i}^{i}}{1-\alpha}\right)^{1-\alpha}$, with $0<\alpha<1$. Let $p_{C}$ denotes the price of the consumption good and $p_{H, j}$ the price of housing services in neighborhood $j$. Then, the budget constraint of an agent in group $i$ is given by $p_{H, r_{i}} h_{r^{i}}^{i}+p_{C} c_{r^{i}}^{i}=y^{i}\left(o^{i}, r^{i}\right)$.

\subsection{Production and Trade}

Production is undertaken by competitive firms in both industries: consumption goods and housing. The consumption industry employs unskilled and skilled workers. Specifically, assume a Cobb-Douglas production function $Q_{C}=\left(U_{C}\right)^{\beta_{U, C}}\left(S_{C}\right)^{1-\beta_{U, C}}$, where $U_{C}$ and $S_{C}$ are the amount of unskilled and skilled labor employed, respectively, and $\beta_{U, C} \in(0,1)$. Developers use land and capital to build houses. Again, assume a Cobb-Douglas production function $Q_{H, j}=\left(K_{H, j}\right)^{\beta_{K, H}}\left(L_{j}\right)^{1-\beta_{L, H}}$, where $K_{H, j}$ stands for capital used in the construction of houses in neighborhood $j$ and $\beta_{K, H} \in(0,1)$. We assume that the city is a small open economy. The consumption good is a tradable final good, while houses are nontradable final goods. Capital is a tradable input, while all other inputs (unskilled and skilled labor and land) are nontradable. The city takes the price of tradable goods $p_{C}=\bar{p}_{C}>0$ and $p_{K}=\bar{p}_{K}>0$ as given. Moreover, we assume balanced trade, that is, $\bar{p}_{K} \sum_{j \in J} K_{H, j}=\bar{p}_{C}\left(Q_{C}-C_{C}\right)$.

\subsection{Police Protection}

The government's role is restricted to providing police protection to the neighborhoods. Assume that the police budget is given and consider two protection strategies of equal cost: concentrated police protection and dispersed police protection. Under concentrated protection, the police only protect some neighborhoods. As a consequence, the residents of those neighborhoods are fully protected against crime, while the residents of other neighborhoods suffer the full brunt of criminal activities. Under dispersed protection, the police force is evenly deployed in all neighborhoods, inducing equal levels of protection throughout. ${ }^{1}$ Formally, $\gamma_{j}^{i}=\left(1-p_{j}\right) \gamma^{i}$, where $\gamma^{i} \in(0,1)$ is the fraction extracted by criminals from an agent of group $i$ when there is no police protection and $p_{j}$ represents the protection strategy. Under concentrated protection, $p_{j}=1$ for $j \in J_{p} \subset J$ (protected neighborhoods) and $p_{j}=0$ for $j \in J_{u n}=J-J_{p}$ (unprotected neighborhoods). Under dispersed protection, $p_{j}=p<1$ for all $j \in J$. Thus, under

\footnotetext{
${ }^{1}$ Concentrated and dispersed protection should not be confused with deterministic and random policing. As Eeckhout, Persico and Todd (2010) show random crackdowns can be part of the police optimal monitoring strategy.
} 
concentrated protection, $\gamma_{j}^{i}=0$ for $j \in J_{p}$ and $\gamma_{j}^{i}=\gamma^{i}$ for $j \in J_{u n}$, while under dispersed protection, $\gamma_{j}^{i}=(1-p) \gamma^{i}$ for $j \in J$. Note that $p$ can be interpreted as the reduction in the effectiveness of protection when the police force is dispersed. ${ }^{2}$

\subsection{Crime and Income Distribution}

We assume that wealthier agents are more vulnerable to crime. However, we put a limit on how much crime reshapes income distribution. In particular, we keep the income ranking among the socioeconomic groups constant, before and after crime enters the picture.

Assumption 1: The proportion of income that criminals extract from a worker is higher the richer the worker is. Formally:

$$
\gamma^{s}>\gamma^{h}>\gamma^{m}>\gamma^{l}
$$

However, this is not enough to affect the ranking of groups. Formally:

$$
\left[\frac{\left(1-\beta_{U, C}\right) N^{h} \bar{e}^{h}}{\beta_{U, C} N^{s} \bar{e}^{s}}\right] \bar{e}^{s}\left(1-\gamma^{s}\right)>\bar{e}^{h}\left(1-\gamma^{h}\right)>\bar{e}^{m}\left(1-\gamma^{m}\right)>\bar{e}^{l}\left(1-\gamma^{l}\right) .
$$

Assumption 1 will be important and, hence, requires a more detailed discussion. First, note that Assumption 1 does not take into account any protection (either public or private). It imposes restrictions on the effects of criminal activities in a hypothetical world without public protection. Second, a simple way to justify condition (1) is to consider the incentives of criminals to target workers with different levels of income as in Becker's (1968) seminal model of crime. Suppose that each time they mount a robbery, criminals manage to extract a fraction $\gamma$ of the victim's income, regardless of the victim's income level. If there is any observable attribute that signals victims' income, then criminals will attack rich agents more frequently. If we interpret $\gamma^{i}$ as the fraction extracted from an agent during a period of time, which is consistent with our model, then condition (1) holds. Indeed, even if each time they are robbed poor agents lose a higher fraction of their income than rich agents, it is still possible that rich agents will be

\footnotetext{
${ }^{2}$ It is possible to consider more moderate policies. For example, assume that under concentrated protection, $p_{j}=p_{H}$ for $j \in J_{p}$ and $p_{j}=p_{L}$ for $j \in J-J_{p}$, while under dispersed protection, $p_{j}=p_{H}^{\prime}$ for $j \in J_{p}$ and $p_{j}=p_{L}^{\prime}$ for $j \in J-J_{p}$, where $p_{H}>p_{H}^{\prime}>p_{L}^{\prime}>p_{L}$. The qualitative results of the paper will not be affected. It is also possible to consider that $p_{j}$ depends on the size of the police force relative to the mass of criminals. In particular, given a fixed police force, it is reasonable to assume that $p_{j}$ decreases with the mass of criminals. This will not affect our results.
} 
targeted more frequently and, as a consequence, over a long enough period of time will end up losing a higher fraction of their income. ${ }^{3}$ More generally, the key necessary assumption for our results is that the richer an agent, the more the agent is willing to pay for security. In this sense, condition (1) can be seen as a natural sufficient condition to induce the required order on the willingness to pay for security in our model.

Third, crime affects income distribution, but does not reverse the initial ranking of socioeconomic groups. In order to see this, note that the income of an agent in group $i=l, m, h$ who chooses to be a worker and suffers the maximum possible extraction $\gamma^{i}$ is $w^{u} \bar{e}^{i}\left(1-\gamma^{i}\right)$. Thus, the last three inequalities in condition (2) mean that workers in group $h$ are richer than workers in group $m$, who are richer than workers in group $l$, even after we take into account crime. The wage-income of an agent in group $s$ who chooses to be a worker and suffers the maximum possible extraction is $w^{s} \bar{e}^{s}\left(1-\gamma^{s}\right)$. With the Cobb-Douglas production function, it must be the case that $w^{s}=w^{u}\left[\left(1-\beta_{U, C}\right) U_{C} / \beta_{U, C} S_{C}\right]$. In the worst possible case where only agents in group $s$ and $h$ choose to be workers, we have $w^{s}=$ $w^{u}\left[\left(1-\beta_{U, C}\right) N^{h} \bar{e}^{h} / \beta_{U, C} N^{s} \bar{e}^{s}\right]$. Thus, the first inequality in condition (2) implies that skilled workers are richer than unskilled workers.

\subsection{Equilibrium Conditions}

Given the police protection allocation $\left(p_{j}\right)_{j \in J}$ and the prices of tradable goods $p_{C}=\bar{p}_{C}>0$ and $p_{K}=\bar{p}_{K}>0$, an equilibrium is: (1) a price vector $\left(w^{u}, w^{s},\left(p_{H, j}, p_{L, j}\right)_{j \in J}\right)$ (i.e., factor rewards and prices of nontradable goods); (2) a production vector $\left(Q_{C},\left(Q_{H, j}, K_{H, j}\right)_{j \in J}\right)$; (3) occupational and residential decisions $\left(U_{C}, S_{C}, N^{i}\left(o^{i}, j\right)_{i \in I, j \in J}\right)$; and (4) income levels $\left(y^{i}\left(o^{i}, j\right)\right)_{i \in I, j \in J}$ such that: (1) Given the price vector $\left(w^{u}, w^{s},\left(p_{H, j}, p_{L, j}\right)_{j \in J}\right)$ and occupational and residential decisions $N^{i}\left(o^{i}, j\right)$, each agent maximizes his or her utility: $y^{i}\left(o^{i}, r^{i}\right) /\left(p_{H, r^{i}}\right)^{\alpha}\left(p_{C}\right)^{1-\alpha} \geq$

\footnotetext{
${ }^{3}$ For evidence consistent with this assumption, see, among others, Demombynesa and Ozlerb (2005) and Di Tella, Galiani and Schargrodsky (2010). In particular, Demombynesa and Ozlerb (2005) show that criminals move to rich neighborhoods to steal from the rich while Di Tella, Galiani and Schargrodsky (2010) show that when crime is low, the rich do not protect themselves and they are the main target of criminals while when crime is very high, they respond by buying private protection displacing crime towards the poor. Another, very different way to justify condition (1) is to rely on Hirshleifer's (1991) paradox of power. Suppose we interpret $\gamma^{i}$ as the outcome of a more primitive conflict game under anarchy. Poor agents will have an advantage in this game and, hence, conflict will mitigate income inequality.
} 
$y^{i}(o, r) /\left(p_{H, r}\right)^{\alpha}\left(p_{C}\right)^{1-\alpha}$ for all $(o, r)$. (2) Given the price vector $\left(w^{u}, w^{s},\left(p_{H, j}, p_{L, j}\right)_{j \in J}\right)$, producers and developers maximize profits: $w^{u}=p_{C} \beta_{U, C}\left(S_{C} / U_{C}\right)^{1-\beta_{U, C}}, w^{s}=p_{C}\left(1-\beta_{U, C}\right)\left(U_{C} / S_{C}\right)^{\beta_{U, C}}$, $K_{H, j}=\beta_{K, H} p_{L, j} \bar{L}_{j} /\left(1-\beta_{K, H}\right) / p_{K}, p_{H, j}=\left(p_{K} / \beta_{K, H}\right)^{\beta_{K, H}}\left(p_{L, j} /\left(1-\beta_{K, H}\right)\right)^{1-\beta_{K, H}}$, and $p_{H, j} Q_{H, j}=$ $p_{L, j} \bar{L}_{j} /\left(1-\beta_{K, H}\right)$. (3) Housing markets clear: $p_{H, j} Q_{H, j}=\alpha \sum_{i \in I} \sum_{o^{i} \in O} N^{i}\left(o^{i}, j\right) y^{i}\left(o^{i}, j\right)$. (4) Labor markets clear: $U_{C}=\sum_{i=l, m, h} \sum_{r^{i} \in J} N^{i}\left(w, r^{i}\right) \bar{e}^{i}$ and $S_{C}=\sum_{r^{i} \in J} N^{s}\left(w, r^{i}\right) \bar{e}^{s}$. (5) Income levels are given by: $y^{i}(w, j)=\left(1-\gamma_{j}^{i}\right)\left[w^{i} \bar{e}^{i}+s_{L}^{i} \sum_{j \in J} p_{L, j} \bar{L}_{j}\right]$ and $y^{i}\left(b_{j}\right)=$ $\delta\left[\sum_{i \in I} \sum_{r^{i} \in J} N^{i}\left(b_{j}, r^{i}\right)\right]^{-1} \sum_{i \in I} \gamma_{j}^{i} N^{i}(w, j)\left[w^{i} \bar{e}^{i}+s_{L}^{i} \sum_{j \in J} p_{L, j} \bar{L}_{j}\right]$.

There are two important features of this notion of equilibrium. First, individuals sort themselves across neighborhoods and select occupations in such a way that there are no gains associated with changes in their decisions. Second, the city is a small open economy for which the prices of tradable goods are exogenously given. Factor prices and the prices of nontradable goods are determined in competitive markets.

\section{Equilibrium}

This section characterizes the equilibrium of the model under concentrated and dispersed protection, respectively. To facilitate the characterization, the section examines a region of the parameter space in which the protection regime does not affect occupational choices. In particular, we impose conditions such that under both concentrated and dispersed protection, in equilibrium, only agents in group $l$ become criminals.

\subsection{Concentrated Protection}

Suppose that police protection is concentrated in some neighborhoods of the city. Specifically, assume that for security purposes the city is divided into two set of neighborhoods, $J_{p} \subset J$ and $J_{u n}=J-J_{p}$. Neighborhood $j \in J_{p}$ is fully protected by the police, while neighborhood $j \in J_{u n}$ is left entirely unprotected. Let $\bar{L}_{p}=\sum_{j \in J_{p}} \bar{L}_{j}$ and $\bar{L}_{u n}=\sum_{j \in J_{u n}} \bar{L}$ be the land in protected and unprotected neighborhoods, respectively. In equilibrium, no criminal activity arises in $J_{p}$. If there are any criminals, they must locate in $J_{u n}$. An agent exposed to crime keeps a fraction $1-\gamma^{i}$ of his or her income and criminals retain $\delta \gamma^{i}$ 
of the victim's income. Since the payoff of criminal activities depends on the income of others, and the poorer an agent the lower the opportunity cost of crime, only poor agents become criminals.

Regarding residential decisions, there are two neighborhood characteristics that agents weigh: housing prices and local protection. Moreover, in equilibrium, neighborhoods with the same level of protection have the same housing and land prices. Formally, $p_{H, j}=p_{H_{p}}$ and $p_{L, j}=p_{L_{p}}$ for $j \in J_{p}$ and $p_{H, j}=p_{H_{u n}}$ and $p_{L, j}=p_{L_{u n}}$ for $j \in J_{u n}$. The wealthier an agent, the more harmful criminal activities are for him or her and, hence, the more prone he or she is to avoid neighborhoods in $J_{u n}$ and choose to reside in neighborhoods in $J_{p}$. Summing up, when protection is concentrated, criminals only operate and reside in $J_{u n}$ and their victims are poor workers who also reside in $J_{u n}$, while rich workers reside in $J_{p}$. It remains only to find the marginal agents, that is, we need to determine the wealthiest agent who prefers to become a criminal and the wealthiest agent who prefers to reside in $J_{u n}$.

Assume that all skilled agents reside in $J_{p}$, all unskilled agents reside in $J_{u n}$, and only agents in group $l$ become criminals. Skilled agents will not have an incentive to change residence if and only if $1-\gamma^{s}<\left(p_{H_{u n}} / p_{H_{p}}\right)^{\alpha}=\left(p_{L_{u n}} / p_{L_{p}}\right)^{\alpha\left(1-\beta_{K, H}\right)}$, that is, when lower housing prices in $j \in J_{u n}$ are not enough to compensate them for the income they lose due to criminal activities in such neighborhoods. Moreover, since $p_{H, j}=p_{H_{p}}$ for $j \in J_{p}$, the proportion of skilled agents who reside in $j \in J_{p}$ is $\bar{L}_{j} / \bar{L}_{p}$. Unskilled workers will not have an incentive to change residence if and only if $1-\gamma^{h}>\left(p_{H_{u n}} / p_{H_{p}}\right)^{\alpha}=$ $\left(p_{L_{u n}} / p_{L_{p}}\right)^{\alpha\left(1-\beta_{K, H}\right)}$, that is, when lower housing prices in $j \in J_{u n}$ are more than enough to compensate unskilled workers for the income they lose due to criminal activities in such neighborhoods. Since $\gamma^{h} \geq$ $\gamma^{m}>\gamma^{l}$ (due to Assumption 1), agents in groups $m$ and $l$ also prefer to reside in $J_{u n}$. Moreover, since $p_{H, j}=p_{H_{u n}}$ for $j \in J_{u n}$, the proportion of unskilled workers in group $i$ who reside in $j \in J_{u n}$ is $\bar{L}_{j} / \bar{L}_{u n}$. Finally, only agents in group $l$ become criminals who operate in $j \in J_{u n}$ if and only if $\left(1-\gamma^{l}\right) w^{u} \bar{e}^{l}<$ $y^{l}\left(b_{j}\right)<\left(1-\gamma^{m}\right) w^{u} \bar{e}^{m}$, where $y^{l}\left(b_{j}\right)=\delta w^{u}\left(N^{l}\right)^{-1}\left(\gamma^{m} N^{m} \bar{e}^{m}+\gamma^{h} N^{h} \bar{e}^{h}\right)$ is the income of a criminal when only agents in group $l$ are criminals. Since $\bar{e}^{h}\left(1-\gamma^{h}\right)>\bar{e}^{m}\left(1-\gamma^{m}\right)$ (due to Assumption 1), agents in group $h$ prefer to be workers as well. Note that, in equilibrium, criminals distribute in $J_{u n}$ proportionally to the land of each neighborhood, that is, the proportion of criminals who operate in $j \in J_{u n}$ is $\bar{L}_{j} / \bar{L}_{u n}$.

$p_{L_{p}}$ and $p_{L_{u n}}$ are endogenous variables that depend on housing demand and supply in each neighborhood. However, given occupational and residential decisions we can easily compute aggregate income 
in each neighborhood and, hence, equilibrium housing and land prices. Intuitively, $p_{L_{u n}} / p_{L_{p}}$ depends on the ratio of aggregate incomes in relation to the ratio of supply of land in protected and unprotected neighborhoods. Finally, we must verify that no agent prefers to change his or her original residential choice for those equilibrium prices, that is, we need $1-\gamma^{s}<\left(p_{L_{u n}} / p_{L_{p}}\right)^{\alpha\left(1-\beta_{K, H}\right)}<1-\gamma^{h}$.

Lemma 1 formally characterizes the equilibrium under concentrated protection. Let $n^{i}=$ $\bar{e}^{i} N^{i}\left(\sum_{i=m, h} \bar{e}^{i} N^{i}\right)^{-1}$ be group $i$ 's labor endowment relative to the labor endowment of groups $m$ and $h$. Define $\eta_{C}^{i}=1-\gamma^{i}+\delta \gamma^{i}$ and $\hat{\eta}_{C}^{i}=\frac{\beta_{U, C} \alpha\left(1-\beta_{K, H}\right) \eta_{C}^{i}+\left(1-\beta_{U, C}\right)}{\beta_{U, C}\left[1-\alpha\left(1-\beta_{K, H}\right)\right]}$.

Lemma 1 Concentrated Protection. Suppose that protection is concentrated, Assumption 1 holds and the following conditions are satisfied:

$$
\begin{aligned}
\left(1-\gamma^{l}\right) \bar{e}^{l} & <\delta\left(N^{l}\right)^{-1} \sum_{i=m, h} \gamma^{i} N^{i} \bar{e}^{i}<\left(1-\gamma^{m}\right) \bar{e}^{m} \\
\left(1-\gamma^{s}\right) & <\left(\frac{\bar{L}_{p} \sum_{i=m, h} \eta_{C}^{i} n^{i}}{\bar{L}_{u n} \sum_{i=m, h} \hat{\eta}_{C}^{i} n^{i}}\right)^{\alpha\left(1-\beta_{K, H}\right)}<\left(1-\gamma^{h}\right) .
\end{aligned}
$$

Then, there is an equilibrium in which land prices are given by $p_{L, j}=p_{L_{p}}$ for $j \in J_{p}$ and $p_{L, j}=p_{L_{u n}}$ for $j \in J_{\text {un }}$, with

$$
\begin{aligned}
p_{L_{u n}} & =\alpha\left(1-\beta_{K, H}\right) \beta_{U, C} \bar{p}_{C} Q_{C}\left(\bar{L}_{u n}\right)^{-1} \sum_{i=m, h} \eta_{C}^{i} n^{i}, \\
p_{L_{p}} & =\alpha\left(1-\beta_{K, H}\right) \beta_{U, C} \bar{p}_{C} Q_{C}\left(\bar{L}_{p}\right)^{-1} \sum_{i=m, h} \hat{\eta}_{C}^{i} n^{i},
\end{aligned}
$$

where $Q_{C}=\left(\sum_{i=m, h} N^{i} \bar{e}^{i}\right)^{\beta_{U, C}}\left(N^{s} \bar{e}^{s}\right)^{1-\beta_{U, C}}$. Moreover, in this equilibrium:

1. Agents in group $l$ are criminals who operate and reside in $J_{\text {un }}$. The income of an agent in $l$ is $y^{l}=\delta \beta_{U, C} \bar{p}_{C} Q_{C}\left(N^{l}\right)^{-1} \sum_{i=m, h} \gamma^{i} n^{i}$.

2. Agents in groups $m$ and $h$ are workers and reside in $J_{\text {un }}$. The income of an agent in group $i=h, m$ is $y^{i}=\beta_{U, C} \bar{p}_{C} Q_{C}\left(N^{i}\right)^{-1}\left(1-\gamma^{i}\right) n^{i}$.

3. Agents in group s are workers and reside in $J_{p}$. The income of an agent in group $s$ is $y^{s}=$ $\beta_{U, C} \bar{p}_{C} Q_{C}\left(N^{s}\right)^{-1} \sum_{i=m, h} \hat{\eta}_{C}^{i} n^{i}$. Proof: See Online Appendix.

Lemma 1 shows that, under proper conditions, concentrated protection leads to an extremely segregated city. Neighborhoods in $J_{p}$ are completely safe and have high income per capita and high land and 
housing prices. In the rest of the city, that is, neighborhoods in $J_{u n}$, there is crime, income per capita is low, and land and housing prices are also low. Two mechanisms contribute to produce this equilibrium. First, the payoff from criminal activities does not depend on an agent's productivity, while the payoff from working does. This pushes poor agents into criminal activities. Equation (3) assures that only unskilled agents with the lowest level of labor endowment become criminals. Second, the wealthier an agent, the more harmful criminal activities are for him or her and, as a consequence, the more he or she is willing to sacrifice in order to avoid high-crime areas. This produces a concentration of rich agents in the safe protected neighborhoods and a concentration of poor agents in the unprotected neighborhoods. If the wage-income share of skilled labor is high enough (i.e., $\beta_{U, C}$ is low), these residential decisions lead to higher housing and land prices in the protected neighborhoods than in the unprotected ones. This is consistent with ample causal evidence that crime and insecurity drive housing prices down (Ajzenman, Galiani, and Seira, 2015; Besley and Mueller, 2012; and Linden and Rockoff, 2008). Equation (4) assures that this difference is neither too high, so skilled workers prefer to relocate to the unprotected neighborhoods, nor too low, so unskilled agents prefer to relocate to the protected neighborhoods.

Employing Lemma 1 we can compute the equilibrium utility of an agent in group $i$ :

$$
v_{C}^{i}=\left(N^{i}\right)^{-1} v\left(Q_{C}\right)^{1-\alpha\left(1-\beta_{K, H}\right)}\left(\bar{L}_{r^{i}}\right)^{\alpha\left(1-\beta_{K, H}\right)} \Gamma_{C}^{i},
$$

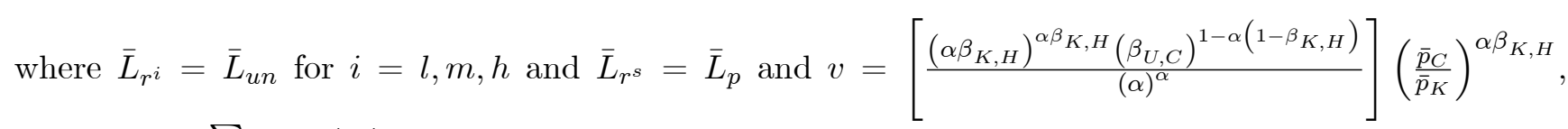
$\Gamma_{C}^{l}=\frac{\delta \sum_{i=m, h} \gamma_{C}^{i} n^{i}}{\left[\sum_{i=m, h} \eta_{C}^{i} n^{i}\right]^{\alpha\left(1-\beta_{K, H}\right)}}, \Gamma_{C}^{m}=\frac{\left(1-\gamma_{C}^{m}\right) n^{m}}{\left[\sum_{i=m, h} \eta_{C}^{i} n^{i}\right]^{\alpha\left(1-\beta_{K, H}\right)}}, \Gamma_{C}^{h}=\frac{\left(1-\gamma_{C}^{h}\right) n^{h}}{\left[\sum_{i=m, h} \eta_{C}^{i} n^{i}\right]^{\alpha\left(1-\beta_{K, H}\right)}}$, and $\Gamma_{C}^{s}=$ $\left[\sum_{i=m, h} \hat{\eta}_{C}^{i} n^{i}\right]^{1-\alpha\left(1-\beta_{K, H}\right)} \cdot v_{C}^{i}$ is affected by international prices, factor endowments, crime, and protection technology. First, there are two common terms, $v$ and $\left(Q_{C}\right)^{1-\alpha\left(1-\beta_{K, H}\right)} \cdot v$ captures a terms of trade effect, where $\bar{p}_{C} / \bar{p}_{K}$ are the terms-of-trade of the city. ${ }^{4}$ Second, there are two terms specific to each group $\left(\bar{L}_{r^{i}}\right)^{\alpha\left(1-\beta_{K, H}\right)}$ and $\Gamma_{C}^{i} . \bar{L}_{r^{i}}$ is the land endowment of the neighborhoods where group $i$ resides. The higher $\bar{L}_{r^{i}}$, the lower the housing prices paid by group $i$. $\Gamma_{C}^{i}$ captures the effect of crime on agents in group $i$. $\Gamma_{C}^{l}$ is increasing in $\gamma^{m}$ and $\gamma^{h}$. Two effects operate. First, as $\gamma^{i}$ for $i=m, h$ increases, the

\footnotetext{
${ }^{4}$ Since there is only one tradable sector, there is no Stolper-Samuelson distributive issue. A higher $\bar{p}_{C} / \bar{p}_{K}$ makes everyone in the city better off. Income is higher and capital to build houses is cheaper for all agents. $\left(Q_{C}\right)^{1-\alpha\left(1-\beta_{K, H}\right)}$ captures the production of tradable goods.
} 
payoff from crime is higher, which increases the income of criminals. Second, this produces a reduction in aggregate income in $J_{u n}$, that is, the neighborhoods where criminals reside, which depresses housing prices in $J_{u n}$. Thus, criminals pay a lower rent. $\Gamma_{C}^{m}$ is decreasing in $\gamma^{m}$, but increasing in $\gamma^{h}$. As $\gamma^{m}$ increases, criminals extract more from agents in group $m$. The reduction in aggregate income depresses housing prices and, hence, agents in group $m$ pay a lower rent. This second effect only partially compensates agents in group $m$ for the reduction in income. As $\gamma^{h}$ increases, criminals extract more from agents in group $h$. This does not affect the income of agents in group $m$. The reduction in aggregate income still depresses housing prices, which benefits agents in group $m$. For analogous reasons, $\Gamma_{C}^{h}$ is decreasing in $\gamma^{h}$, but increasing in $\gamma^{m}$. Finally, $\Gamma_{C}^{s}$ is decreasing in $\gamma^{m}$ and $\gamma^{h}$. Although agents in group $s$ are fully protected in $J_{p}$, they own all the land. As there is more crime in $J_{u n}$, there is a reduction in aggregate income in $J_{u n}$, which induces a decrease in the price of land. This reduces the income of agents in group $s$.

\subsection{Dispersed Protection}

Suppose that protection is evenly dispersed in all neighborhoods. Then, every agent in group $i$ will be able to retain a fraction $\gamma_{D}^{i}=(1-p) \gamma^{i}$ of his or her income, regardless of residential choice. Under dispersed protection, residential choices are simple, because there is no essential difference between neighborhoods. Since protection and, hence, crime, is evenly distributed, only housing prices play a role in residential choices. As a consequence, agents move from one neighborhood to another until there is housing price equalization.

Regarding occupational choices, the same logic we described for concentrated protection also applies here. Specifically, the poorer an agent is, the more incentives he or she has to become a criminal. In equilibrium, only agents in group $l$ choose to be criminals when $\left(1-\gamma_{D}^{l}\right) w^{u} \bar{e}^{l}<y^{l}\left(b_{j}\right)<\left(1-\gamma_{D}^{m}\right) w^{u} \bar{e}^{m}$, where $y^{l}\left(b_{j}\right)=w^{u} \delta\left(N^{l}\right)^{-1} \sum_{i=m, h} \tilde{\gamma}_{D}^{i} \bar{e}^{i} N^{i}$ for $j \in J$ is the income of a criminal when only agents in group $l$ are criminals and $\tilde{\gamma}_{D}^{i}=\frac{\gamma_{D}^{s}+\beta_{U, C}\left[1-\alpha\left(1-\beta_{K, H}\right)\right]\left(\gamma_{D}^{i}-\gamma_{D}^{s}\right)}{\beta_{U, C}\left[1-\alpha\left(1-\beta_{K, H}\right) \eta_{D}^{s}\right]}$.

Lemma 2 formally characterizes the equilibrium under dispersed protection. Let $\eta_{D}^{i}=1-\gamma_{D}^{i}+\delta \gamma_{D}^{i}$, $\tilde{\eta}_{D}^{i}=\frac{\beta_{U, C} \eta_{D}^{i}+\left(1-\beta_{U, C}\right) \eta_{D}^{s}}{\beta_{U, C}\left[1-\alpha\left(1-\beta_{K, H}\right) \eta_{D}^{s}\right]}$ and $\hat{\eta}_{D}^{i}=\frac{\alpha\left(1-\beta_{K, H}\right) \beta_{U, C} \eta_{D}^{i}+\left(1-\beta_{U, C}\right)}{\beta_{U, C}\left[1-\alpha\left(1-\beta_{K, H}\right) \eta_{D}^{s}\right]}$.

Lemma 2 Dispersed Protection. Suppose that protection is evenly distributed in all neighborhoods, 
and Assumption 1 and the following condition hold:

$$
\left(1-\gamma_{D}^{l}\right) \bar{e}^{l}<\delta\left(N^{l}\right)^{-1} \sum_{i=m, h} \tilde{\gamma}_{D}^{i} \bar{e}^{i} N^{i}<\left(1-\gamma_{D}^{m}\right) \bar{e}^{m}
$$

Then, there is an equilibrium in which land prices are the same in all neighborhoods and given by

$$
p_{L}=\alpha\left(1-\beta_{K, H}\right) \beta_{U, C} \bar{p}_{C} Q_{C}(\bar{L})^{-1} \sum_{i=h, m} \tilde{\eta}_{D}^{i} n^{i},
$$

where $Q_{C}=\left(\sum_{i=m, h} N^{i} \bar{e}^{i}\right)^{\beta_{U, C}}\left(N^{s} \bar{e}^{s}\right)^{1-\beta_{U, C}}$. Moreover, in this equilibrium:

1. Agents in group $l$ are criminals and operate in all neighborhoods. The income of an agent in group $l$ is $y^{l}=\delta \beta_{U, C} \bar{p}_{C} Q_{C}\left(N^{l}\right)^{-1} \sum_{i=m, h} \tilde{\gamma}_{D}^{i} n^{i}$.

2. Agents in group $i=m, h, s$ are workers. The income of an agent in group $i=h, m$ is $y^{i}=\beta_{U, C} \bar{p}_{C} Q_{C}\left(N^{i}\right)^{-1}\left(1-\gamma_{D}^{i}\right) n^{i}$, while the income of an agent in group $s$ is $y^{s}=$ $\beta_{U, C} \bar{p}_{C} Q_{C}\left(N^{s}\right)^{-1}\left(1-\gamma_{D}^{s}\right) \sum_{i=h, m} \hat{\eta}_{D}^{i} n^{i}$. Proof: See Online Appendix.

Lemma 2 shows that evenly dispersed protection leads to an integrated city. The mechanism behind this result is simple. When protection is the same in all neighborhoods, free mobility of workers and criminals induces equalization of income per capita and housing prices among neighborhoods. If one neighborhood has a higher income, criminals move there and workers leave the neighborhood until the difference disappears.

Employing Lemma 2 we can compute the equilibrium utility of an agent in group $i$ :

$$
v_{D}^{i}=\left(N^{i}\right)^{-1} v\left(Q_{C}\right)^{1-\alpha\left(1-\beta_{K, H}\right)}(\bar{L})^{\alpha\left(1-\beta_{K, H}\right)} \Gamma_{D}^{i},
$$

where $\Gamma_{D}^{l}=\frac{\delta \sum_{i=m, h} \tilde{\gamma}_{D}^{i} n^{i}}{\left[\sum_{i=h, m} \tilde{\eta}_{D}^{i} n^{i}\right]^{\alpha\left(1-\beta_{K, H}\right)}}, \Gamma_{D}^{m}=\frac{\left(1-\gamma_{D}^{m}\right) n^{m}}{\left[\sum_{i=h, m} \tilde{\eta}_{D}^{i} n^{i}\right]^{\alpha\left(1-\beta_{K, H}\right)}}, \Gamma_{D}^{h}=\frac{\left(1-\gamma_{D}^{h}\right) n^{h}}{\left[\sum_{i=h, m} \tilde{\eta}_{D}^{i} n^{i}\right]^{\alpha\left(1-\beta_{K, H}\right)}}$ and $\Gamma_{D}^{s}=\frac{\left(1-\gamma_{D}^{s}\right) \sum_{i=h, m} \hat{\eta}_{D}^{i} n^{i}}{\left[\sum_{i=h, m} \tilde{\eta}_{D}^{i} n^{i}\right]^{\alpha\left(1-\beta_{K, H}\right)}} \cdot v_{D}^{i}$ is affected by international prices, factor endowments, crime, and protection technology. First, there are three common terms: $v,\left(Q_{C}\right)^{1-\alpha\left(1-\beta_{K, H}\right)}$, and $(\bar{L})^{\alpha\left(1-\beta_{K, H}\right)} .^{5}$ Second, there is a term specific to each group $\Gamma_{D}^{i}$. $\Gamma_{D}^{l}$ is increasing in $\gamma_{D}^{m}, \gamma_{D}^{h}$, and $\gamma_{D}^{s}$. $\Gamma_{D}^{m}$ is decreasing in $\gamma_{D}^{m}$ and increasing in $\gamma_{D}^{h}$ and $\gamma_{D}^{s}$. $\Gamma_{D}^{h}$ is decreasing in $\gamma_{D}^{h}$ and increasing in $\gamma_{D}^{m}$ and $\gamma_{D}^{s}$.

\footnotetext{
${ }^{5} v$ is a terms-of-trade effect. The higher the terms of trade, the better off everyone in the city is. $Q_{C}$ captures the production of tradable goods, while $\bar{L}$ is the total endowment of land in the city. The higher the $\left(Q_{C}\right)^{1-\alpha\left(1-\beta_{K, H}\right)}$, the higher the income of all agents. The higher the $\bar{L}$, the lower the housing prices.
} 


\section{Regime Comparisons}

This section compares several key variables under concentrated and dispersed protection. First, we study how the protection regime affects crime, aggregate income, and housing prices. Second, we consider the utility of each group as well as aggregate welfare.

\subsection{Crime}

The model supports at least two different measures of crime. First, we can focus on the number and characteristics of the criminals. Second, we can compute the value of stolen goods, that is., total plunder. Under the assumption and conditions in Lemmas 1 and 2, regardless of the protection regime, only agents in group $l$ choose to become criminals. Thus, the protection regime does not change either occupational choices or the number and characteristics of the criminals. On the contrary, the protection regime affects the value of stolen goods. Indeed, the value of stolen goods is higher under dispersed protection if and only if $\sum_{i=m, h}\left(\gamma^{i}-\tilde{\gamma}_{D}^{i}\right) N^{i} \bar{e}^{i}<0$ or, which is equivalent, $\sum_{i=m, h}\left[p \gamma^{i}-(1-p) \gamma^{s} \hat{\eta}_{D}^{i}\right] n^{i}<0$. The intuition behind this inequality is straightforward. Under concentrated protection, criminals extract more from agents in groups $m$ and $h\left(p \gamma^{i}\right)$, but agents in group $s$ are fully protected. Under dispersed protection, criminals also extract from agents in group $s\left((1-p) \gamma^{s} \hat{\eta}_{D}^{i}>0\right)$. It is interesting to study when it is more likely that this inequality holds. After some simple algebra we can express it as follows:

$$
\sum_{i=m, h} \gamma^{i} n^{i}<\frac{\left[\frac{1-\beta_{U, C}}{\beta_{U, C}}+\alpha\left(1-\beta_{K, H}\right)\right](1-p) \gamma^{s}}{p\left[1-\alpha\left(1-\beta_{K, H}\right)\right]+(1-p) \alpha\left(1-\beta_{K, H}\right) \gamma^{s}(1-\delta)}
$$

Thus, crime, measured as the value of stolen goods, is more likely to be higher under dispersed than concentrated protection when the wage-income share of skilled labor is higher $\left(\beta_{U, C} \text { is lower }\right)^{6}$, when the reduction in the effectiveness of protection when the police are dispersed is lower ( $p$ is lower), when criminals can extract more from skilled workers $\left(\gamma^{s}\right.$ is higher $)$, and when criminals can extract less from unskilled workers $\left(\gamma^{i}\right.$ is lower for $\left.i=h, m\right)$.

\footnotetext{
${ }^{6}$ See Kang (2016) for empirical evidence consistent with this result.
} 


\subsection{Aggregate Income}

The protection regime also affects aggregate income, which is higher under concentrated protection if and only if $\sum_{i=m, h}\left(\tilde{\eta}_{C}^{i}-\tilde{\eta}_{D}^{i}\right) n^{i}>0$, where $\tilde{\eta}_{C}^{i}=\frac{\beta_{U, C} \eta_{C}^{i}+\left(1-\beta_{U, C}\right)}{\beta_{U, C}\left[1-\alpha\left(1-\beta_{K, H}\right)\right]}=\hat{\eta}_{C}^{i}+\eta_{C}^{i}$ and $\tilde{\eta}_{D}^{i}=\frac{\beta_{U, C} \eta_{D}^{i}+\left(1-\beta_{U, C}\right) \eta_{D}^{s}}{\beta_{U, C}\left[1-\alpha\left(1-\beta_{K, H}\right) \eta_{D}^{s}\right]}$. It is interesting to study when it is more likely that this inequality holds. After some simple algebra we can express it as follows:

$$
\beta_{U, C}<\frac{\gamma^{s}}{\left[1-\alpha\left(1-\beta_{K, H}\right)\right] \gamma^{s}+\left[\frac{p}{1-p}+\alpha\left(1-\beta_{K, H}\right)(1-\delta) \gamma^{s}\right] \sum_{i=m, h} \gamma^{i} n^{i}} .
$$

Thus, aggregate income is more likely to be higher under concentrated protection when the wage-income share of skilled labor is higher $\left(\beta_{U, C}\right.$ is lower), when the reduction in the effectiveness of protection after the police are dispersed is lower ( $p$ is lower), when criminals can extract more from skilled workers $\left(\gamma^{s}\right.$ is higher), and when criminals can extract less from unskilled workers $\left(\gamma^{i}\right.$ is lower for $\left.i=h, m\right)$.

\subsection{Housing Prices}

Housing and land prices are also affected by the protection regime. In particular, $p_{L_{u n}}<p_{L}<$ $p_{L_{p}}$ if and only if $\left(\bar{L}_{u n}\right)^{-1} \sum_{i=m, h} \eta_{C}^{i} n^{i}<(\bar{L})^{-1} \sum_{i=m, h} \tilde{\eta}_{D}^{i} n^{i}<\left(\bar{L}_{p}\right)^{-1} \sum_{i=m, h} \hat{\eta}_{C}^{i} n^{i}$, where $\tilde{\eta}_{D}^{i}=$ $\frac{\beta_{U, C} \eta_{D}^{i}+\left(1-\beta_{U, C}\right) \eta_{D}^{s}}{\beta_{U, C}\left[1-\alpha\left(1-\beta_{K, H}\right) \eta_{D}^{s}\right]}$ and $\hat{\eta}_{C}^{i}=\frac{\beta_{U, C} \alpha\left(1-\beta_{K, H}\right) \eta_{C}^{i}+\left(1-\beta_{U, C}\right)}{\beta_{U, C}\left[1-\alpha\left(1-\beta_{K, H}\right)\right]}$. After some simple algebra we can express it as follows:

$$
\frac{\bar{L}_{u n}}{\bar{L}}>\max \left\{\frac{\sum_{i=m, h} \eta_{C}^{i} n^{i}}{\sum_{i=m, h} \tilde{\eta}_{D}^{i} n^{i}}, \frac{\sum_{i=m, h}\left(\tilde{\eta}_{D}^{i}-\hat{\eta}_{C}^{i}\right) n^{i}}{\sum_{i=m, h} \tilde{\eta}_{D}^{i} n^{i}}\right\} .
$$

Thus, if equation (13) holds, a move from concentrated to dispersed protection will increase housing prices in $J_{u n}$ and reduce housing prices in $J_{p}$. Moreover, this condition is more likely to be satisfied the higher the wage-income share of skilled labor $\left(\beta_{U, C}\right.$ is lower).

The following proposition summarizes the results.

Proposition 1 Under the assumptions and conditions in Lemmas 1 and 2.

1. Crime is lower under concentrated protection if and only if equation (11) holds. Moreover, equation (11) is more likely to be satisfied the higher the wage-income share of skilled labor $\left(\beta_{U, C}\right.$ is lower), the lower $p$, the more criminals can extract from skilled workers ( $\gamma^{s}$ is higher), and the less criminals can extract from unskilled workers ( $\gamma^{i}$ is lower for $\left.i=h, m\right)$. 
2. Aggregate income is higher under concentrated protection if and only if equation (12) holds. Moreover, equation (12) is more likely to be satisfied the higher wage-income share of skilled labor $\left(\beta_{U, C}\right.$ is lower), the lower $p$, the more criminals can extract from skilled workers ( $\gamma^{s}$ is higher) and the less criminals can extract from unskilled workers ( $\gamma^{i}$ is lower for $\left.i=h, m\right)$.

3. Housing prices under dispersed protection are between housing prices in unprotected neighborhoods and protected neighborhoods under concentrated protection $\left(p_{L_{u n}}<p_{L}<p_{L_{p}}\right)$ if and only if equation (13) holds. Moreover, equation (13)is more likely to be satisfied the higher the wage-income share of skilled labor $\left(\beta_{U, C}\right.$ is lower $)$. Proof: See Online Appendix.

The intuition behind Proposition 1 is as follows: Under concentrated protection, skilled agents are fully protected and the rest of the agents are not protected at all. Housing is expensive for those protected and cheap for those not protected. Under dispersed protection, all groups get the same level of protection. This means that previously protected agents (i.e., skilled workers) now get less protection and previously unprotected workers (i.e., unskilled workers) now receive more protection. The effects on crime and aggregate income are ambiguous because under dispersed protection, criminals can extract less from unskilled workers, but they can also rob skilled workers. Income inequality and, in particular, the wage-income share of skilled labor, plays a key role. When the wage-income share of skilled labor is high, it is more likely that crime is lower and income is higher under concentrated protection. In other words, dispersing the police is likely to produce an increase in crime and a reduction in aggregate income in an unequal society, and the opposite effects in a more equalitarian one. Housing prices are also affected by the protection regime. Dispersing the police will induce convergence in the housing prices in both neighborhoods. Thus, housing prices will decrease for previously protected workers and increase for previously unprotected workers.

\subsection{Welfare}

From a normative point of view we can study which protection regime is better. First, we explore the distributive effects associated with a change in the protection regime. Second, we compare aggregate welfare under both regimes. As a benchmark we assume a utilitarian welfare function. Formally, aggregate 
welfare is given by $W=\sum_{j=1,2} \sum_{i=l, m, h, s} v^{i}\left(o^{i}, j\right) N^{i}\left(o^{i}, j\right)$. The following proposition summarizes welfare comparisons.

Proposition 2 Welfare. Under the assumptions and conditions in Lemmas 1 and 2. Then:

1. Socioeconomic Groups. (a) Group l. $v_{D}^{l}>v_{C}^{l}$ if and only if $\frac{\bar{L}_{u n}}{\bar{L}}<\left(\frac{\Gamma_{D}^{l}}{\Gamma_{C}^{l}}\right)^{\frac{1}{\alpha\left(1-\beta_{K, H}\right)}}$.

Groups $m$ and $h . v_{D}^{i}>v_{C}^{i}$ for $i=m, h$ if and only if $\frac{\bar{L}_{\overline{u n}}}{\bar{L}}<\left(\frac{\Gamma_{D}^{i}}{\Gamma_{C}^{i}}\right)^{\frac{1}{\alpha\left(1-\beta_{K, H}\right)}}$. (c) Group $s$. $v_{C}^{s}>v_{D}^{s}$ if and only if $\frac{\bar{L}_{u n}}{L}<1-\left(\frac{\Gamma_{D}^{s}}{\Gamma_{C}^{s}}\right)^{\frac{1}{\alpha\left(1-\beta_{K, H}\right)}}$.

2. Unskilled versus skilled agents. Let $W_{D}^{u}=\sum_{i=l, m, h} N^{i} v_{D}^{i}$ and $W_{C}^{u}=\sum_{i=l, m, h} N^{i} v_{C}^{i}$. Then, $W_{D}^{u}>W_{C}^{u}$ and, hence, at least for one $i=l, m, h$ it must be the case that $v_{D}^{i}>v_{C}^{i}$.

3. Aggregate welfare. Let $W_{D}=\sum_{i=l, m, h, s} N^{i} v_{D}^{i}$ and $W_{C}=\sum_{i=l, m, h, s} N^{i} v_{C}^{i}$. Then, $W_{C}>W_{D}$ if and only if

$$
\Gamma_{C}^{u}\left(\frac{\bar{L}_{u n}}{\bar{L}}\right)^{\alpha\left(1-\beta_{K, H}\right)}+\Gamma_{C}^{s}\left(\frac{\bar{L}_{p}}{\bar{L}}\right)^{\alpha\left(1-\beta_{K, H}\right)}>\Gamma_{D}
$$

where $\Gamma_{C}^{u}=\sum_{i=l, m, h} \Gamma_{C}^{i}$ and $\Gamma_{D}=\sum_{i \in I} \Gamma_{D}^{i}$. Moreover, if $\delta \in\left(\delta_{L}, \delta_{H}\right)$ and $\frac{\bar{L}_{u n}}{L_{p}} \in\left(\lambda_{L}, \lambda_{H}\right)$, there exist $\bar{\beta} \in(0,1)$ and $\hat{\beta}^{\min } \in(0,1)$ such that $W_{C}<W_{D}$ for $\frac{1-\beta_{U, C}}{\beta_{U, C}} \in\left(0, \min \left\{\frac{1-\bar{\beta}}{\bar{\beta}}, \frac{1-\hat{\beta}^{\min }}{\hat{\beta}^{\min }}\right\}\right)$ and $W_{C}>W_{D}$ for $\frac{1-\beta_{U, C}}{\beta_{U, C}} \in\left(\min \left\{\frac{1-\bar{\beta}}{\bar{\beta}}, \frac{1-\hat{\beta}^{\min }}{\hat{\beta}^{\min }}\right\}, \frac{1-\hat{\beta}^{\min }}{\hat{\beta}^{\min }}\right)$. Proof: See Online Appendix.

Part 1 of Proposition 2 confirms that a change in the protection regime will tend to generate winners and losers. Under concentrated protection, skilled agents enjoy full protection and unskilled agents are completely unprotected. Under dispersed protection, all agents enjoy the same intermediate level of protection. The changes in the protection levels affect the income of each group. Criminals can extract more from unskilled workers under concentrated protection, but they cannot target skilled workers. Unskilled workers are better protected under dispersed protection, while skilled workers are better protected under concentrated protection. The changes in the protection levels also affect housing prices. For example, if equation (13) holds, criminals and unskilled workers pay higher housing prices under dispersed protection, while skilled workers pay higher housing prices under concentrated protection. Moreover, the variation in the income of a group responds to the change in the protection level, while the variation in housing prices responds to the change in aggregate income. Therefore, for one group the effect on income may dominate the effect on housing prices, while for another group the opposite happens. 
Part 2 of Proposition 2 puts some order to the type of distributive effects induced by a change in the protection regime. At least one group of unskilled agents is better off under dispersed than under concentrated protection. This, however, does not necessarily mean that all unskilled agents unanimously prefer dispersed protection. The reason is that dispersing the police could produce a significant increase in the housing prices paid by unskilled workers, which dominate the increase in their incomes. Part 2 of Proposition 2 also implies that preferences for concentrated and dispersed protection may not be monotonic with respect to income. This suggests that there is room for atypical political coalitions made up of poor and rich agents. Figure 1 illustrates this point for different values of $p$ (Figure 1.a) and $\beta$ (Figure 1.b). For example, if $p=0.50$ and $\beta_{U, C}=0.50$, then $v_{C}^{l}=0.36<v_{D}^{l}=0.63, v_{C}^{m}=0.93>v_{D}^{m}=0.85$, $v_{C}^{h}=1.36<v_{D}^{h}=1.64$, and $v_{C}^{s}=10.39>v_{D}^{s}=8.75$. Thus, in this case, groups $m$ and $s$ will support concentrated protection, while groups $l$ and $h$ will prefer dispersed protection.

Figure 1: $v_{C}^{i} / v_{D}^{i}$ and $W_{C} / W_{D}^{7}$

a. $v_{C}^{i} / v_{D}^{i}$ as a function of $p$

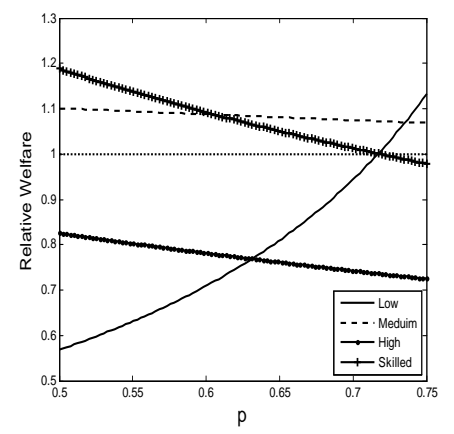

b. $v_{C}^{i} / v_{D}^{i}$ as a function of $\beta_{U, C}$

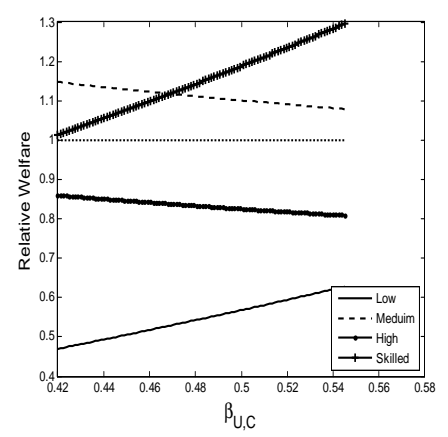

c. $W_{C} / W_{D}$ as a function of $\beta_{U, C}$

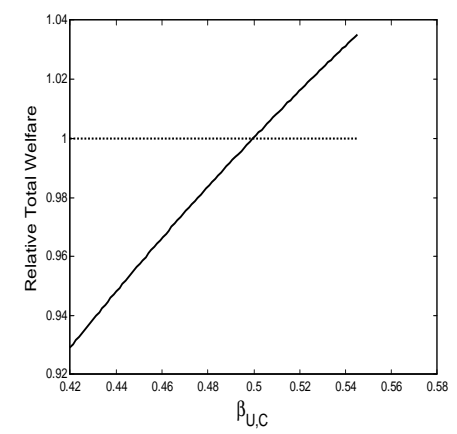

Part 3 of Proposition 2 shows that aggregate welfare is higher under concentrated protection for an unequal society, while the opposite is true for an equalitarian one. Figure 1.c illustrates this point. The intuition behind this result is simple. When skilled agents represent an important share of aggregate income, aggregate welfare is higher when they are well protected. This suggests that unequal societies could face a difficult dilemma. The welfare-maximizing protection regime (i.e., concentrated protection) makes

\footnotetext{
${ }^{7}$ For the numerical example we consider the following values for the parameters of the model: (1) population shares: $N^{l}=0.35, N^{m}=0.5, N^{h}=0.1$, and $N^{s}=0.05 ;(2)$ labor endowments: $e^{l}=1, e^{m}=3, e^{h}=7$, and $e^{s}=10 ;(3)$ preferences: $\alpha=0.5$; (4) production functions: $\beta_{U, C}=0.5$ (for Figure 1.a), and $\beta_{K, H}=0.5$; (5) prices of tradable goods: $\bar{p}_{C}=1$, and $\bar{p}_{K}=2 ;(6)$ Crime: $\gamma^{l}=0.1, \gamma^{m}=0.2, \gamma^{h}=0.5, \gamma^{s}=0.7$, and $\delta=0.5$; and (7) protection technology: $\bar{L}_{u n}=100$, and $\bar{L}_{p}=10$ and $p=0.5$ (for Figures 1.b and 1.c).
} 
society even more unequal. On the other hand, in more equalitarian societies, the welfare-maximizing protection regime (i.e., dispersed protection) will reduce social disparities.

\subsection{Compensations}

A solution to these problematic distributive effects is to provide proper compensation. Suppose that concentrated protection maximizes aggregate welfare, but the government is particularly concerned about the impact of this regime on poor agents. Indeed, from Part 2 of Proposition 2 we know that concentrated protection induces a lower payoff than dispersed protection for unskilled agents. However, the government can compensate the losers by paying subsidies financed with taxes on the winners. Let $\bar{p}_{C} \tau_{f}^{i}$ be the transfer received/paid by each agent in group $i$. Then, the government budget constraint is:

$$
\sum_{i \in I} \tau_{f}^{i} N^{i}=0
$$

In the Online Appendix we characterize the equilibrium under concentrated protection when the government provides compensation. Equations (3) and (4), as well as equations (5) and (6) for equilibrium land prices, must be modified, but the segregated city equilibrium persists. We also compute the transfers that the government must provide in order to make each unskilled agent equally well-off between concentrated and dispersed protection. Formally, $\left(\tau_{f}^{l}, \tau_{f}^{m}, \tau_{f}^{h}\right)$ is the solution to the following system of equations:

$$
\begin{gathered}
{\left[\frac{\sum_{i=m, h} \gamma^{i} n^{i}}{\sum_{i=m, h} \tilde{\gamma}_{D}^{i} n^{i}}+\frac{\delta \sum_{i=m, h} \gamma^{i} \tau_{f}^{i} N^{i}+\tau_{f}^{l} N^{l}}{\delta \beta_{U, C} Q_{C} \sum_{i=m, h} \tilde{\gamma}_{D}^{i} n^{i}}\right]\left[\frac{\Psi\left(\tau_{f}^{l}, \tau_{f}^{m}, \tau_{f}^{h}\right) \bar{L}_{u n}}{\bar{L}}\right]^{\alpha\left(1-\beta_{K, H}\right)}=1,} \\
{\left[\left(\frac{1-\gamma^{m}}{1-\gamma_{D}^{m}}\right)\left(1+\frac{\tau_{f}^{m} N^{m}}{n^{m} Q_{C}}\right)\right]\left[\frac{\Psi\left(\tau_{f}^{l}, \tau_{f}^{m}, \tau_{f}^{h}\right) \bar{L}_{u n}}{\bar{L}}\right]^{\alpha\left(1-\beta_{K, H}\right)}=1,} \\
{\left[\left(\frac{1-\gamma^{h}}{1-\gamma_{D}^{h}}\right)\left(1+\frac{\tau_{f}^{h} N^{h}}{n^{h} Q_{C}}\right)\right]\left[\frac{\Psi\left(\tau_{f}^{l}, \tau_{f}^{m}, \tau_{f}^{h}\right) \bar{L}_{u n}}{\bar{L}}\right]^{\alpha\left(1-\beta_{K, H}\right)}=1,}
\end{gathered}
$$

where $\Psi\left(\tau_{f}^{l}, \tau_{f}^{m}, \tau_{f}^{h}\right)=\frac{\beta_{U, C} Q_{C} \sum_{i=h, m} \tilde{\eta}_{D}^{i} n^{i}}{\beta_{U, C} Q_{C} \sum_{i=m, h} \eta_{C}^{i} n^{i}+\sum_{i=m, h} \eta_{C}^{i} \tau_{f}^{i} N^{i}+\tau_{f}^{l} N^{l}}$.

There are several interesting features about equations (16)-(18). First, with no transfers, unskilled agents are better off under dispersed than concentrated protection (Part 2 of Proposition 2). Therefore, 
it must be the case that $\sum_{i=l, m, h} \tau_{f}^{i} N^{i}>0$, which means that skilled agents must be taxed. In fact, from the government budget constraint in equation (15), we have $\tau_{f}^{s}=-\left(N^{s}\right)^{-1} \sum_{i=l, m, h} \tau_{f}^{i} N^{i}<0$. Second, compensation must take into account the impact of the protection regime on incomes (first squared bracket on the left-hand side of each equation) and housing prices (second squared bracket on the left-hand side of each equation). Moreover, while the effects on housing prices are the same for all socioeconomic groups, the effects on incomes differ. Finally, note that compensations are interrelated and cannot be computed separately. The reason is that general equilibrium effects matter. As one group receives a higher transfer, the aggregate income of unprotected neighborhoods increases, which leads to higher housing prices for all unskilled agents (see the denominator of $\Psi\left(\tau_{f}^{l}, \tau_{f}^{m}, \tau_{f}^{h}\right)$ ). A higher transfer for groups $m$ and $h$ also affects the payoff for criminals (see the first squared bracket in equation (16)).

\section{Private Protection}

Previous empirical work has shown that when crime levels are high, rich agents buy private security to protect themselves. For example, Di Tella, Galiani, and Schargrodsky (2010) and Levitt (1999) show that the rich, unlike the poor, are able to protect their homes by hiring security services and/or installing security devices. This enables them to avoid being robbed as much as they would be if they were not be able to self-protect. This section extends the model to include private protection. The main motivation is to explore the robustness of the segregated and integrated city equilibria when agents have access to private security. We treat private protection as a club good complement with public protection in the sense that it requires public protection to be effective.

Consider the model in Section 2, but assume that there is a technology to build protected areas. In particular, suppose that developers can combine $l$ units of adjacent land in neighborhood $j$ with $k(l) / A\left(p_{j}\right)$ units of capital and obtain $l$ units of fully protected land, where $A(0)=0$ and $A\left(p_{j}\right)$ is increasing in $p_{j}$. Whoever resides in a house built on protected land is not affected by criminals. The average cost of developing $l$ units of fully protected land in neighborhood $j$ is $C(l)=\frac{p_{K} k(l)}{A\left(p_{j}\right) l}+p_{L, j}$, where $p_{L, j}$ is the price of land without any security investments. Assume that $k(l) / l$ has a minimum at $l_{\min }>0$, and let $c_{\min }=k\left(l_{\min }\right) / l_{\min }$. Then, if there is free entry, developers will keep converting land into protected areas until, in equilibrium, the price of a unit of land in a fully protected area is 
$p_{L_{p}, j}=\frac{p_{K} c_{\min }}{A\left(p_{j}\right)}+p_{L, j}$. Moreover, each of these developments will contain $l_{\min }$ units of land. ${ }^{8}$ Also note that, since $A\left(p_{j}\right)$ is increasing in $p_{j}, p_{L_{p}, j}$ is decreasing in $p_{j}$, that is, developing a fully protected unit of land is cheaper in a neighborhood with higher public protection. In other words, private and public protection are complements.

It is simple to prove that private security will not emerge when public protection is concentrated. If a neighborhood is fully protected by the police, no agent will be willing to pay higher housing prices to cover the extra costs of private protection because there is no gain from extra protection. If a neighborhood is not protected by the police, the cost of private security is prohibitive. Thus, when police protection is concentrated, the segregated city equilibrium characterized in Lemma 1 persists even when agents have access to private security.

On the contrary, when police protection is evenly deployed across the city, private protection can affect the integrated equilibrium described in Lemma 2. As the following lemma formally shows, under proper conditions, skilled agents prefer to reside in fully protected areas with private and public protection, while unskilled agents are not willing to pay the high housing prices in those areas. Thus, private security may restore a segregated city equilibrium in which rich agents reside in safe areas, while crime is concentrated in poor neighborhoods.

Lemma 3 Private Protection. Suppose that agents have access to private protection.

1. If police protection is concentrated, private protection has no effect on the equilibrium, that is, Lemma 1 applies.

2. Suppose that police protection is evenly distributed in all neighborhoods and Assumption 1 holds.

\footnotetext{
${ }^{8}$ If we assume an integer number of symmetric developers, free entry will not necesarilly lead to zero profits. A simple way to solve this problem is to assume that one developer has a cost curve with a flat range from $l_{\min }$ to $l_{\min }+1$. Under free entry, this developer will convert $l \in\left[l_{\min }, l_{\min }+1\right]$ units of land into protected areas at a unit $\operatorname{cost}$ of $\frac{p_{K} c_{\min }}{A\left(p_{j}\right)}+p_{L, j}$. If the zero profit condition induces a non-integer number of symmetric developers, the developer with the flat range in the cost curve will absorb the difference.
} 
Let

$$
\begin{aligned}
\bar{c}^{i} & =\frac{\alpha\left(1-\beta_{K, H}\right) \beta_{U, C} \bar{p}_{C} Q_{C} A(p)}{\bar{p}_{K} \bar{L}}\left[\frac{\sum_{i=m, h} \hat{\eta}_{D}^{i} n^{i}}{\bar{\lambda}^{i}}-\frac{\sum_{i=m, h} \eta_{D}^{i} n^{i}}{1-\bar{\lambda}^{i}}\right] \\
\bar{\lambda}^{i} & =\frac{\left[1-\gamma^{i}(1-p)\right]^{\frac{1}{\alpha\left(1-\beta_{K, H}\right)}} \sum_{i=m, h} \hat{\eta}_{D}^{i} n^{i}}{\sum_{i=m, h} \eta_{D}^{i} n^{i}+\left[1-\gamma^{i}(1-p)\right]^{\frac{1}{\alpha\left(1-\beta_{K, H}\right)}} \sum_{i=m, h} \hat{\eta}_{D}^{i} n^{i}}
\end{aligned}
$$

(a) Suppose that $\bar{c}^{h}<c_{\min }<\bar{c}^{s}$ and $\left[1-\gamma^{l}(1-p)\right] \bar{e}^{l}<\delta\left(N^{l}\right)^{-1} \sum_{i=m, h}(1-p) \gamma^{i} N^{i} \bar{e}^{i}<$ $\left[1-\gamma^{m}(1-p)\right] \bar{e}^{m}$. Then, there is an equilibrium in which the city is segregated. Only agents in group $l$ are criminals; all unskilled agents reside in areas without private security; and agents in group s reside in fully protected areas with private security. The size of fully protected land is $L_{p}=\frac{-b-\sqrt{b^{2}-4 a c}}{2 a}$, where $a=\frac{\bar{p}_{K} c_{\min }}{A(p)}, b=-\alpha\left(1-\beta_{K, H}\right) \beta_{U, C} \bar{p}_{C} Q_{C} \sum_{i=m, h}\left(\hat{\eta}_{D}^{i}+\eta_{D}^{i}\right) n^{i}-a \bar{L}$ and $c=\alpha\left(1-\beta_{K, H}\right) \beta_{U, C} p_{C} Q_{C}\left(\sum_{i=m, h} \hat{\eta}_{D}^{i} n^{i}\right) \bar{L}$. Land prices are given by:

$$
\begin{aligned}
p_{L} & =\alpha\left(1-\beta_{K, H}\right) \beta_{U, C} p_{C} Q_{C}\left(\bar{L}-L_{p}\right)^{-1} \sum_{i=m, h} \eta_{D}^{i} n^{i}, \\
p_{L_{p}} & =\alpha\left(1-\beta_{K, H}\right) \beta_{U, C} p_{C} Q_{C}\left(L_{p}\right)^{-1} \sum_{i=m, h} \hat{\eta}_{D}^{i} n^{i} .
\end{aligned}
$$

(b) Suppose that $c_{\min }>\bar{c}^{s}$. Then private protection has no effect on the equilibrium, that is, Lemma 2 applies. Proof: See Online Appendix.

Lemma 3 suggests that dispersing the police force could not be enough to equalize criminal activities across the city. If $\bar{c}^{h}<c_{\min }<\bar{c}^{s}$, even when public protection is evenly dispersed in the city, skilled agents will use private protection to endogenously isolate themselves in fully protected areas. Unskilled workers will find housing prices in those areas too expensive and, hence, they will reside in areas with cheaper housing, but more crime. Of course, this might not be the case if skilled agents find private protection too expensive. Indeed, when $c_{\min }>\bar{c}^{s}$, agents in group $s$ are not willing to reside in a neighborhood with private protection. Therefore, there is no private protection and the dispersed equilibrium persists.

In Lemma 3, we have focused on a situation where only agents in group $s$ have an incentive to buy private security. This could be easily relaxed. If $c_{\min }<\bar{c}^{h}$, then at least some unskilled agents also prefer to reside in a neighborhood with private protection. As a consequence, the areas with private protection will expand and, provided that there are some unskilled agents who are not willing to buy 
private protection, there will be a segregated equilibrium with safe areas with public and private security populated by rich agents and poor areas with higher crime rates and only protected by public security.

\section{A Continuum of Agent Types and Occupational Choices}

Previous sections have focused on a region of the parameter space in which the protection regime does not change occupational choices. It is possible to extend the analysis of the model with four socioeconomic groups to study regions in which the change in the protection strategy induces agents in group $m$ to modify their occupational choices. However, it is more natural to attack this problem in a model with a continuum of agent types, where any change in the payoffs of legal versus illegal activities induces a change in the marginal agent. Furthermore, it is easier to incorporate some extensions in the model with a continuum of agent types. In particular, we can introduce almost any distribution of labor endowments, allow for more general ownership structures of land, and allow for criminals to distribute their plunder unevenly. Unfortunately, the model with a continuum of agent types has one important disadvantage. Although we can summarize the equilibrium in a few equations, we need numerical solutions to obtain a full characterization of the equilibrium.

Consider a city integrated by a set of neighborhoods $J$, each with a fixed quantity of land $\bar{L}_{j}$ and let $\bar{L}=\sum_{j \in J} \bar{L}_{j}$. The city is a small open economy populated by a continuum of agents. Each agent has labor endowment $e \in\left[e^{L}, e^{H}\right]$, with $0<e^{L}<e^{H}$. The cumulative distribution function of $e$ is $F(e)$, which has a continuous density $f(e)>0$ for all $e$. Some agents also own land. In particular, let $s_{L}(e)$ be the share of $\bar{L}_{j}$ owned by agent $e$. Assume that $s_{L}(e)=0$ for $e<\bar{e}, s_{L}(e)>0$ for $e \geq \bar{e}$, $s_{L}^{\prime}(e) \geq 0$ and $\int_{\bar{e}}^{e^{H}} s_{L}(e) f(e) d e=1$. Labor is employed in the production of a tradable consumption good, whose production function is $Q_{C}=N$ and whose price is exogenously given by $p_{C}=\bar{p}_{C}>0$. Thus, the wage rate is $w=\bar{p}_{C}$. Land and imported capital are used to build houses. The production function is $Q_{H, j}=\left(K_{H, j}\right)^{\beta_{K, H}}\left(\bar{L}_{j}\right)^{1-\beta_{K, H}}$, where $K_{H, j}$ stands for capital used in the construction of houses in neighborhood $j$. The price of capital is exogenously given by $p_{K}=\bar{p}_{K}>0$. Thus, the demand of capital $\left(K_{H, j}\right)$, the price of houses $\left(p_{H, j}\right)$, and the supply of houses $\left(Q_{H, j}\right)$ in each neighborhood are given by $K_{H, j}=p_{L, j} \bar{L}_{j} \beta_{K, H} / \bar{p}_{K}\left(1-\beta_{K, H}\right), p_{H, j}=\left(\bar{p}_{K} / \beta_{K, H}\right)^{\beta_{K, H}}\left(p_{L, j} /\left(1-\beta_{K, H}\right)\right)^{1-\beta_{K, H}}$ and $p_{H, j} Q_{H, j}=p_{L, j} \bar{L}_{j} /\left(1-\beta_{K, H}\right)$, respectively, where $p_{L, j}$ is the price of land. 
Each agent has a residential and an occupational choice. $r(e) \in J$ is the residential choice of agent $e$, where $r(e)=j$ indicates that agent $e$ decides to reside in neighborhood $j . o(e) \in\left\{w, b_{1}, \ldots, b_{J}\right\}$ is the occupational choice of agent $e$, where $o(e)=w$ indicates that $e$ is a worker and $o(e)=b_{j}$ that $e$ is a criminal who operates in neighborhood $j$. Let $N(o, j)=\left\{e \in\left[e^{L}, e^{H}\right]: o(e)=o\right.$ and $\left.r(e)=j\right\}$ be the set of agents who choose occupation $o$ and reside in $j$ and $N(o)=\left\{e \in\left[e^{L}, e^{H}\right]: o(e)=o\right\}$ be the set of agents who choose occupation $o^{9}$ Criminals extract income from workers. Let $\gamma_{j}:\left[e^{L}, e^{H}\right] \rightarrow[0,1]$ indicate for each agent $e \in\left[e^{L}, e^{H}\right]$ the fraction of $e$ 's income that criminals will extract if $e$ resides in neighborhood $j$ and chooses to be a worker. $\gamma_{j}$ is partially given by technology and partially determined by the government. In particular, assume that $\gamma_{j}(e)=\left(1-p_{j}\right) \gamma(e)$, where $\gamma(e)$ is the fraction of $e$ 's income extracted by criminals when the government does not intervene, and $p_{j}$ represents the protection strategy chosen by the government. The government selects one of the following protection strategies: under concentrated protection $p_{j}=1$ for $j \in J_{p}$ and $p_{j}=0$ for $j \in J_{u n}=J-J_{p}$, under dispersed protection $p_{j}=p<1$ for all $j \in J$.

Let $y^{e}(o(e), r(e))$ indicate the income of agent $e$ when he or she selects $(o(e), r(e))$. If $e$ becomes a worker and resides in $j$, then

$$
y^{e}(w, j)=\left(1-\gamma_{j}(e)\right)\left[\bar{p}_{C} e+s_{L}(e) \sum_{j \in J} p_{L, j} \bar{L}_{j}\right]
$$

If $e$ becomes a criminal who operates in neighborhood $j$, then

$$
\begin{aligned}
y^{e}\left(b_{j}\right) & =\epsilon(e)\left\{\delta \int_{N(w, j)} \gamma_{j}(e)\left[\bar{p}_{C} e+s_{L}(e) \sum_{j \in J} p_{L, j} \bar{L}_{j}\right] f(e) d e\right\}, \text { and } \\
\epsilon(e) & =\epsilon\left[\int_{N\left(b_{j}\right)} f(e) d e\right]^{-1}+(1-\epsilon) e\left[\int_{N\left(b_{j}\right)} e f(e) d e\right]^{-1} .
\end{aligned}
$$

The term in brackets is total plunder in neighborhood $j$ (note that $(1-\delta) \in(0,1)$ of it is lost), while $\epsilon(e)$ captures how criminals operating in neighborhood $j$ distribute the plunder. A fraction $\epsilon \in[0,1]$ is distributed evenly among criminals. The rest depends on the labor endowment of the criminal relative to the average labor endowment of all criminals. Thus, better endowed criminals get a higher share of the total plunder.

Regardless of how they obtain their income, agents demand housing services and consumption goods. Assume that all have the same utility function $v^{e}\left(o(e), r(e), h_{r(e)}^{e}, c_{r(e)}^{e}\right)=\left(\frac{h_{r(e)}^{e}}{\alpha}\right)^{\alpha}\left(\frac{c_{r(e)}^{e}}{1-\alpha}\right)^{1-\alpha}$, where

\footnotetext{
${ }^{9}$ Note that $N(o)=\cup_{j \in J} N(o, j)$.
} 
$h_{r(e)}^{e}$ is housing services and $c_{r(e)}^{e}$ is consumption. The budget constraint of agent $e$ is $p_{H, r(e)} h_{r(e)}^{e}+$ $\bar{p}_{C} c_{r(e)}^{e}=y^{e}(o(e), r(e))$. Finally, we introduce the following assumption:

Assumption 1.C. $\gamma(e)$ and $\theta(e)=e\left[1-\left(1-p_{j}\right) \gamma(e)\right]$ are strictly increasing functions of $e$. That is, the better endowed an agent is, the more vulnerable he or she is to criminal activities. However, this is not enough to affect the income ranking of agents.

Given the protection regime and the prices of tradable goods, an equilibrium is land prices $p_{L, j}$, residential and occupational decisions $N(o, r)$, and income levels $y^{e}(o(e), r(e))$ such that: (1) Income levels are given by equations (19)-(21). (2) Given $p_{L, j}$, residential and occupational decisions are given by:

$$
N(o, r)=\left\{e \in\left[e^{L}, e^{H}\right]: \frac{y^{e}(o, r)}{\left(p_{L, r}\right)^{\alpha\left(1-\beta_{K, H}\right)}} \geq \frac{y^{e}\left(o^{\prime}, r^{\prime}\right)}{\left(p_{L, r^{\prime}}\right)^{\alpha\left(1-\beta_{K, H}\right)}} \text { for all }\left(o^{\prime}, r^{\prime}\right)\right\} .
$$

(3) Housing markets clear, that is,

$$
\frac{p_{L, j} \bar{L}_{j}}{1-\beta_{K, H}}=\alpha\left[\int_{N(w, j)} y^{e}(w, j) f(e) d e+\sum_{h \in J} \int_{N\left(b_{h}, j\right)} y^{e}\left(b_{h}\right) f(e) d e\right] .
$$

\subsection{Concentrated Protection}

Suppose that police protection is concentrated. Let $\bar{L}_{u n}=\sum_{j \in J_{u n}} \bar{L}$ and $\bar{L}_{p}=\sum_{j \in J_{p}} \bar{L}_{j}$ be the land in unprotected and protected neighborhoods, respectively. $N\left(J_{u n}, J_{u n}\right)$ denotes the set of agents who become criminals operating and residing in unprotected neighborhoods $\left(J_{u n}\right) ; N\left(w, J_{u n}\right)$ denotes the set of agents who choose to work and reside in $J_{u n}$; and $N\left(w, J_{p}\right)$ denotes the set of agents who choose to work and reside in protected neighborhood $\left(J_{p}\right)$. We search for an equilibrium in which $N\left(J_{u n}, J_{u n}\right)=\left[e_{L}, e_{C}^{w}\right)$, $N\left(w, J_{u n}\right)=\left[e_{C}^{w}, e_{C}^{u n}\right)$, and $N\left(w, J_{p}\right)=\left[e_{C}^{u n}, e_{H}\right]$, where $\bar{e}>e_{C}^{u n}>e_{C}^{w}>e_{L}$. Lemma 4 charactresizes this equilibrium.

Lemma 4 Suppose that protection is concentrated, Assumption 1.C holds, $\frac{\bar{L}_{p}}{\bar{L}_{u n}}>\frac{\alpha\left(1-\beta_{K, H}\right)}{1-\alpha\left(1-\beta_{K, H}\right)}$ and $\frac{e(1-\gamma(e))}{\epsilon(e)}$ is increasing in $e$. Then, there is an equilibrium in which $N\left(J_{u n}, J_{u n}\right)=\left[e_{L}, e_{C}^{w}\right), N\left(w, J_{u n}\right)=$ $\left[e_{C}^{w}, e_{C}^{u n}\right)$, and $N\left(w, J_{p}\right)=\left[e_{C}^{u n}, e_{H}\right]$, where $e_{L}<e_{C}^{w}<e_{C}^{u n}<\bar{e}<e_{H}$ and $e_{C}^{w}$ and $e_{C}^{u n}$ are uniquely 
determined by:

$$
\begin{aligned}
& \frac{\left[1-\gamma\left(e_{C}^{u n}\right)\right]^{\frac{1}{\alpha\left(1-\beta_{K, H}\right)}}}{\left[1-\alpha\left(1-\beta_{K, H}\right)\right] \int_{e_{C}^{u n}}^{e_{C}^{u n}} \eta_{C}(e) e f(e) d e}+\frac{\alpha\left(1-\beta_{K, H}\right)}{1-\alpha\left(1-\beta_{K, H}\right)}-\frac{\bar{L}_{p}}{\bar{L}_{u n}}=0 \\
& \frac{e_{C}^{w}\left(1-\gamma\left(e_{C}^{w}\right)\right)}{\epsilon\left(e_{C}^{w}\right)}-\delta \int_{e_{C}^{w}}^{e_{u}^{u n}} \gamma(e) e f(e) d e=0
\end{aligned}
$$

Moreover, in this equilibrium, land prices are given by:

$$
\begin{aligned}
p_{L_{u n}} & =\alpha\left(1-\beta_{K, H}\right)\left(\bar{L}_{u n}\right)^{-1} \bar{p}_{C} \int_{e_{C}^{w}}^{e_{u}^{u n}} \eta_{C}(e) e f(e) d e \\
p_{L_{p}} & =\frac{\alpha\left(1-\beta_{K, H}\right)\left(\bar{L}_{p}\right)^{-1} \bar{p}_{C}}{1-\alpha\left(1-\beta_{K, H}\right)}\left[\int_{e_{C}^{u n}}^{e^{H}} \operatorname{ef}(e) d e+\alpha\left(1-\beta_{K, H}\right) \int_{e_{C}^{w}}^{e_{C}^{u n}} \eta_{C}(e) e f(e) d e\right]
\end{aligned}
$$

Proof: See Online Appendix.

Lemma 4 simply states that when protection is concentrated, the city is segregated. Only very poor agents become criminals. Poor agents (criminals and workers) locate in high-crime neighborhoods with low housing prices $\left(J_{u n}\right)$, while rich agents locate in safe neighborhoods with high housing prices $\left(J_{p}\right)$. There are two marginal agents. The agent with labor endowment $e_{C}^{w}$ is indifferent between becoming a criminal who operates in the unprotected area or being a worker. The agent with labor endowment $e_{C}^{u n}$ is indifferent between residing in the protected and the unprotected area.

\subsection{Dispersed Protection}

Suppose that protection is evenly dispersed in both neighborhoods. We search for an equilibrium in which $p_{L, j}=p_{L}$ for all $j \in J$, agents with $e \in N(b)=\left[e_{L}, e_{D}^{w}\right)$ are criminals, agents with $e \in N(w)=\left[e_{D}^{w}, e_{H}\right]$ are workers, $\bar{e}>e_{D}^{w}>e_{L}$, and the proportion of agents of type $e$ in neighborhood $j$ is $\bar{L}_{j} / \bar{L}$. Lemma 5 characterizes this equilibrium.

Lemma 5 Suppose that protection is evenly distributed in both neighborhoods and Assumption 1.C holds. Then, there is an equilibrium in which $N(b)=\left[e_{L}, e_{D}^{w}\right)$ and $N(w)=\left[e_{D}^{w}, e_{H}\right]$, with $\bar{e}>e_{D}^{w}>e_{L}$ and $e_{D}^{w}$ determined by:

$$
\frac{e_{D}^{w}\left[1-(1-p) \gamma\left(e_{D}^{w}\right)\right]}{\epsilon\left(e_{D}^{w}\right)}-\delta(1-p)\left[\begin{array}{c}
\int_{e_{D}^{w}}^{e^{H}} \gamma(e) e f(e) d e+ \\
\alpha\left(1-\beta_{K, H}\right)\left(\int_{e^{L}}^{e^{H}} \eta_{D}(e) e f(e) d e\right)\left(\int_{e_{D}^{w}}^{e^{H}} \gamma(e) s_{L}(e) f(e) d e\right) \\
\left.+1-\alpha\left(1-\beta_{K, H}\right) \int_{e^{L}}^{e^{H}} \eta_{D}(e) s_{L}(e) f(e) d e\right]
\end{array}\right]=0
$$


Moreover, if $\frac{e[1-(1-p) \gamma(e)]}{\epsilon(e)}$ is increasing in e, there is a unique $e_{D}^{w}$ that solves equation (28). In equilibrium, the price of land is given by

$$
p_{L}=\frac{\alpha\left(1-\beta_{K, H}\right) \bar{p}_{C}(\bar{L})^{-1} \int_{e^{L}}^{e^{H}} \eta_{D}(e) e f(e) d e}{1-\alpha\left(1-\beta_{K, H}\right) \int_{e^{L}}^{e^{H}} \eta_{D}(e) s_{L}(e) f(e) d e}
$$

Proof: See Online Appendix.

Lemma 5 shows that when protection is evenly distributed across all neighborhoods, the city becomes integrated. In equilibrium, income per capita and crime levels are equalized in all neighborhoods and agents are indifferent with respect to their residential decision. There is a marginal agent with labor endowment $e_{D}^{w}$ who is indifferent between being a criminal or a worker. Only agents poorer with $e<e_{D}^{w}$ become criminals who operate in the entire city, while the rest choose to work.

\subsection{Regime Comparisons}

Next, we compare several key variables under concentrated and dispersed protection. We focus on crime, income, housing prices, and welfare. For aggregate welfare comparisons we assume a utilitarian welfare function, that is, $W=\int_{e_{L}}^{e_{H}} v^{e} f(e) d e$, where $v^{e}$ is the utility obtained by an agent with labor endowment $e$. Define $\Gamma_{C}^{u n}=$ $\left[\int_{e_{C}^{w}}^{e_{C}^{u n}} \eta_{C}(e) e f(e) d e\right]^{1-\alpha\left(1-\beta_{K, H}\right)}, \Gamma_{C}^{p}=\left[\frac{\alpha\left(1-\beta_{K, H}\right) \int_{e_{C}^{w}}^{e_{C}^{u n}} \eta_{C}(e) e f(e) d e+\int_{e_{C}^{u n}}^{e^{H}} e f(e) d e}{1-\alpha\left(1-\beta_{K, H}\right)}\right]^{1-\alpha\left(1-\beta_{K, H}\right)}$, , and $\Gamma_{D}=$ $\left[\frac{\int_{e^{L}}^{e^{H}} \eta_{D}(e) e f(e) d e}{1-\alpha\left(1-\beta_{K, H}\right) \int_{e^{L}}^{e^{H}} \eta_{D}(e) s_{L}(e) f(e) d e}\right]^{1-\alpha\left(1-\beta_{K, H}\right)}$. The following proposition summarizes the results:

Proposition 3 Under the assumption and conditions in Lemmas 3 and 4.

1. Crime, measured as the value of the goods stolen, is lower under concentrated than under dispersed protection if and only if $\frac{e_{C}^{w}\left(1-\gamma\left(e_{C}^{w}\right)\right)}{\epsilon\left(e_{C}^{w}\right)}<\frac{e_{D}^{w}\left[1-(1-p) \gamma\left(e_{D}^{w}\right)\right]}{\epsilon\left(e_{D}^{w}\right)}$.

2. Aggregate income under concentrated protection is higher than under dispersed protection if and only if $\left(\Gamma_{C}^{u n}\right)^{\frac{1}{1-\alpha\left(1-\beta_{K, H}\right)}}+\left(\Gamma_{C}^{p}\right)^{\frac{1}{1-\alpha\left(1-\beta_{K, H}\right)}}>\left(\Gamma_{D}\right)^{\frac{1}{1-\alpha\left(1-\beta_{K, H}\right)}}$.

3. Housing prices under dispersed protection are between housing prices in unprotected and protected neighborhoods under concentrated protection $\left(p_{L_{u n}}<p_{L}<p_{L_{p}}\right)$ if and only if $\left(\bar{L}_{u n}\right)^{-1}\left(\Gamma_{C}^{u n}\right)^{\frac{1}{1-\alpha\left(1-\beta_{K, H}\right)}}<(\bar{L})^{-1}\left(\Gamma_{D}\right)^{\frac{1}{1-\alpha\left(1-\beta_{K, H}\right)}}<\left(\bar{L}_{p}\right)^{-1}\left(\Gamma_{C}^{p}\right)^{\frac{1}{1-\alpha\left(1-\beta_{K, H}\right)}}$. 
4. Aggregate welfare under concentrated protection is higher than under dispersed protection, i.e., $W_{C}>W_{D}$, if and only if $\Gamma_{C}^{u n}\left(\frac{\bar{L}_{u n}}{\bar{L}}\right)^{\alpha\left(1-\beta_{K, H}\right)}+\Gamma_{C}^{p}\left(\frac{\bar{L}_{p}}{\bar{L}}\right)^{\alpha\left(1-\beta_{K, H}\right)}<\Gamma_{D}$. Proof: See Online Appendix.

Proposition 3 is much less informative than Propositions 1 and 2. The reason is that the conditions in Proposition 3 depend on endogenous variables, that is, $e_{C}^{w}$, $e_{C}^{u n}$, and $e_{D}^{w}$. Note, however, that in order to compare crime statistics between the two protection regimes we only need to know $e_{C}^{w}$ and $e_{D}^{w}$. Moreover, if $e\left(1-\gamma_{j}(e)\right) / \epsilon(e)$ is increasing in $e$, then the two ways of measuring crime will induce the same comparison. If crime is higher under concentrated than dispersed protection according to one crime statistic, the same will be true for the other. Note also that, in order to compare aggregate income, housing prices and aggregate welfare under concentrated and dispersed protection we only need $e_{C}^{w}$ and $e_{C}^{u n}$, while $e_{D}^{w}$ does not play any role. This suggests that crime statistics could be a misleading way to infer welfare changes.

\section{Conclusions}

This paper has developed a general equilibrium model of the geographic distribution of crime in an urban area. In order to characterize the equilibrium we have repeatedly applied the notion of spatial equilibrium. This implies that the equilibrium geographic distribution of legal and illegal activities must be such that there are no gains from changing their location. We have employed the model to study police deployment strategies. To the best of our knowledge, this paper is the first to analytically explore the consequences of police deployment strategies on the endogenous formation of urban spatial equilibrium and crime. Our formal general equilibrium approach model is pertinent because a change in the geographic distribution of police protection induces long-run general equilibrium effects on occupational and residential choices, the location of criminal activities, and housing prices. Indeed, our model revealed two channels through which the spatial allocation of police protection affects individuals. First, it changes the intensity and location of crime, thus affecting the income of each agent. Second, it changes housing prices.

In particular, this paper has examined two opposite police deployment strategies: concentrated public protection (the police only protect a certain area of the city), and dispersed public protection (the police are evenly deployed across the entire the city). We have shown that, in equilibrium, concentrated public 
protection leads to a segregated city. The rich, who are more willing to pay for protection, reside in protected areas with high housing prices. The poor reside in unprotected high-crime areas with low housing prices. Conversely, dispersed public protection leads to an integrated city, with all areas of the city having the same income per capita and crime levels. We have also compared these two equilibria and shown that crime tends to be higher under dispersed than concentrated public protection when inequality is high, dispersing the police force significantly reduces its effectiveness, and the proportion of income that criminals extract from the rich (poor) is high (low). We have also shown that in very unequal societies concentrated public protection is likely to produce higher aggregate welfare but exacerbate social disparities. Fortunately, there is a set of taxes and subsidies that the policymaker can use to avoid the unfair distributive effects associated with concentrated public protection. Thus, instead of dispersing the police, taxing the rich and properly redistributing the proceeds to the group made worse off by police concentration has the potential to efficiently equalize the cost of crime in society. Moreover, we have shown that dispersing the police might not necessarily produce an integrated city when private security is available. If private security is a club good, rich agents have incentives to isolate themselves in closed residential areas.

Besides private security, there are several other directions in which our model can be extended. For example, it is reasonable to assume that the geographic distribution of protection could affect schooling decisions, particularly for poor agents. One way to capture this interaction is to imbed our model in a dynamic human capital formation model. We conjecture that in this setting dispersed protection is more likely to produce higher aggregate welfare than in our model. The reason is that dispersed protection would promote schooling among poor agents without significantly affecting schooling among rich agents.

Our model also suggests new paths for empirical work on the economics of crime and law enforcement. For example, we have a very limited understanding of the effectiveness of more concentrated versus more dispersed allocations of police forces. Some work on this issue has been done related to the literature on hot spots (Weisburd, Groff, and Yang, 2012). The next empirical step would be a consistent estimation of the parameters of the production function of security across locations. Our model provides a theoretical framework for such an estimation.

Finally, our model points to some interesting policy recommendations. The literature on crime hot spots has found evidence that they tend to be quite stable over time, which suggests concentrating 
police efforts on those hot areas. Our results indicate that one needs to be cautious about such law enforcement strategies. For example, in our model, ceteris paribus the total area protected, changing the neighborhoods in which the police are concentrated only switches the entire configuration of the spatial equilibrium, with no long-run effect on crime and welfare. The new protected neighborhoods become rich safe heavens, while the previously prosperous neighborhoods now left unprotected become the new criminal hot spots. Moreover, our model does not take into account any transition costs, which could easily make this policy a welfare-reducing one. At a minimum, our model suggests that when we consider changes in the geographic distribution of police forces, we need to take into account the effects on housing prices and on reallocation of the population, as well as the overall effect on crime in the entire city (see Jaitman and Ajzenman, 2016, for evidence of these effects in Montevideo, Uruguay).

\section{References}

Ajzenman, N., S. Galiani, and E. Seira. 2015. On the Distributive Costs of Drug-Related Homicides. Journal of Law and Economics.

Banerjee, A., E. Duflo, D. Keniston, and N. Singh. 2012. Crime, Punishment, and Monitoring: Deterring Drunken Driving in India. Manuscript.

Becker, G.S. 1968. Crime and Punishment: An Economic Approach. Journal of Political Economy 76(2): 169-217.

Benabou, R. 1993. Workings of a City: Location, Education, and Production. Quarterly Journal of Economics, 108: 619-652.

Benabou, R. 1996. Equity and Efficiency in Human Capital Investment: The Local Connection. Review of Economic Studies 62: 237-264.

Benson, B.L., and P.R. Zimmerman. 2010. Handbook on the Economics of Crime. Edward Elgar Publishing.

Benson, B.L. 2010. The allocation of police. Benson, B.L., and P.R. Zimmerman (eds.), Handbook on the Economics of Crime. Edward Elgar Publishing.

Calabrese, S., D. Epple., H. Sieg, and T. Romer. 2006. Local Public Good Provision: Voting, Peer Effects, and Mobility. Journal of Public Economics 90(6-7): 959-81. 
Cook, P.J. 2009. The Economics of Crime: An Introduction to Rational Crime Analysis. Journal of Economic Literature 47(3): 804-806.

Cook, P. J., S. Machin, O. Marie, and G. Mastrobuomi. 2013. Crime Economics in its Fifth Decade, in Cook, Machin, Marie and Mastrobuomi (eds.), Lessons from the Economics of Crime, MIT Press.

Demombynesa, G., and B. Ozler. 2005. Crime and Local Inequality in South Africa. Journal of Development Economics 76: 265-292.

Di Tella, R., S. Galiani, and E. Schargrodsky. 2010. Crime Distribution and Victim Behavior during a Crime Wave. In The Economics of Crime: Lessons from Latin America, edited by R. Di Tella, S. Edwards, and E. Schargrodsky. Cambridge, MA: NBER.

Di Tella, R., and E. Schargrodsky. 2004. Do Police Reduce Crime? Estimates Using the Allocation of Police Forces after a Terrorist Attack. American Economic Review 94(1): 115-33.

Draca, M., and S. Machin. 2015. Crime and Economics Incentives. Annual Review of Economics 389-408.

Draca, M., S. Machin, and R. Witt. 2011. Panic on the Streets of London: Police, Crime, and the July 2005 Terror Attacks. American Economic Review 101(5): 2157-181.

Eeckhout, J., N. Persico, and P. E. Todd. 2010. A Theory of Optimal Random Crackdowns. American Economic Review, 100 (3):1104-35.

Evans, W.N., and E.G. Owens. 2007. COPS and Crime. Journal of Public Economics 91(1): $181-01$.

Fu, C. and K. Wolpin. 2015. Structural Estimation of a Becker-Ehrlich Equilibrium Model of Crime: Allocating Police Across Cities to Reduce Crime. RISE Working Paper 14-020.

Glaeser, E. 1999. An Overview of Crime and Punishment. Harvard University. Unpublished.

Glaeser, E. 2008. Cities, Agglomeration and Spatial Equilibrium. Oxford: Oxford University Press.

Hirshleifer, J. 1991. The Paradox of Power. Economics and Politics 3: 177-200.

Jaitman, L., and N. Ajzenman. 2016. Crime Concentration and Hotspot Dynamics in Latin America. Inter-American Development Bank. Unpublished.

Kang, S. 2016. Inequality and Crime Revisited: Effects of Local Inequality and Economic Segregation on Crime. Journal of Population Economics, forthcoming. 
Klick, J., and A. Tabarrok. 2005. Using Terror Alert Levels to Estimate the Effect of Police on Crime. Journal of Law and Economics 48(1): 267-79.

Levitt, S. 1997. Using Electoral Cycles in Police Hiring to Estimate the Effect of Police on Crime. American Economic Review 87(3): 270-90.

Levitt, S. 1999. The Changing Relationship between Income and Crime Victimization. FRBNY Economic Policy Review (September): 87-99.

Levitt, S. 2004. Understanding Why Crime Fell in the 1990s: Four Factors that Explain the Decline and Six that Do Not. Journal of Economic Perspectives 18(1): 163-90.

Linden, L., and J. Rockoff. 2008. Estimates of the Impact of Crime Risk on Property Values from Megan's Laws. American Economic Review 98(3): 1103-127.

Lum, C., and D.S. Nagin. 2015. Reinventing American Policing: A Six-Point Blueprint for the 21st Century. Transnational Criminology (Fall): 2-5.

Quy-Toan D., L. Ma, and C. Ruiz. 2016. Pirates of Somalia: Crime and Deterrence on the High Seas. Unpublished.

Verdier, T., and Y. Zenou. 2004. Crime, Location and Beliefs. International Economic Review 45(3): 731-760.

Weisburd, D., E. Groff, and S. Yang. 2012. The Criminology of Place: Street Segments and Our Understanding of the Crime Problem. Oxford: Oxford University Press.

Yezer, A. 2014. Economics of Crime and Enforcement. London and New York: Routledge. 


\section{Online Appendix to "Stirring Up a Hornets' Nest: Geographic Distri- bution of Crime"}

This appendix proves all the lemmas and propositions outlined in the main text.

Proof of Lemma 1. Suppose that protection is concentrated. Assume that $o^{s}=w$ and $r^{s} \in J_{p}$. Then, in equilibrium, $N^{s}(w, j)=\left(\bar{L}_{j} / \bar{L}_{p}\right) N^{s}$ and $p_{H, j}=p_{H_{p}}$ for $j \in J_{p}$. Otherwise, some agents in $s$ prefer to change their residence to a neighborhood in $J_{p}$ with lower $p_{H, j}$. Let $B_{u n}=\left\{b_{j} \in O: j \in J_{u n}\right\}$ and assume that $o^{l} \in B_{u n}, o^{i}=w$ for $i \in\{m, h\}$, and $r^{i} \in J_{u n}$ for $i \in\{l, m, h\}$. Then, in equilibrium, $N^{h}(w, j)=\left(\bar{L}_{j} / \bar{L}_{u n}\right) N^{h}, N^{m}(w, j)=\left(\bar{L}_{j} / \bar{L}_{u n}\right) N^{m}, N^{l}\left(b_{j}, J_{u n}\right)=\sum_{h \in J_{u n}} N^{l}\left(b_{j}, h\right)=N^{l}\left(B_{u n}, j\right)=$ $\sum_{h \in J_{u n}} N^{l}\left(b_{h}, j\right)=\left(\bar{L}_{j} / \bar{L}_{u n}\right) N^{l}$, and $p_{H, j}=p_{H_{u n}}$ for $j \in J_{u n}$. Otherwise, some agents in $i \in\{l, m, h\}$ prefer to change their residence to a neighborhood in $J_{u n}$ with lower $p_{H, j}$, some agents in group $i \in\{m, h\}$ prefer to move to a neighborhood in $J_{u n}$ with lower crime, or some agents in group $l$ prefer to change their criminal activities to a neighborhood in $J_{u n}$ with higher income per criminal.

Given these occupational choices we have $U_{C}=\sum_{i=m, h} N^{i} \bar{e}^{i}, S_{C}=N^{s} \bar{e}^{s}$, and $Q_{C}=$ $\left(U_{C}\right)^{\beta_{U, C}}\left(S_{C}\right)^{1-\beta_{U, C}}$. Then, from profit maximization in industry $C, w^{u}=\bar{p}_{C} \beta_{U, C}\left(S_{C} / U_{C}\right)^{1-\beta_{U, C}}$, $w^{s}=\bar{p}_{C}\left(1-\beta_{U, C}\right)\left(U_{C} / S_{C}\right)^{\beta_{U, C}}$, where $\bar{p}_{C}>0$. Moreover, incomes are given by: $y^{l}\left(b_{h}, j\right)=y^{l}=$ $\delta w^{u}\left(N^{l}\right)^{-1} \sum_{i=m, h} \gamma^{i} N^{i} \bar{e}^{i}$ for $h, j \in J_{u n}, y^{m}(w, j)=y^{m}=\left(1-\gamma^{m}\right) w^{u} \bar{e}^{m}$ and $y^{h}(w, j)=y^{h}=$ $\left(1-\gamma^{h}\right) w^{u} \bar{e}^{h}$ for $j \in J_{u n}$, and $y^{s}(w, j)=y^{s}=w^{s} \bar{e}^{s}+\left(N^{s}\right)^{-1} \sum_{j \in J} p_{L, j} \bar{L}_{j}$ for $j \in J_{p}$.

From profit maximization in industry $H, \quad K_{H, j}=\beta_{K, H} p_{L, j} \bar{L}_{j} /\left(1-\beta_{K, H}\right) \bar{p}_{K}, \quad p_{H, j}=$ $\left(\bar{p}_{K} / \beta_{K, H}\right)^{\beta_{K, H}}\left[p_{L, j} /\left(1-\beta_{K, H}\right)\right]^{1-\beta_{K, H}}$, and $p_{H, j} Q_{H, j}=p_{L, j} \bar{L}_{j} /\left(1-\beta_{K, H}\right)$, where $\bar{p}_{K}>0$ is the price of capital. Since $p_{H, j}=p_{H_{p}}$ for $j \in J_{p}$ and $p_{H, j}=p_{H_{u n}}$ for $j \in J_{u n}$, it must be the case that $p_{L, j}=p_{L_{p}}$ for $j \in J_{p}$ and $p_{L, j}=p_{L_{u n}}$ for $j \in J_{u n}$. Let $Q_{H_{p}}=\sum_{j \in J_{p}} Q_{H, j}$ and $Q_{H_{u n}}=\sum_{j \in J_{u n}} Q_{H, j}$. Then, the market clearing conditions in the housing markets are $p_{H_{u n}} Q_{H_{u n}}=\alpha \sum_{i=l . m . h} y^{i} N^{i}$ and $p_{H_{p}} Q_{H_{p}}=\alpha y^{s} N^{s}$. Introducing housing supplies and the income of each group into these market clearing conditions, equilibrium land prices are:

$$
\begin{aligned}
p_{L_{u n}} & =\alpha\left(1-\beta_{K, H}\right) \beta_{U, C} \bar{p}_{C} Q_{C}\left(\bar{L}_{u n}\right)^{-1} \sum_{i=m, h} \eta_{C}^{i} n^{i} \\
p_{L_{p}} & =\alpha\left(1-\beta_{K, H}\right) \beta_{U, C} \bar{p}_{C} Q_{C}\left(\bar{L}_{p}\right)^{-1}\left[\frac{\left(1-\beta_{U, C}\right)+\alpha\left(1-\beta_{K, H}\right) \beta_{U, C} \sum_{i=m, h} \eta_{C}^{i} n^{i}}{\beta_{U, C}\left[1-\alpha\left(1-\beta_{K, H}\right)\right]}\right]
\end{aligned}
$$

where $n^{i}=\bar{e}^{i} N^{i}\left(\sum_{i=m, h} \bar{e}^{i} N^{i}\right)^{-1}$ for $i=m, h$ and $\eta_{C}^{i}=1-\gamma^{i}+\delta \gamma^{i}$. Equilibrium housing prices 
are $p_{H_{u n}}=\left(\bar{p}_{K} / \beta_{K, H}\right)^{\beta_{K, H}}\left[p_{L_{u n}} / 1-\beta_{K, H}\right]^{1-\beta_{K, H}}$ and $p_{H_{p}}=\left(\bar{p}_{K} / \beta_{K, H}\right)^{\beta_{K, H}}\left[p_{L_{p}} /\left(1-\beta_{K, H}\right)\right]^{1-\beta_{K, H}}$. Thus, $p_{H_{p}}>p_{H_{u n}}$ if and only if $p_{L_{p}}>p_{L_{u n}}$.

Finally, it still has to be checked that no agent has an incentive to select the occupation and/or residence selected by an agent in a different group. The indirect utility of an agent in group $i$ who selects $\left(o^{i}, r^{i}\right)$ is $v^{i}\left(o^{i}, r^{i}\right)=y^{i}\left(o^{i}, r^{i}\right)\left[\left(p_{H, r^{i}}\right)^{\alpha}\left(\bar{p}_{C}\right)^{1-\alpha}\right]^{-1}$. Therefore, we have:

Group $l$. Agents in group $l$ do not want to change their criminal operations to $j \in J_{p}$ because they would get no income. They prefer to reside in $J_{u n}$ rather than in $J_{p}$ provided that $p_{L_{p}}>p_{L_{u n}}$. They prefer to be criminals rather than workers if $\delta\left(N^{l}\right)^{-1} \sum_{i=m, h} \gamma^{i} N^{i} \bar{e}^{i}>\bar{e}^{l} \max \left\{\left(1-\gamma^{l}\right),\left(p_{L_{u n}} / p_{L_{p}}\right)^{\alpha\left(1-\beta_{K, H}\right)}\right\}$.

Group $i \in\{m, h\}$. Agents in group $i$ prefer to be workers rather than criminals if $\left(1-\gamma^{i}\right) \bar{e}^{i}>$ $\delta\left(N^{l}\right)^{-1} \sum_{i=m, h} \gamma^{i} N^{i} \bar{e}^{i} \max \left\{1,\left(p_{L_{u n}} / p_{L_{p}}\right)^{\alpha\left(1-\beta_{K, H}\right)}\right\}$. They prefer to reside in $J_{u n}$ rather than in $J_{p}$ if $1-\gamma^{i}>\left(p_{L_{u n}} / p_{L_{p}}\right)^{\alpha\left(1-\beta_{K, H}\right)}$.

Group s. Agents in group $s$ prefer to be workers rather than criminals if $w^{s} \bar{e}^{s}>w^{u} \delta\left(N^{l}\right)^{-1} \sum_{i=m, h} \gamma^{i} N^{i} \bar{e}^{i} \quad$ and $w^{s} \bar{e}^{s}+\left(N^{s}\right)^{-1}\left(p_{L_{u n}} \bar{L}_{u n}+p_{L_{p}} \bar{L}_{p}\right)>$ $\left(p_{L_{p}} / p_{L_{u n}}\right)^{\alpha\left(1-\beta_{K, H}\right)}\left[w^{u} \delta\left(N^{l}\right)^{-1} \sum_{i=m, h} \gamma^{i} N^{i} \bar{e}^{i}+\left(1-\gamma^{s}\right)\left(N^{s}\right)^{-1}\left(p_{L_{u n}} \bar{L}_{u n}+p_{L_{p}} \bar{L}_{p}\right)\right] . \quad$ They prefer to reside in $J_{p}$ rather than in $J_{u n}$ if $1-\gamma^{s}<\left(p_{L_{u n}} / p_{L_{p}}\right)^{\alpha\left(1-\beta_{K, H}\right)} \cdot{ }^{10}$

Employing Assumption 1, these conditions simplify to $\left(1-\gamma^{l}\right) \bar{e}^{l}<\delta\left(N^{l}\right)^{-1} \sum_{i=m, h} \gamma^{i} N^{i} \bar{e}^{i}<$ $\left(1-\gamma^{m}\right) \bar{e}^{m}$ and $1-\gamma^{s}<\left(p_{L_{u n}} / p_{L_{p}}\right)^{\alpha\left(1-\beta_{K, H}\right)}<1-\gamma^{h}$. This completes the proof of Lemma 1.

Proof of Lemma 2. Suppose that protection is dispersed. Assume that $o^{l} \in B=O-\{w\}$, $o^{i}=w$ for $i \in\{m, h, s\}$ and $r^{i} \in J$ for $i \in I$. Then, in equilibrium, $N^{l}\left(b_{j}, J\right)=\sum_{h \in J} N^{l}\left(b_{j}, h\right)=$ $N^{l}(B, j)=\sum_{h \in J} N^{l}\left(b_{h}, j\right)=\left(\bar{L}_{j} / \bar{L}\right) N^{l}, N^{i}(w, j)=\left(\bar{L}_{j} / \bar{L}\right) N^{i}$ for $i \in\{m, h, s\}$, and $p_{H, j}=p_{H}$ for $j \in J$. Otherwise, some agents in $i \in I$ prefer to change their residence to a neighborhood with lower $p_{H, j}$, some agents in group $i \in\{m, h, s\}$ prefer to move to a neighborhood with lower crime, or some agents in group $l$ prefer to change their criminal activities to a neighborhood with higher income per criminal.

\footnotetext{
${ }^{10}$ We are implicitly assuming that if a skilled worker decides to become a criminal, he or she keeps his/her land holdings. Alternatively, if he or she also loses his/her land holdings, conditions become: $w^{s} \bar{e}^{s}++\left(N^{s}\right)^{-1}\left(p_{L_{u n}} \bar{L}_{u n}+p_{L_{p}} \bar{L}_{p}\right)>$ $w^{u} \delta\left(N^{l}\right)^{-1} \sum_{i=m, h} \gamma^{i} N^{i} \bar{e}^{i}$ and $w^{s} \bar{e}^{s}+\left(N^{s}\right)^{-1}\left(p_{L_{u n}} \bar{L}_{u n}+p_{L_{p}} \bar{L}_{p}\right)>\left(\frac{p_{L_{p}}}{p_{L_{u n}}}\right)^{\alpha\left(1-\beta_{K, H}\right)} w^{u} \delta\left(N^{l}\right)^{-1} \sum_{i=m, h} \gamma^{i} N^{i} \bar{e}^{i}$. They prefer to reside in $J_{p}$ rather than in $J_{u n}$ if $1-\gamma^{s}<\left(\frac{p_{L_{u n}}}{p_{L_{p}}}\right)^{\alpha\left(1-\beta_{K, H}\right)}$. In any case, under Assumption 1 , only the last condition may be binding.
} 
Given these occupational choices we have $U_{C}=\sum_{i=m, h} N^{i} \bar{e}^{i}, S_{C}=N^{s} \bar{e}^{s}$ and $Q_{C}=$ $\left(U_{C}\right)^{\beta_{U, C}}\left(S_{C}\right)^{1-\beta_{U, C}}$. Then, from profit maximization in industry $C, w^{u}=\bar{p}_{C} \beta_{U, C}\left(S_{C} / U_{C}\right)^{1-\beta_{U, C}}$, $w^{s}=\bar{p}_{C}\left(1-\beta_{U, C}\right)\left(U_{C} / S_{C}\right)^{\beta_{U, C}}$, where $\bar{p}_{C}>0$. Moreover, incomes are given by $y^{l}\left(b_{h}, j\right)=y^{l}=$ $\left(N^{l}\right)^{-1} \delta \sum_{i=m, h, s} \gamma_{D}^{i} N^{i}\left[w^{i} \bar{e}^{i}+s_{L}^{i} \sum_{j \in J} p_{L, j} \bar{L}_{j}\right]$ and $y^{i}(w, j)=y^{i}=\left(1-\gamma_{D}^{i}\right)\left[w^{i} \bar{e}^{i}+s_{L}^{i} \sum_{j \in J} p_{L, j} \bar{L}_{j}\right]$ for $i \in\{m, h, s\}$.

From profit maximization in industry $H, \quad K_{H, j}=\beta_{K, H} p_{L, j} \bar{L}_{j} /\left(1-\beta_{K, H}\right) \bar{p}_{K}, \quad p_{H, j}=$ $\left(\bar{p}_{K} / \beta_{K, H}\right)^{\beta_{K, H}}\left[p_{L, j} /\left(1-\beta_{K, H}\right)\right]^{1-\beta_{K, H}}$, and $p_{H, j} Q_{H, j}=p_{L, j} \bar{L}_{j} /\left(1-\beta_{K, H}\right)$, where $\bar{p}_{K}>0$. Since $p_{H, j}=p_{H}$ for $j \in J$, it must be the case that $p_{L, j}=p_{L}$ for $j \in J$. Let $Q_{H}=\sum_{j \in J} Q_{H, j}$. Then, the market clearing condition in the housing market is $p_{H} Q_{H}=\alpha \sum_{i \in I} y^{i} N^{i}$. Introducing housing supply and the income of each group into this market clearing condition, the equilibrium price of land is

$$
p_{L}=\alpha\left(1-\beta_{K, H}\right) \beta_{U, C} \bar{p}_{C} Q_{C}(\bar{L})^{-1}\left[\frac{\beta_{U, C} \sum_{i=m, h} \eta_{D}^{i} n^{i}+\eta_{D}^{s}\left(1-\beta_{U, C}\right)}{\beta_{U, C}\left[1-\alpha\left(1-\beta_{K, H}\right) \eta_{D}^{s}\right]}\right],
$$

where $n^{i}=\bar{e}^{i} N^{i}\left(\sum_{i=m, h} \bar{e}^{i} N^{i}\right)^{-1}$ for $i=m, h$ and $\eta_{D}^{i}=\left(1-\gamma_{D}^{i}+\delta \gamma_{D}^{i}\right)$. Equilibrium housing prices are $p_{H_{u n}}=\left(\bar{p}_{K} / \beta_{K, H}\right)^{\beta_{K, H}}\left[p_{L} /\left(1-\beta_{K, H}\right)\right]^{1-\beta_{K, H}}$.

Finally, it still has to be checked that no agent has an incentive to change occupation. The indirect utility of an agent in group $i$ who selects $\left(o^{i}, r^{i}\right)$ is $v^{i}\left(o^{i}, r^{i}\right)=y^{i}\left(o^{i}, r^{i}\right) /\left(p_{H, r^{i}}\right)^{\alpha}\left(\bar{p}_{C}\right)^{1-\alpha}$. Therefore, we have:

Group $l$. Agents in group $l$ prefer to be criminals provided that $y^{l}>\left(1-\gamma_{D}^{l}\right) w^{u} \bar{e}^{l}$ or, which is equivalent, $\delta\left(N^{l}\right)^{-1} \sum_{i=m, h} \frac{\gamma_{D}^{s}+\beta_{U, C}\left[1-\alpha\left(1-\beta_{K, H}\right)\right]\left(\gamma_{D}^{i}-\gamma_{D}^{s}\right)}{\beta_{U, C}\left[1-\alpha\left(1-\beta_{K, H}\right) \eta_{D}^{s}\right]} \bar{e}^{i} N^{i}>\left(1-\gamma_{D}^{l}\right) \bar{e}^{l}$.

Group $i \in\{m, h\}$. Agents in group $i \in\{m, h\}$ prefer to be workers if $y^{l}<\left(1-\gamma_{D}^{i}\right) w^{u} \bar{e}^{i}$ or, which is equivalent, $\delta\left(N^{l}\right)^{-1} \sum_{i=m, h} \frac{\gamma_{D}^{s}+\beta_{U, C}\left[1-\alpha\left(1-\beta_{K, H}\right)\right]\left(\gamma_{D}^{i}-\gamma_{D}^{s}\right)}{\beta_{U, C}\left[1-\alpha\left(1-\beta_{K, H}\right) \eta_{D}^{s}\right]} \bar{e}^{i} N^{i}<\left(1-\gamma_{D}^{i}\right) \bar{e}^{i}$.

Group $s$. Agents in group $s$ prefer to be workers if $y^{l}<\left(1-\gamma_{D}^{s}\right) w^{s} \bar{e}^{s}+\left(N^{s}\right)^{-1} p_{L} \bar{L}$.

Employing Assumption 1, these conditions simplify to $\left(1-\gamma_{D}^{l}\right) \bar{e}^{l}<$ $\delta\left(N^{l}\right)^{-1} \sum_{i=m, h} \frac{\gamma_{D}^{s}+\beta_{U, C}\left[1-\alpha\left(1-\beta_{K, H}\right)\right]\left(\gamma_{D}^{i}-\gamma_{D}^{s}\right)}{\beta_{U, C}\left[1-\alpha\left(1-\beta_{K, H}\right) \eta_{D}^{s}\right]} \bar{e}^{i} N^{i}<\left(1-\gamma_{D}^{m}\right) \bar{e}^{m}$. This completes the proof of Lemma 2.

Proof of Proposition 1. Under the assumptions and conditions in Lemmas 1 and 2.

Part 1 (crime). Let $C R_{C}$ and $C R_{D}$ denote the value of stolen goods under concentrated and dispersed protection, respectively. Then, $C R_{C}=\sum_{i=m, h} \gamma^{i} N^{i} \bar{e}^{i}$ and $C R_{D}=\sum_{i=m, h} \tilde{\gamma}_{D}^{i} \bar{e}^{i} N^{i}$, where $\tilde{\gamma}_{D}^{i}=\frac{\gamma_{D}^{s}+\beta_{U, C}\left[1-\alpha\left(1-\beta_{K, H}\right)\right]\left(\gamma_{D}^{i}-\gamma_{D}^{s}\right)}{\beta_{U, C}\left[1-\alpha\left(1-\beta_{K, H}\right) \eta_{D}^{s}\right]}$. Then, $C R_{C}<C R_{D}$ if and only if $\sum_{i=m, h}\left(\gamma^{i}-\tilde{\gamma}_{D}^{i}\right) N^{i} \bar{e}^{i}<0$ or, 
which is equivalent,

$$
\sum_{i=m, h} \gamma^{i} n^{i}<\frac{\left[\frac{1-\beta_{U, C}}{\beta_{U, C}}+\alpha\left(1-\beta_{K, H}\right)\right](1-p) \gamma^{s}}{p\left[1-\alpha\left(1-\beta_{K, H}\right)\right]+(1-p) \alpha\left(1-\beta_{K, H}\right)(1-\delta) \gamma^{s}} .
$$

The left-hand side is increasing in $\gamma^{m}$ and $\gamma^{h}$, while the right-hand side does not depend on $\gamma^{m}$ or $\gamma^{h}$. The right-hand side is decreasing in $\beta_{U, C}$ and $p$ and increasing in $\gamma^{s}$, while the left-hand side does not depend on either of these variables. Therefore, the inequality is more likely to hold when $\gamma^{m}, \gamma^{h}, \beta_{U, C}$, and $p$ are low and when $\gamma^{s}$ is high.

Part 2 (aggregate income). Let $Y_{C}$ and $Y_{D}$ denote the aggregate income under concentrated and dispersed protection, respectively. Then, $Y_{C}=\beta_{U, C} \bar{p}_{C} Q_{C} \sum_{i=m, h} \tilde{\eta}_{C}^{i} n^{i}$ and $Y_{D}=$ $\beta_{U, C} \bar{p}_{C} Q_{C} \sum_{i=m, h} \tilde{\eta}_{D}^{i} n^{i}$, where $\tilde{\eta}_{C}^{i}=\frac{\beta_{U, C} \eta_{C}^{i}+\left(1-\beta_{U, C}\right)}{\beta_{U, C}\left[1-\alpha\left(1-\beta_{K, H}\right)\right]}$ and $\tilde{\eta}_{D}^{i}=\frac{\beta_{U, C} \eta_{D}^{i}+\left(1-\beta_{U, C}\right) \eta_{D}^{s}}{\beta_{U, C}\left[1-\alpha\left(1-\beta_{K, H}\right) \eta_{D}^{s}\right]}$. Then, $Y_{C}>Y_{D}$ if and only if $\sum_{i=m, h}\left(\tilde{\eta}_{C}^{i}-\tilde{\eta}_{D}^{i}\right) n^{i}>0$ or, which is equivalent,

$$
\beta_{U, C}<\frac{\gamma^{s}}{\left[1-\alpha\left(1-\beta_{K, H}\right)\right] \gamma^{s}+\left[\frac{p}{1-p}+\alpha\left(1-\beta_{K, H}\right)(1-\delta) \gamma^{s}\right] \sum_{i=m, h} \gamma^{i} n^{i}} .
$$

The right-hand side is decreasing in $\gamma^{m}, \gamma^{h}$ and $p$ and increasing in $\gamma^{s}$. Therefore, the inequality is more likely to hold when $\gamma^{m}, \gamma^{h}, \beta_{U, C}$, and $p$ are low and when $\gamma^{s}$ is high.

Part 3 (land prices). Land prices under concentrated protection are $p_{L_{u n}}=$ $\alpha\left(1-\beta_{K, H}\right) \beta_{U, C} \bar{p}_{C} Q_{C}\left(\bar{L}_{u n}\right)^{-1} \sum_{i=m, h} \eta_{C}^{i} n^{i}$ and $p_{L_{p}}=\alpha\left(1-\beta_{K, H}\right) \beta_{U, C} \bar{p}_{C} Q_{C}\left(\bar{L}_{p}\right)^{-1} \sum_{i=m, h} \hat{\eta}_{C}^{i} n^{i}$, where $\hat{\eta}_{C}^{i}=\frac{\beta_{U, C} \alpha\left(1-\beta_{K, H}\right) \eta_{C}^{i}+\left(1-\beta_{U, C}\right)}{\beta_{U, C}\left[1-\alpha\left(1-\beta_{K, H}\right)\right]}$. The price of land under dispersed protection is $p_{L}=$ $\alpha\left(1-\beta_{K, H}\right) \beta_{U, C} \bar{p}_{C} Q_{C}(\bar{L})^{-1} \sum_{i=m, h} \tilde{\eta}_{D}^{i} n^{i}$, where $\tilde{\eta}_{D}^{i}=\frac{\beta_{U, C} \eta_{D}^{i}+\left(1-\beta_{U, C}\right) \eta_{D}^{s}}{\beta_{U, C}\left[1-\alpha\left(1-\beta_{K, H}\right) \eta_{D}^{s}\right]}$. Therefore, $p_{L_{u n}}<p_{L}<$ $p_{L_{p}}$ if and only if

$$
\left(\bar{L}_{u n}\right)^{-1} \sum_{i=m, h} \eta_{C}^{i} n^{i}<(\bar{L})^{-1} \sum_{i=m, h} \tilde{\eta}_{D}^{i} n^{i}<\left(\bar{L}_{p}\right)^{-1} \sum_{i=m, h} \hat{\eta}_{C}^{i} n^{i}
$$

These inequalities are equivalent to $\frac{\bar{L}_{u n}}{\bar{L}}>\max \left\{\kappa_{1}, \kappa_{2}\right\}$, where $\kappa_{1}=\left(\sum_{i=m, h} \tilde{\eta}_{D}^{i} n^{i}\right)^{-1}\left(\sum_{i=m, h} \eta_{C}^{i} n^{i}\right)$ and $\kappa_{2}=\left(\sum_{i=m, h} \tilde{\eta}_{D}^{i} n^{i}\right)^{-1}\left[\sum_{i=m, h}\left(\tilde{\eta}_{D}^{i}-\hat{\eta}_{C}^{i}\right) n^{i}\right]$. Note that

$$
\frac{\partial \kappa_{1}}{\partial\left(\frac{1-\beta_{U, C}}{\beta_{U, C}}\right)}=\frac{-\eta_{D}^{s} \kappa_{1}}{\left[1-\alpha\left(1-\beta_{K, H}\right) \eta_{D}^{s}\right]\left(\sum_{i=m, h} \tilde{\eta}_{D}^{i} n^{i}\right)}<0
$$


and

$$
\begin{aligned}
\frac{\partial \kappa_{2}}{\partial\left(\frac{1-\beta_{U, C}}{\beta_{U, C}}\right)}= & {\left[\frac{\eta_{D}^{s}}{1-\alpha\left(1-\beta_{K, H}\right) \eta_{D}^{s}}-\frac{1}{1-\alpha\left(1-\beta_{K, H}\right)}\right] \frac{1}{\sum_{i=m, h} \tilde{\eta}_{D}^{i} n^{i}}+} \\
& -\frac{\eta_{D}^{s}}{\left[1-\alpha\left(1-\beta_{K, H}\right) \eta_{D}^{s}\right]} \frac{\kappa_{2}}{\sum_{i=m, h} \tilde{\eta}_{D}^{i} n^{i}}
\end{aligned}
$$

If $\kappa_{2}<0$, then it is not relevant because $\kappa_{1}>0$. If $\kappa_{2}>0$, then it may be relevant. Since $\eta_{D}^{s}<1$, then $\left[\frac{\eta_{D}^{s}}{1-\alpha\left(1-\beta_{K, H}\right) \eta_{D}^{s}}-\frac{1}{1-\alpha\left(1-\beta_{K, H}\right)}\right]<0$ and, hence, $\partial \kappa_{2} / \partial\left(\frac{1-\beta_{U, C}}{\beta_{U, C}}\right)<0$. Thus, $\bar{L}_{u n} / \bar{L}>\max \left\{\kappa_{1}, \kappa_{2}\right\}$ is more likely to hold when $\beta_{U, C}$ is lower. This completes the proof of Proposition 1.

Proof of Proposition 2. Under the assumptions and conditions in Lemmas 1 and 2.

Part 1. Under concentrated protection, the utility of an agent in group $i$ is $v_{C}^{i}=$ $v\left(N^{i}\right)^{-1}\left(Q_{C}\right)^{1-\alpha\left(1-\beta_{K, H}\right)}\left(\bar{L}_{r^{i}}\right)^{\alpha\left(1-\beta_{K, H}\right)} \Gamma_{C}^{i}$, where

$$
\begin{aligned}
& v=\left[\frac{\left(\alpha \beta_{K, H}\right)^{\alpha \beta_{K, H}}\left(\beta_{U, C}\right)^{1-\alpha\left(1-\beta_{K, H}\right)}}{(\alpha)^{\alpha}}\right]\left(\frac{\bar{p}_{C}}{\bar{p}_{K}}\right)^{\alpha \beta_{K, H}} \\
& \Gamma_{C}^{l}=\frac{\delta \sum_{i=m, h} \gamma_{C}^{i} n^{i}}{\left[\sum_{i=m, h} \eta_{C}^{i} n^{i}\right]^{\alpha\left(1-\beta_{K, H}\right)}}, \Gamma_{C}^{m}=\frac{\left(1-\gamma_{C}^{m}\right) n^{m}}{\left[\sum_{i=m, h} \eta_{C}^{i} n^{i}\right]^{\alpha\left(1-\beta_{K, H}\right)}}, \\
& \Gamma_{C}^{h}=\frac{\left(1-\gamma_{C}^{h}\right) n^{h}}{\left[\sum_{i=m, h} \eta_{C}^{i} n^{i}\right]^{\alpha\left(1-\beta_{K, H}\right)}}, \Gamma_{C}^{s}=\left[\sum_{i=m, h} \hat{\eta}_{C}^{i} n^{i}\right]^{1-\alpha\left(1-\beta_{K, H}\right)} .
\end{aligned}
$$

Under dispersed protection, the utility of an agent in group $i$ is $v_{D}^{i}=$ $\left(N^{i}\right)^{-1} v\left(Q_{C}\right)^{1-\alpha\left(1-\beta_{K, H}\right)}(\bar{L})^{\alpha\left(1-\beta_{K, H}\right)} \Gamma_{D}^{i}$, where

$$
\begin{aligned}
\Gamma_{D}^{l} & =\frac{\delta \sum_{i=m, h} \tilde{\gamma}_{D}^{i} n^{i}}{\left[\sum_{i=h, m} \tilde{\eta}_{D}^{i} n^{i}\right]^{\alpha\left(1-\beta_{K, H}\right)}}, \Gamma_{D}^{m}=\frac{\left(1-\gamma_{D}^{m}\right) n^{m}}{\left[\sum_{i=h, m} \tilde{\eta}_{D}^{i} n^{i}\right]^{\alpha\left(1-\beta_{K, H}\right)}} \\
\Gamma_{D}^{h} & =\frac{\left(1-\gamma_{D}^{h}\right) n^{h}}{\left[\sum_{i=h, m} \tilde{\eta}_{D}^{i} n^{i}\right]^{\alpha\left(1-\beta_{K, H}\right)}}, \Gamma_{D}^{s}=\frac{\left(1-\gamma_{D}^{s}\right) \sum_{i=h, m} \hat{\eta}_{D}^{i} n^{i}}{\left[\sum_{i=h, m} \tilde{\eta}_{D}^{i} n^{i}\right]^{\alpha\left(1-\beta_{K, H}\right)}}
\end{aligned}
$$

Then, $v_{D}^{l}>v_{C}^{l}$ if and only if $\bar{L}_{u n} / \bar{L}<\left(\Gamma_{D}^{l} / \Gamma_{C}^{l}\right)^{\frac{1}{\alpha\left(1-\beta_{K, H}\right)}}, v_{D}^{i}>v_{C}^{i}$ for $i=m, h$ if and only if $\bar{L}_{u n} / \bar{L}<\left(\Gamma_{D}^{i} / \Gamma_{C}^{i}\right)^{\frac{1}{\alpha\left(1-\beta_{K, H}\right)}}$, and $v_{C}^{s}>v_{D}^{s}$ if and only if $\bar{L}_{u n} / \bar{L}<1-\left(\Gamma_{D}^{s} / \Gamma_{C}^{s}\right)^{\frac{1}{\alpha\left(1-\beta_{K, H}\right)}}$.

Part 2. Under concentrated protection, the welfare of unskilled agents is given by

$$
\begin{aligned}
W_{C}^{u} & =\sum_{i=l, m, h} N^{i} v_{C}^{i}=v\left(Q_{C}\right)^{1-\alpha\left(1-\beta_{K, H}\right)}\left(\bar{L}_{u n}\right)^{\alpha\left(1-\beta_{K, H}\right)} \sum_{i=l, m, h} \Gamma_{C}^{i} \\
& =v\left(Q_{C}\right)^{1-\alpha\left(1-\beta_{K, H}\right)}\left(\bar{L}_{u n}\right)^{\alpha\left(1-\beta_{K, H}\right)} \Gamma_{C}^{u},
\end{aligned}
$$


where $\Gamma_{C}^{u}=\left[\sum_{i=m, h} \eta_{C}^{i} n^{i}\right]^{1-\alpha\left(1-\beta_{K, H}\right)}$. Under dispersed protection, the welfare of unskilled agents is given by

$$
\begin{aligned}
W_{D}^{u} & =\sum_{i=l, m, h} N^{i} v_{D}^{i}=v\left(Q_{C}\right)^{1-\alpha\left(1-\beta_{K, H}\right)}\left(\bar{L}_{1}+\bar{L}_{2}\right)^{\alpha\left(1-\beta_{K, H}\right)} \sum_{i=l, m, h} \Gamma_{D}^{i} \\
& =v\left(Q_{C}\right)^{1-\alpha\left(1-\beta_{K, H}\right)}(\bar{L})^{\alpha\left(1-\beta_{K, H}\right)} \Gamma_{D}^{u},
\end{aligned}
$$

where $\Gamma_{D}^{u}=\left[\sum_{i=h, m} \tilde{\eta}_{D}^{i} n^{i}\right]^{-\alpha\left(1-\beta_{K, H}\right)}\left[\sum_{i=m, h}\left(\eta_{D}^{i}+\delta \gamma_{D}^{s} \frac{\beta_{U, C} \alpha\left(1-\beta_{K, H}\right) \eta_{D}^{i}+\left(1-\beta_{U, C}\right)}{\beta_{U, C}\left[1-\alpha\left(1-\beta_{K, H}\right) \eta_{D}^{s}\right]}\right) n^{i}\right]$. Therefore $W_{D}^{u}>W_{C}^{u}$ if and only if $(\bar{L})^{\alpha\left(1-\beta_{K, H}\right)} \Gamma_{D}^{u}>\left(\bar{L}_{u n}\right)^{\alpha\left(1-\beta_{K, H}\right)} \Gamma_{C}^{u}$. Since $\eta_{D}^{i}>\eta_{C}^{i}$, we have $\Gamma_{D}^{u}>\Gamma_{C}^{u}$ and, hence, this inequality always holds.

Part 3. Aggregate welfare under concentrated protection is given by

$$
W_{C}=v\left(Q_{C}\right)^{1-\alpha\left(1-\beta_{K, H}\right)}\left[\Gamma_{C}^{u}\left(\bar{L}_{u n}\right)^{\alpha\left(1-\beta_{K, H}\right)}+\Gamma_{C}^{s}\left(\bar{L}_{p}\right)^{\alpha\left(1-\beta_{K, H}\right)}\right],
$$

where $\Gamma_{C}^{u}=\left[\sum_{i=m, h} \eta_{C}^{i} n^{i}\right]^{1-\alpha\left(1-\beta_{K, H}\right)}$ and $\Gamma_{C}^{s}=\left[\sum_{i=m, h} \hat{\eta}_{C}^{i} n^{i}\right]^{1-\alpha\left(1-\beta_{K, H}\right)}$. Analogously, aggregate welfare under dispersed protection is given by

$$
W_{D}=v\left(Q_{C}\right)^{1-\alpha\left(1-\beta_{K, H}\right)}\left(\bar{L}_{1}+\bar{L}_{2}\right)^{\alpha\left(1-\beta_{K, H}\right)} \Gamma_{D},
$$

where $\Gamma_{D}=\left[\sum_{i=m, h} \tilde{\eta}_{D}^{i} n^{i}\right]^{1-\alpha\left(1-\beta_{K, H}\right)}$. Therefore, $W_{C}>W_{D}$, if and only if

$$
\Gamma_{C}=\Gamma_{C}^{u}\left(\frac{\bar{L}_{u n}}{\bar{L}}\right)^{\alpha\left(1-\beta_{K, H}\right)}+\Gamma_{C}^{s}\left(\frac{\bar{L}_{p}}{\bar{L}}\right)^{\alpha\left(1-\beta_{K, H}\right)}>\Gamma_{D} .
$$

Next, we prove that there exists a threshold for $\left(1-\beta_{U, C}\right) / \beta_{U, C}$ such that $W_{C}<W_{D}$ if and only if $\left(1-\beta_{U, C}\right) / \beta_{U, C}$ is below it.

Step 1. First, we prove that equations (3), (4) and (8) impose an upper bound on $\left(1-\beta_{U, C}\right) / \beta_{U, C}$.

From equation (3) we have:

$$
\frac{\left(1-\gamma^{l}\right) \bar{e}^{l} N^{l}}{\sum_{i=m, h} \gamma^{i} N^{i} \bar{e}^{i}}<\delta<\frac{\left(1-\gamma^{m}\right) \bar{e}^{m} N^{l}}{\sum_{i=m, h} \gamma^{i} N^{i} \bar{e}^{i}} .
$$

From equation (4) we have:

$$
\begin{aligned}
& \left(\frac{1-\beta_{U, C}}{\beta_{U, C}}\right)>\left[\frac{\left[1-\alpha\left(1-\beta_{K, H}\right)\right] \bar{L}_{p}}{\left(1-\gamma^{h}\right)^{\frac{1}{\alpha\left(1-\beta_{K, H}\right)}}}-\alpha\left(1-\beta_{K, H}\right) \bar{L}_{u n}\right]\left[\frac{\sum_{i=m, h} \eta_{C}^{i} n^{i}}{\sum_{i=m, h} n^{i}}\right], \\
& \left(\frac{1-\beta_{U, C}}{\beta_{U, C}}\right)<B_{1}=\left[\frac{\left[1-\alpha\left(1-\beta_{K, H}\right)\right] \bar{L}_{p}}{\left(1-\gamma^{s}\right)^{\frac{\alpha\left(1-\beta_{K, H}\right)}{1}}}-\alpha\left(1-\beta_{K, H}\right) \bar{L}_{u n}\right]\left[\frac{\sum_{i=m, h} \eta_{C}^{i} n^{i}}{\sum_{i=m, h} n^{i}}\right] \text {. }
\end{aligned}
$$


From equation (8) we have:

$$
\begin{aligned}
\left(\frac{1-\beta_{U, C}}{\beta_{U, C}}\right)> & \frac{\left[1-\alpha\left(1-\beta_{K, H}\right) \eta_{D}^{s}\right]\left(1-\gamma_{D}^{l}\right) \bar{e}^{l} N^{l}}{\delta \gamma_{D}^{s} \sum_{i=m, h} \bar{e}^{i} N^{i}}+ \\
& -\frac{\sum_{i=m, h}\left\{\left[1-\alpha\left(1-\beta_{K, H}\right)\right] \gamma_{D}^{i}+\alpha\left(1-\beta_{K, H}\right) \gamma_{D}^{s}\right\} \bar{e}^{i} N^{i}}{\gamma_{D}^{s} \sum_{i=m, h} \bar{e}^{i} N^{i}} \\
\left(\frac{1-\beta_{U, C}}{\beta_{U, C}}\right)< & B_{2}=\frac{\left[1-\alpha\left(1-\beta_{K, H}\right) \eta_{D}^{s}\right]\left(1-\gamma_{D}^{m}\right) \bar{e}^{m} N^{l}}{\delta \gamma_{D}^{s} \sum_{i=m, h} \bar{e}^{i} N^{i}}+ \\
& -\frac{\sum_{i=m, h}\left\{\left[1-\alpha\left(1-\beta_{K, H}\right)\right] \gamma_{D}^{i}+\alpha\left(1-\beta_{K, H}\right) \gamma_{D}^{s}\right\} \bar{e}^{i} N^{i}}{\gamma_{D}^{s} \sum_{i=m, h} \bar{e}^{i} N^{i}} .
\end{aligned}
$$

Define:

$$
\begin{aligned}
\delta_{L} & =\max \left\{\frac{\left[1-\alpha\left(1-\beta_{K, H}\right) \eta_{D}^{s}\right]\left(1-\gamma_{D}^{l}\right) \bar{e}^{l} N^{l}}{\sum_{i=m, h}\left\{\left[1-\alpha\left(1-\beta_{K, H}\right)\right] \gamma_{D}^{i}+\alpha\left(1-\beta_{K, H}\right) \gamma_{D}^{s}\right\} \bar{e}^{i} N^{i}}, \frac{\left(1-\gamma^{l}\right) \bar{e}^{l} N^{l}}{\sum_{i=m, h} \gamma^{i} N^{i} \bar{e}^{i}}\right\} \\
\delta_{H} & =\min \left\{\frac{\left[1-\alpha\left(1-\beta_{K, H}\right) \eta_{D}^{s}\right]\left(1-\gamma_{D}^{m}\right) \bar{e}^{m} N^{l}}{\sum_{i=m, h}\left\{\left[1-\alpha\left(1-\beta_{K, H}\right)\right] \gamma_{D}^{i}+\alpha\left(1-\beta_{K, H}\right) \gamma_{D}^{s}\right\} \bar{e}^{i} N^{i}}, \frac{\left(1-\gamma^{m}\right) \bar{e}^{m} N^{l}}{\sum_{i=m, h} \gamma^{i} N^{i} \bar{e}^{i}}\right\} \\
\lambda_{L} & =\frac{\left[1-\alpha\left(1-\beta_{K, H}\right)\right]}{\left(1-\gamma^{h}\right)^{\frac{1}{\alpha\left(1-\beta_{K, H}\right)}} \alpha\left(1-\beta_{K, H}\right)} \\
\lambda_{H}^{1} & =\frac{\left[1-\alpha\left(1-\beta_{K, H}\right)\right]}{\left(1-\gamma^{s}\right)^{\frac{1}{\alpha\left(1-\beta_{K, H}\right)}} \alpha\left(1-\beta_{K, H}\right)}
\end{aligned}
$$

Assume that $\delta_{L}<\delta<\delta_{H}$ and $\lambda_{L}<\bar{L}_{u n} / \bar{L}_{p}<\lambda_{H}^{1}$. Then, equations (3), (4) and (8) only impose a strictly positive upper bound on $\left(1-\beta_{U, C}\right) / \beta_{U, C}$, which is given either by $B_{1}$ or $B_{2}$. Denote such upper bound $\left(1-\beta_{U, C}^{\min }\right) / \beta_{U, C}^{\min }$.

Step 2. We study how $\Gamma_{C}$ and $\Gamma_{D}$ vary with $\left(1-\beta_{U, C}\right) / \beta_{U, C}$. $\Gamma_{C}$ is given by:

$$
\begin{aligned}
\Gamma_{C}= & {\left[\sum_{i=m, h} \eta_{C}^{i} n^{i}\right]^{1-\alpha\left(1-\beta_{K, H}\right)}\left(\frac{\bar{L}_{u n}}{\bar{L}}\right)^{\alpha\left(1-\beta_{K, H}\right)} } \\
& +\left\{\frac{\sum_{i=m, h}\left[\alpha\left(1-\beta_{K, H}\right) \eta_{C}^{i}+\left(\frac{1-\beta_{U, C}}{\beta_{U, C}}\right)\right] n^{i}}{1-\alpha\left(1-\beta_{K, H}\right)}\right\}^{1-\alpha\left(1-\beta_{K, H}\right)}\left(\frac{\bar{L}_{p}}{\bar{L}}\right)^{\alpha\left(1-\beta_{K, H}\right)}
\end{aligned}
$$


- $\Gamma_{C}$ is strictly increasing and strictly concave in $\frac{1-\beta_{U, C}}{\beta_{U, C}}$ :

$$
\begin{aligned}
\frac{\partial \Gamma_{C}}{\partial\left(\frac{1-\beta_{U, C}}{\beta_{U, C}}\right)}= & \frac{\left[1-\alpha\left(1-\beta_{K, H}\right)\right] \sum_{i=m, h} n^{i} \Gamma_{C}^{s}\left(\frac{\bar{L}_{p}}{\bar{L}}\right)^{\alpha\left(1-\beta_{K, H}\right)}}{\sum_{i=m, h}\left[\alpha\left(1-\beta_{K, H}\right) \eta_{C}^{i}+\left(\frac{1-\beta_{U, C}}{\beta_{U, C}}\right)\right] n^{i}}>0 \\
\frac{\partial^{2} \Gamma_{C}}{\partial\left(\frac{1-\beta_{U, C}}{\beta_{U, C}}\right)^{2}}= & -\frac{\left[1-\alpha\left(1-\beta_{K, H}\right)\right] \alpha\left(1-\beta_{K, H}\right)\left[\sum_{i=m, h} n^{i}\right]^{2} \Gamma_{C}^{s}\left(\frac{\bar{L}_{p}}{\bar{L}}\right)^{\alpha\left(1-\beta_{K, H}\right)}}{\left\{\sum_{i=m, h}\left[\alpha\left(1-\beta_{K, H}\right) \eta_{C}^{i}+\left(\frac{1-\beta_{U, C}}{\beta_{U, C}}\right)\right] n^{i}\right\}^{2}}<0
\end{aligned}
$$

- $\lim _{\left(1-\beta_{U, C}\right) / \beta_{U, C} \rightarrow 0} \Gamma_{C} \quad=\quad\left[\frac{\sum_{i=m, h} \alpha\left(1-\beta_{K, H}\right) \eta_{C}^{i} n^{i}}{1-\alpha\left(1-\beta_{K, H}\right)}\right]^{1-\alpha\left(1-\beta_{K, H}\right)}\left(\bar{L}_{p} / \bar{L}\right)^{\alpha\left(1-\beta_{K, H}\right)} \quad$ and $\lim _{\left(1-\beta_{U, C}\right) / \beta_{U, C} \rightarrow 0} \Gamma_{C}=\infty$.

$\Gamma_{D}$ is given by:

$$
\Gamma_{D}=\left\{\frac{\sum_{i=m, h}\left[\eta_{D}^{i}+\left(\frac{1-\beta_{U, C}}{\beta_{U, C}}\right) \eta_{D}^{s}\right] n^{i}}{1-\alpha\left(1-\beta_{K, H}\right) \eta_{D}^{s}}\right\}^{1-\alpha\left(1-\beta_{K, H}\right)}
$$

- $\Gamma_{D}$ is strictly increasing and strictly concave in $\left(1-\beta_{U, C}\right) / \beta_{U, C}$ :

$$
\begin{aligned}
\frac{\partial \Gamma_{D}}{\partial\left(\frac{1-\beta_{U, C}}{\beta_{U, C}}\right)} & =\frac{\eta_{D}^{s}\left(\sum_{i=m, h} n^{i}\right)\left\{\sum_{i=m, h}\left[\eta_{D}^{i}+\left(\frac{1-\beta_{U, C}}{\beta_{U, C}}\right) \eta_{D}^{s}\right] n^{i}\right\}^{-\alpha\left(1-\beta_{K, H}\right)}}{\left[1-\alpha\left(1-\beta_{K, H}\right)\right]^{-1}\left[1-\alpha\left(1-\beta_{K, H}\right) \eta_{D}^{s}\right]^{1-\alpha\left(1-\beta_{K, H}\right)}}>0 \\
\frac{\partial^{2} \Gamma_{D}}{\partial\left(\frac{1-\beta_{U, C}}{\beta_{U, C}}\right)^{2}}<0 & <
\end{aligned}
$$

- $\lim _{\left(1-\beta_{U, C}\right) / \beta_{U, C} \rightarrow 0} \Gamma_{D}=\left[\frac{\sum_{i=m, h} \eta_{D}^{i} n^{i}}{\left[1-\alpha\left(1-\beta_{K, H}\right) \eta_{D}^{s}\right]}\right]^{1-\alpha\left(1-\beta_{K, H}\right)}$ and $\lim _{\left(1-\beta_{U, C}\right) / \beta_{U, C} \rightarrow 0} \Gamma_{D}=\infty$.

Step 3. We compare $\Gamma_{C}$ and $\Gamma_{D}$.

- Note that $\lim _{\left(1-\beta_{U, C}\right) / \beta_{U, C} \rightarrow 0} \Gamma_{D}>\lim _{\left(1-\beta_{U, C}\right) / \beta_{U, C} \rightarrow 0} \Gamma_{C}$ if and only if

$$
\left[\frac{\sum_{i=m, h} \eta_{D}^{i} n^{i}}{\left[1-\alpha\left(1-\beta_{K, H}\right) \eta_{D}^{s}\right]}\right]^{1-\alpha\left(1-\beta_{K, H}\right)}>\left[\frac{\sum_{i=m, h} \alpha\left(1-\beta_{K, H}\right) \eta_{C}^{i} n^{i}}{1-\alpha\left(1-\beta_{K, H}\right)}\right]^{1-\alpha\left(1-\beta_{K, H}\right)}\left(\frac{\bar{L}_{p}}{\bar{L}}\right)^{\alpha\left(1-\beta_{K, H}\right)}
$$

Since $\eta_{D}^{i}>\eta_{C}^{i}$, this condition always holds. 
- $\partial \Gamma_{C} / \partial\left[\left(1-\beta_{U, C}\right) / \beta_{U, C}\right]>\partial \Gamma_{D} / \partial\left[\left(1-\beta_{U, C}\right) / \beta_{U, C}\right]$ if and only if

$$
\left\{\frac{1-\alpha\left(1-\beta_{K, H}\right) \eta_{D}^{s}}{\left[1-\alpha\left(1-\beta_{K, H}\right)\right] \eta_{D}^{s}}\right\}^{1-\alpha\left(1-\beta_{K, H}\right)}\left\{\frac{\bar{L}_{p} \sum_{i=m, h}\left[\frac{\eta_{D}^{i}}{\eta_{D}^{s}}+\left(\frac{1-\beta_{U, C}}{\beta_{U, C}}\right)\right] n^{i}}{\bar{L} \sum_{i=m, h}\left[\alpha\left(1-\beta_{K, H}\right) \eta_{C}^{i}+\left(\frac{1-\beta_{U, C}}{\beta_{U, C}}\right)\right] n^{i}}\right\}^{\alpha\left(1-\beta_{K, H}\right)}>1
$$

Since $\eta_{D}^{i}>\eta_{C}^{i}$, a sufficient condition for this inequality to be satisfied is

$$
\frac{\bar{L}_{u n}}{\bar{L}_{p}}<\lambda_{H}^{2}=\left[\frac{1-\alpha\left(1-\beta_{K, H}\right) \eta_{D}^{s}}{\eta_{D}^{s}-\alpha\left(1-\beta_{K, H}\right) \eta_{D}^{s}}\right]^{\frac{1-\alpha\left(1-\beta_{K, H}\right)}{\alpha\left(1-\beta_{K, H}\right)}}-1
$$

Let $\lambda_{H}=\min \left\{\lambda_{H}^{1}, \lambda_{H}^{2}\right\}$. Then, combining Steps 1, 2, and 3 we have proved that if $\delta_{L}<\delta<\delta_{H}$ and $\lambda_{L}<\frac{\bar{L}_{1}}{L_{2}}<\lambda_{H}$, then there exists $\bar{\beta}_{U, C}$ such that for all $\left(1-\beta_{U, C}\right) / \beta_{U, C}<\left(1-\bar{\beta}_{U, C}\right) / \bar{\beta}_{U, C}$ we have $\Gamma_{C}<\Gamma_{D}$ and for all $\left(1-\bar{\beta}_{U, C}\right) / \bar{\beta}_{U, C}<\left(1-\beta_{U, C}\right) / \beta_{U, C}$ we have $\Gamma_{C}>\Gamma_{D}$. Finally, Step 1 also implies that $\left(1-\beta_{U, C}\right) / \beta_{U, C} \in\left(0,\left(1-\beta_{U, C}^{\min }\right) / \beta_{U, C}^{\min }\right)$ and we have already proved that $\Gamma_{C}>\Gamma_{D}$ if and only if $W_{C}>W_{D}$. This completes the proof of Proposition 2 .

Compensations. Suppose that protection is concentrated and the government also set a tax/transfer $\bar{p}_{C} \tau_{f}^{i}$ on each agent in group $i \in I$. The government budget constraint is $\sum_{i \in I} \tau_{f}^{i} N^{i}=0$. Following the same procedure we used in the proof of Lemma 1 we have:

$$
\begin{aligned}
y^{l}\left(b_{h}, j\right) & =y^{l}=\delta\left(N^{l}\right)^{-1}\left[\beta_{U, C} \bar{p}_{C} Q_{C} \sum_{i=m, h} \gamma^{i} n^{i}+\sum_{i=m, h} \gamma^{i} \bar{p}_{C} \tau_{f}^{i} N^{i}\right]+\bar{p}_{C} \tau_{f}^{l} N^{l} \text { for } h, j \in J_{u n}, \\
y^{m}(w, j) & =y^{m}=\left(N^{m}\right)^{-1}\left(1-\gamma^{m}\right)\left[\beta_{U, C} n^{m} \bar{p}_{C} Q_{C}+\bar{p}_{C} \tau_{f}^{m} N^{m}\right] \text { for } j \in J_{u n}, \\
y^{h}(w, j) & =y^{h}=\left(N^{h}\right)^{-1}\left(1-\gamma^{h}\right)\left[\beta_{U, C} n^{h} \bar{p}_{C} Q_{C}+\bar{p}_{C} \tau_{f}^{h} N^{h}\right] \text { for } j \in J_{u n}, \\
y^{s}(w, j) & =y^{s}=\left(N^{s}\right)^{-1}\left[\left(1-\beta_{U, C}\right) \bar{p}_{C} Q_{C}+p_{L_{u n}} \bar{L}_{u n}+p_{L_{p}} \bar{L}_{p}+\bar{p}_{C} \tau_{f}^{s} N^{s}\right] \text { for } j \in J_{p},
\end{aligned}
$$

where $w^{u}=\bar{p}_{C} \beta_{U, C}\left(S_{C} / U_{C}\right)^{1-\beta_{U, C}}, w^{s}=\bar{p}_{C}\left(1-\beta_{U, C}\right)\left(U_{C} / S_{C}\right)^{\beta_{U, C}}, U_{C}=\sum_{i=m, h} N^{i} \bar{e}^{i}, S_{C}=N^{s} \bar{e}^{s}$ and $Q_{C}=\left(U_{C}\right)^{\beta_{U, C}}\left(S_{C}\right)^{1-\beta_{U, C}}$. Market clearing conditions in the housing markets are $p_{H_{u n}} Q_{H_{u n}}=$ $\alpha \sum_{i=l . m . h} y^{i} N^{i}$ and $p_{H_{p}} Q_{H_{p}}=\alpha y^{s} N^{s}$. Introducing housing supplies and the income of each group into these market clearing conditions, equilibrium land prices are

$$
\begin{aligned}
p_{L_{u n}} & =\alpha\left(1-\beta_{K, H}\right)\left(\bar{L}_{u n}\right)^{-1} \beta_{U, C} \bar{p}_{C} Q_{C}\left(\sum_{i=m, h} \eta_{C}^{i} n^{i}+T_{u n}\right) \\
p_{L_{p}} & =\alpha\left(1-\beta_{K, H}\right)\left(\bar{L}_{p}\right)^{-1} \beta_{U, C} \bar{p}_{C} Q_{C}\left(\sum_{i=m, h} \hat{\eta}_{C}^{i} n^{i}+T_{p}\right)
\end{aligned}
$$


where $T_{u n}=\left(\beta_{U, C} Q_{C}\right)^{-1} \sum_{i=m, h} \eta_{C}^{i} \tau_{f}^{i} N^{i}+\tau_{f}^{l} N^{l}$ and $T_{p}=\frac{\tau_{f}^{l} N^{l}+\tau_{f}^{s} N^{s}+\alpha\left(1-\beta_{K, H}\right) \sum_{i=m, h} \eta_{C}^{i} \tau_{f}^{i} N^{i}}{\left[1-\alpha\left(1-\beta_{K, H}\right)\right] \beta_{U, C} Q_{C}}$ are the new terms related to the tax/transfer program. Finally, we must check that no agent has an incentive to change his or her occupation and/or residence. Using the same procedure we employed in the proof of Lemma 1, we need to impose the following conditions:

$$
\begin{aligned}
\left(1-\gamma^{l}\right) \bar{e}^{l}-T^{l} & <\delta\left(N^{l}\right)^{-1} \sum_{i=m, h} \gamma^{i} N^{i} \bar{e}^{i}<\left(1-\gamma^{m}\right) \bar{e}^{m}-T^{m}, \\
\left(1-\gamma^{s}\right) & <\left[\frac{\bar{L}_{p}\left(\sum_{i=m, h} \eta_{C}^{i} n^{i}+T_{u n}\right)}{\bar{L}_{u n}\left(\sum_{i=m, h} \hat{\eta}_{C}^{i} n^{i}+T_{p}\right)}\right]^{\alpha\left(1-\beta_{K, H}\right)}<\left(1-\gamma^{h}\right),
\end{aligned}
$$

where $T^{m}=\left(\beta_{U, C} Q_{C}\right)^{-1} \sum_{i=m, h} \bar{e}^{i} N^{i}\left[\gamma^{m} \tau_{f}^{m}+\delta\left(N^{l}\right)^{-1} \sum_{i=m, h} \gamma^{i} \tau_{f}^{i} N^{i}\right] \quad$ and $\quad T^{l}=$ $\left(\beta_{U, C} Q_{C}\right)^{-1} \sum_{i=m, h} \bar{e}^{i} N^{i}\left[\gamma^{l} \tau_{f}^{l}+\delta\left(N^{l}\right)^{-1} \sum_{i=m, h} \gamma^{i} \tau_{f}^{i} N^{i}\right]$ are the new terms associated with the tax/transfer program.

The utility of an agent in group $i \in\{l, m, h\}$ is

$$
v_{C, \tau}^{i}=\left(N^{i}\right)^{-1} v\left(\frac{\bar{p}_{C}}{p_{K}}\right)^{\alpha \beta_{K, H}}\left(Q_{C}\right)^{1-\alpha\left(1-\beta_{K, H}\right)}\left(\bar{L}_{u n}\right)^{\alpha\left(1-\beta_{K, H}\right)} \Gamma_{C, \tau}^{i}
$$

where

$$
\begin{aligned}
& \Gamma_{C, \tau}^{l}=\left[\frac{\beta_{U, C} Q_{C} \delta \sum_{i=m, h} \gamma^{i} n^{i}+\delta \sum_{i=m, h} \gamma^{i} \tau_{f}^{i} N^{i}+\tau_{f}^{l} N^{l}}{\beta_{U, C} Q_{C}\left(\sum_{i=m, h} \eta_{C}^{i} n^{i}+T_{u n}\right)^{\alpha\left(1-\beta_{K, H}\right)}}\right], \\
& \Gamma_{C, \tau}^{m}=\left[\frac{\left(1-\gamma^{m}\right)\left(n^{m}+\frac{\tau_{f}^{m} N^{m}}{Q_{C}}\right)}{\left(\sum_{i=m, h} \eta_{C}^{i} n^{i}+T_{u n}\right)^{\alpha\left(1-\beta_{K, H}\right)}}\right] \\
& \Gamma_{C, \tau}^{h}=\left[\frac{\left(1-\gamma^{h}\right)\left(n^{h}+\frac{\tau_{f}^{h} N^{h}}{Q_{C}}\right)}{\left(\sum_{i=m, h} \eta_{C}^{i} n^{i}+T_{u n}\right)^{\alpha\left(1-\beta_{K, H}\right)}}\right] .
\end{aligned}
$$

Under dispersed protection with no tax/transfers, the utility of an agent in group $i \in\{l, m, h\}$ is 
$v_{D}^{i}=\left(N^{i}\right)^{-1} v\left(Q_{C}\right)^{1-\alpha\left(1-\beta_{K, H}\right)}(\bar{L})^{\alpha\left(1-\beta_{K, H}\right)} \Gamma_{D}^{i}$, where

$$
\begin{aligned}
\Gamma_{D}^{l} & =\frac{\delta \sum_{i=m, h} \tilde{\gamma}_{D}^{i} n^{i}}{\left[\sum_{i=h, m} \tilde{\eta}_{D}^{i} n^{i}\right]^{\alpha\left(1-\beta_{K, H}\right)}}, \Gamma_{D}^{m}=\frac{\left(1-\gamma_{D}^{m}\right) n^{m}}{\left[\sum_{i=h, m} \tilde{\eta}_{D}^{i} n^{i}\right]^{\alpha\left(1-\beta_{K, H}\right)}} \\
\Gamma_{D}^{h} & =\frac{\left(1-\gamma_{D}^{h}\right) n^{h}}{\left[\sum_{i=h, m} \tilde{\eta}_{D}^{i} n^{i}\right]^{\alpha\left(1-\beta_{K, H}\right)}}, \Gamma_{D}^{s}=\frac{\left(1-\gamma_{D}^{s}\right) \sum_{i=h, m} \hat{\eta}_{D}^{i} n^{i}}{\left[\sum_{i=h, m} \tilde{\eta}_{D}^{i} n^{i}\right]^{\alpha\left(1-\beta_{K, H}\right)}}
\end{aligned}
$$

Suppose that the government wants to make each agent in group $i \in\{l, m, h\}$ equally well off under concentrated and dispersed protection. Then, $\left(\tau_{f}^{l}, \tau_{f}^{m}, \tau_{f}^{h}\right)$ must be such that $v_{C, \tau}^{i}=v_{D}^{i}$ for $i \in\{l, m, h\}$, while $\tau_{f}^{s} N^{s}=-\sum_{i \in I} \tau_{f}^{i} N^{i}$. Thus, $\left(\tau_{f}^{l}, \tau_{f}^{m}, \tau_{f}^{h}\right)$ is the solution to the following system of equations:

$$
\begin{aligned}
& {\left[\frac{\sum_{i=m, h} \gamma^{i} n^{i}}{\sum_{i=m, h} \tilde{\gamma}_{D}^{i} n^{i}}+\frac{\delta \sum_{i=m, h} \gamma^{i} \tau_{f}^{i} N^{i}+\tau_{f}^{l} N^{l}}{\delta \beta_{U, C} Q_{C} \sum_{i=m, h} \tilde{\gamma}_{D}^{i} n^{i}}\right]\left[\frac{\Psi\left(\tau_{f}^{l}, \tau_{f}^{m}, \tau_{f}^{h}\right) \bar{L}_{u n}}{\bar{L}}\right]^{\alpha\left(1-\beta_{K, H}\right)}=1, } \\
& {\left[\left(\frac{1-\gamma^{m}}{1-\gamma_{D}^{m}}\right)\left(1+\frac{\tau_{f}^{m} N^{m}}{n^{m} Q_{C}}\right)\right]\left[\frac{\Psi\left(\tau_{f}^{l}, \tau_{f}^{m}, \tau_{f}^{h}\right) \bar{L}_{u n}}{\bar{L}}\right]^{\alpha\left(1-\beta_{K, H}\right)}=1, } \\
& {\left[\left(\frac{1-\gamma^{h}}{1-\gamma_{D}^{h}}\right)\left(1+\frac{\tau_{f}^{h} N^{h}}{n^{h} Q_{C}}\right)\right]\left[\frac{\Psi\left(\tau_{f}^{l}, \tau_{f}^{m}, \tau_{f}^{h}\right) \bar{L}_{u n}}{\bar{L}}\right]^{\alpha\left(1-\beta_{K, H}\right)}=1, }
\end{aligned}
$$

where $\Psi\left(\tau_{f}^{l}, \tau_{f}^{m}, \tau_{f}^{h}\right)=\frac{\beta_{U, C} Q_{C} \sum_{i=h, m} \tilde{\eta}_{D}^{i} n^{i}}{\beta_{U, C} Q_{C} \sum_{i=m, h} \eta_{C}^{i} n^{i}+\sum_{i=m, h} \eta_{C}^{i} \tau_{f}^{i} N^{i}+\tau_{f}^{l} N^{l}}$.

\section{Proof of Lemma 3.}

Part 1. Suppose that police protection is concentrated in neighborhoods $J_{p}$. Then, $p_{j}=1$ and $\gamma_{j}^{i}=0$ for $j \in J_{p}$ and $p_{j}=0$ and $\gamma_{j}^{i}=\gamma^{i}$ for $j \in J_{u n}$. For $j \in J_{u n}$ we have $A(0)=\infty$ and, hence, $p_{L^{p}, j}=\infty$. Thus, no agent can afford an area with private protection in $j \in J_{u n}$. For $j \in J_{p}$ we have $A\left(p_{j}\right)=A(1)$ and, hence, $p_{L^{p}, j}=p_{K} c_{\min } / A(1)+p_{L, j}$. However, no agent who decides to reside in $j \in J_{p}$ will be willing to pay for private protection because he or she is already fully protected.

Part 2. Suppose that police protection is evenly distributed in all neighborhoods. Then, $\gamma_{j}^{i}=$ $\gamma^{i}(1-p)$ for $j \in J$. In equilibrium the prices of land with and without security investment $\left(p_{L}, p_{L^{p}}\right)$ must be the same everywhere. Otherwise, agents will move to neighborhoods with lower land prices. Let $L_{p}$ be the supply of fully protected land. If $p_{L^{p}}<p_{K} c_{\min } / A(p)+p_{L}$, then $L_{p}=0$. If $p_{L^{p}}=p_{K} c_{\min } / A(p)+p_{L}$, 
developers will elastically supply fully protected areas each with $l_{\min }$ units of adjacent land. Next we consider two possible cases.

Part 2a. Suppose that agents in group $l$ are criminals, agents in groups $l, m$, and $h$ reside in areas without private security, and agents in group $s$ reside in fully protected areas with private security. Then, following the same procedure we use in the proof of Lemma 1, housing market clearing conditions become:

$$
\begin{aligned}
p_{L}\left(\bar{L}-L_{p}\right) & =\alpha\left(1-\beta_{K, H}\right) \beta_{U, C} \bar{p}_{C} Q_{C} \sum_{i=m, h} \eta_{D}^{i} n^{i} \\
p_{L^{p}} L_{p} & =\alpha\left(1-\beta_{K, H}\right) \beta_{U, C} \bar{p}_{C} Q_{C} \sum_{i=m, h} \hat{\eta}_{D}^{i} n^{i}
\end{aligned}
$$

where $\hat{\eta}_{D}^{i}=\frac{\beta_{U, C} \alpha\left(1-\beta_{K, H}\right) \eta_{D}^{i}+\left(1-\beta_{U, C}\right)}{\beta_{U, C}\left[1-\alpha\left(1-\beta_{K, H}\right)\right]}$. Contrary to Lemma $1, L_{p}$ is now an endogenous variable, but $p_{L}$ and $p_{L^{p}}$ are connected by $p_{L^{p}}=p_{K} c_{\min } / A(p)+p_{L}$. Employing these three expressions we obtain the following quadratic equation for $L_{p}$ :

$$
f\left(L_{p}\right)=a\left(L_{p}\right)^{2}+b L_{p}+c=0
$$

where

$$
\begin{aligned}
a & =\frac{p_{K} c_{\min }}{A(p)} \\
b & =-\alpha\left(1-\beta_{K, H}\right) \beta_{U, C} \bar{p}_{C} Q_{C} \sum_{i=m, h}\left(\eta_{D}^{i}+\hat{\eta}_{D}^{i}\right) n^{i}-\frac{p_{K} c_{\min }}{A(p)} \bar{L} \\
c & =\alpha\left(1-\beta_{K, H}\right) \beta_{U, C} \bar{p}_{C} Q_{C} \sum_{i=m, h} \hat{\eta}_{D}^{i} n^{i} \bar{L}
\end{aligned}
$$

Solving this quadratic equation we have $L_{p}=\frac{-b+-\sqrt{b^{2}-4 a c}}{2 a}$. Note that $b^{2}>4 a c, \frac{-b+-\sqrt{b^{2}-4 a c}}{2 a}>0$, but $\frac{-b+\sqrt{b^{2}-4 a c}}{2 a}>\bar{L}$. Thus, the relevant solution is

$$
L_{p}=\frac{-b-\sqrt{b^{2}-4 a c}}{2 a}
$$

Introducing $L_{p}$ into the housing market clearing conditions we obtain $p_{L}$ and $p_{L^{p}}$ :

$$
\begin{aligned}
p_{L} & =\alpha\left(1-\beta_{K, H}\right) \beta_{U, C} \bar{p}_{C} Q_{C}\left(\bar{L}-L_{p}\right)^{-1} \sum_{i=m, h} \eta_{D}^{i} n^{i} \\
p_{L^{p}} & =\alpha\left(1-\beta_{K, H}\right) \beta_{U, C} \bar{p}_{C} Q_{C}\left(L_{p}\right)^{-1} \sum_{i=m, h} \hat{\eta}_{D}^{i} n^{i} .
\end{aligned}
$$

For this solution to be an equilibrium we must verify that no agent prefers to change his or her occupational or residential choice. Regarding residence, there are no incentives to move if and only if 
$\left[1-\gamma^{s}(1-p)\right]<\left(p_{L} / p_{L^{p}}\right)^{\alpha\left(1-\beta_{K, H}\right)}<\left[1-\gamma^{h}(1-p)\right]$. After introducing $p_{L}$ and $p_{L^{p}}$ into these inequalities we obtain

$$
\bar{\lambda}^{s} \bar{L}<L_{p}=\frac{-b-\sqrt{b^{2}-4 a c}}{2 a}<\bar{\lambda}^{h} \bar{L},
$$

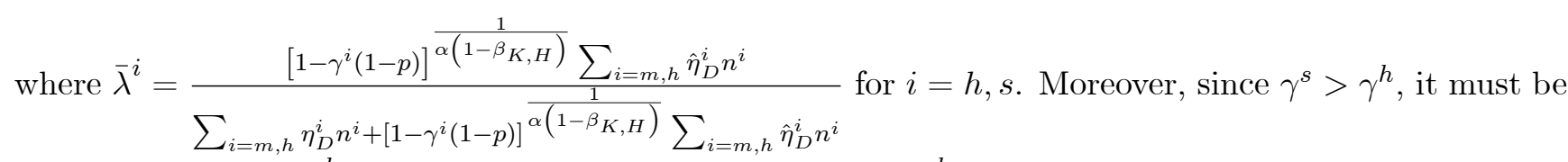
the case that $\bar{\lambda}^{s}<\bar{\lambda}^{h}$. Next, we further study $\bar{\lambda}^{s} \bar{L}<L_{p}<\bar{\lambda}^{h} \bar{L}$. First, note that $L_{p}>\bar{\lambda}^{s} \bar{L}$ if and only if $\sqrt{b^{2}-4 a c}<-2 a \bar{\lambda}^{s} \bar{L}-b$ or, which is equivalent, $-2 a \bar{\lambda}^{s} \bar{L}-b>0$ and $a\left(\bar{\lambda}^{s} \bar{L}\right)^{2}+\bar{\lambda}^{s} \bar{L} b+c>0$. Thus, we need

$$
\frac{A(p) \alpha\left(1-\beta_{K, H}\right) \beta_{U, C} \bar{p}_{C} Q_{C}}{\bar{p}_{K} \bar{L}} \sum_{i=m, h}\left(\eta_{D}^{i}+\hat{\eta}_{D}^{i}\right) n^{i}>\left(2 \bar{\lambda}^{s}-1\right) c_{\min }
$$

and

$$
\bar{c}^{s}=\frac{A(p) \alpha\left(1-\beta_{K, H}\right) \beta_{U, C} \bar{p}_{C} Q_{C}}{\bar{p}_{K} \bar{L}}\left[\frac{\sum_{i=m, h} \hat{\eta}_{D}^{i} n^{i}}{\bar{\lambda}^{s}}-\frac{\sum_{i=m, h} \eta_{D}^{i} n^{i}}{1-\bar{\lambda}^{s}}\right]>c_{\min } .
$$

If $\bar{\lambda}^{s} \leq 1 / 2$, then the first inequality always holds. If $\bar{\lambda}^{s}>1 / 2$, then the second inequality implies the first one. Therefore, $L_{p}>\bar{\lambda}^{s} \bar{L}$ if and only if $c_{\min }<\bar{c}^{s}$.

Second, note that $L_{p}<\bar{\lambda}^{h} \bar{L}$ if and only if $\sqrt{b^{2}-4 a c}>-2 a \bar{\lambda}^{h} \bar{L}-b$ or, which is equivalent, $-2 a \bar{\lambda}^{h} \bar{L}-$ $b<0$ or $a\left(\bar{\lambda}^{h} \bar{L}\right)^{2}+\bar{\lambda}^{h} \bar{L} b+c>0$. Thus, we need

$$
\frac{A(p) \alpha\left(1-\beta_{K, H}\right) \beta_{U, C} \bar{p}_{C} Q_{C}}{\bar{p}_{K} \bar{L}} \sum_{i=m, h}\left(\eta_{D}^{i}+\hat{\eta}_{D}^{i}\right) n^{i}<\left(2 \bar{\lambda}^{h}-1\right) c_{\min }
$$

or

$$
\bar{c}^{h}=\frac{A(p) \alpha\left(1-\beta_{K, H}\right) \beta_{U, C} \bar{p}_{C} Q_{C}}{\bar{p}_{K} \bar{L}}\left[\frac{\sum_{i=m, h} \hat{\eta}_{D}^{i} n^{i}}{\bar{\lambda}^{h}}-\frac{\sum_{i=m, h} \eta_{D}^{i} n^{i}}{1-\bar{\lambda}^{h}}\right]<c_{\min }
$$

If $\bar{\lambda}^{h} \leq 1 / 2$, then the first inequality never holds. If $\bar{\lambda}^{h}>1 / 2$, then the second inequality is weaker than the first one. Therefore, $L_{p}<\bar{\lambda}^{h} \bar{L}$ if and only if $c_{\min }>\bar{c}^{h}$.

Regarding occupational choices, analogous to the proof of Lemma 1, $\left[1-\gamma^{l}(1-p)\right] \bar{e}^{l}<$ $\delta\left(N^{l}\right)^{-1} \sum_{i=m, h}(1-p) \gamma^{i} N^{i} \bar{e}^{i}<\left[1-\gamma^{m}(1-p)\right] \bar{e}^{m}$, implies that only agents in group $l$ prefer to become criminals. This completes the proof of Part 2a.

Part 2b. Suppose that $L_{p}<\bar{\lambda}^{s} \bar{L}$, or which is equivalent, $c_{\min }>\bar{c}^{s}$. Then agents in group $s$ are not willing to reside in a neighborhood with private protection. Therefore, there is no private protection and the dispersed equilibrium persists. 
Proof of Lemma 4. We search for an equilibrium in which $N\left(J_{u n}, J_{u n}\right)=\left[e_{L}, e_{C}^{w}\right)$, $N\left(w, J_{u n}\right)=\left[e_{C}^{w}, e_{C}^{u n}\right)$, and $N\left(w, J_{p}\right)=\left[e_{C}^{u n}, e_{H}\right]$, where $\bar{e}>e_{C}^{u n}>e_{C}^{w}>e_{L}$. Introducing the income levels into the housing markets clearing condition we obtain the equilibrium land prices in unprotected and protected neighborhoods: $\frac{p_{L u n} \bar{L}_{u n}}{1-\beta_{K, H}}=\alpha\left[\bar{p}_{C} \int_{e_{C}^{w}}^{e_{C}^{u n}} \eta_{C}(e) e f(e) d e\right]$ and $\frac{p_{L_{p}} \bar{L}_{p}}{1-\beta_{K, H}}=$ $\alpha\left[\bar{p}_{C} \int_{e_{C}^{u n}}^{e^{H}} e f(e) d e+\left(p_{L_{u n}} \bar{L}_{u n}+p_{L_{p}} \bar{L}_{p}\right)\right]$, respectively, where $\eta_{C}(e)=1-\gamma(e)+\delta \gamma(e)$. In order to determine the equilibrium values of $e_{C}^{w}$ and $e_{C}^{u n}$, note that, in equilibrium, the agent with $e=e_{C}^{w}$ must be indifferent between being a criminal and a worker. Therefore, $\epsilon\left(e_{C}^{w}\right) \delta \int_{e_{C}^{w}}^{e_{C}^{u n}} \gamma(e) e f(e) d e=e_{C}^{w}\left(1-\gamma\left(e_{C}^{w}\right)\right)$, where $\epsilon\left(e_{C}^{w}\right)=\epsilon\left[\int_{e_{L}}^{e_{C}^{w}} f(e) d e\right]^{-1}+(1-\epsilon) e\left[\int_{e_{L}}^{e_{C}^{w}} e f(e) d e\right]^{-1}$. Due to Assumption 1.C, $e(1-\gamma(e))$ is increasing in $e$. Hence, agents with $e \in\left[e_{L}, e_{C}^{w}\right)$ prefer to be criminals, while agents with $e \in\left[e_{C}^{w}, e_{C}^{u n}\right)$ prefer to be workers. In equilibrium, the agent with $e=e_{C}^{u n}$ must be indifferent between residing in a neighborhood in $J_{u n}$ and a neighborhood in $J_{p}$. Therefore, $1-\gamma\left(e_{C}^{u n}\right)=\left(p_{L_{u n}} / p_{L_{p}}\right)^{\alpha\left(1-\beta_{K, H}\right)}$. Since $\gamma(e)$ is increasing in $e$, agents with $e \in\left[e_{C}^{u n}, e_{H}\right]$ prefer to reside in a neighborhood in $J_{p}$, while agents with $e \in\left[e_{C}^{w}, e_{C}^{u n}\right)$, prefer to reside in a neighborhood in $J_{u n}$. That's because, in equilibrium, $p_{L_{u n}}<p_{L_{p}}$, agents with $e \in\left[e_{L}, e_{C}^{w}\right)$ also prefer to reside in a neighborhood in $J_{u n}$. It remains only to prove that there is at least one vector $\left(e_{C}^{w}, e_{C}^{u n}\right)$ with $\bar{e}>e_{C}^{u n}>e_{C}^{w}>e_{L}$ that satisfies:

$$
\begin{aligned}
& \frac{\left[1-\gamma\left(e_{C}^{u n}\right)\right]^{\frac{1}{\alpha\left(1-\beta_{K, H}\right)}} \int_{e_{C}^{u n}}^{e^{H}} e f(e) d e}{\left[1-\alpha\left(1-\beta_{K, H}\right)\right] \int_{e_{C}^{w}}^{e^{u n}} \eta_{C}(e) e f(e) d e}+\frac{\alpha\left(1-\beta_{K, H}\right)}{1-\alpha\left(1-\beta_{K, H}\right)}-\frac{\bar{L}_{p}}{\bar{L}_{u n}}=0, \\
& \frac{e_{C}^{w}\left(1-\gamma\left(e_{C}^{w}\right)\right)}{\epsilon\left(e_{C}^{w}\right)}-\delta \int_{e_{C}^{w}}^{e^{u n}} \gamma(e) e f(e) d e=0 .
\end{aligned}
$$

Assume that $\bar{L}_{p} / \bar{L}_{u n}>\alpha\left(1-\beta_{K, H}\right) /\left[1-\alpha\left(1-\beta_{K, H}\right)\right]$. Then, the first equation defines a continuous function $e_{C}^{u n}=f^{1}\left(e_{C}^{w}\right)$ for each $e_{C}^{w} \in\left[e^{L}, e^{H}\right]$. Moreover, $f^{1}$ has the following properties:

- $f^{1}\left(e^{L}\right) \in\left(e^{L}, e^{H}\right)$.

- $f^{1}\left(e^{H}\right)=e^{H}$.

- $f^{1}$ is strictly increasing since

$$
\frac{d f^{1}\left(e_{C}^{w}\right)}{d e_{C}^{w}}=\frac{\eta_{C}\left(e_{C}^{w}\right) e_{C}^{w} f\left(e_{C}^{w}\right)}{\frac{\gamma^{\prime}\left(e_{C}^{u n}\right) \int_{e_{C}^{w}}^{e^{u n}} \eta_{C}(e) e f(e) d e}{\left[\alpha\left(1-\beta_{K, H}\right)\right]\left[1-\gamma\left(e_{C}^{u n}\right)\right]}+\frac{e_{C}^{u n} f\left(e_{C}^{u n}\right) \int_{e_{C}^{w}}^{e^{u n}} \eta_{C}(e) e f(e) d e}{\int_{e_{C}^{u n}}^{e^{H}} e f(e) d e}+\eta_{C}\left(e_{C}^{u n}\right) e_{C}^{u n} f\left(e_{C}^{u n}\right)}>0 .
$$


Assume that $e_{C}^{w}\left(1-\gamma\left(e_{C}^{w}\right)\right) / \epsilon\left(e_{C}^{w}\right)$ is increasing in $e_{C}^{w}$. Then, the second equation defines a continuous function $e_{C}^{w}=f^{2}\left(e_{C}^{u n}\right)$ for each $e_{C}^{u n} \in\left[e^{L}, e^{H}\right]$. Moreover, $f^{2}$ has the following properties:

- $f^{2}\left(e^{L}\right)=e^{L}$.

- $f^{2}\left(e^{H}\right) \in\left(e^{L}, e^{H}\right)$.

- $f^{2}$ is strictly increasing since $\frac{d f^{2}\left(e_{C}^{u n}\right)}{d e_{C}^{u n}}=\frac{\delta \gamma\left(e_{C}^{u n}\right) e_{C}^{u n} f\left(e_{C}^{u n}\right)}{\xi^{\prime}\left(e_{C}^{w}\right)+\delta \gamma\left(e_{C}^{w}\right) e_{C}^{w} f\left(e_{C}^{w}\right)}>0$, where $\xi\left(e_{C}^{w}\right)=$ $e_{C}^{w}\left(1-\gamma\left(e_{C}^{w}\right)\right) / \epsilon\left(e_{C}^{w}\right)$.

The properties of $f^{1}$ and $f^{2}$ imply that there exists a unique pair $\left(e_{C}^{w}, e_{C}^{u n}\right)$ such that $e_{C}^{u n}=f^{1}\left(e_{C}^{w}\right)$, $e_{C}^{w}=f^{2}\left(e_{C}^{u n}\right)$, and $e^{L}<e_{C}^{w}<e_{C}^{u n}<e^{H}$.

Proof of Lemma 5. We search for an equilibrium in which $p_{L, j}=p_{L}$ for all $j \in J$, agents with $e \in N(b)=\left[e_{L}, e_{D}^{w}\right)$ are criminals, agents with $e \in N(w)=\left[e_{D}^{w}, e_{H}\right]$ are workers, $\bar{e}>e_{D}^{w}>e_{L}$, and the proportion of agents of type $e$ in neighborhood $j$ is $\bar{L}_{j} / \bar{L}$. Introducing the income levels into the housing market clearing condition we obtain the equilibrium land price $\frac{p_{L} \bar{L}}{1-\beta_{K, H}}=\alpha\left[\int_{e^{L}}^{e^{H}} \eta_{D}(e)\left[\bar{p}_{C} e+s_{L}(e) p_{L} \bar{L}\right] f(e) d e\right]$, where $\eta_{D}(e)=1-(1-\delta)(1-p) \gamma(e)$. In order to determine the equilibrium value of $e_{D}^{w}$, note that, in equilibrium, the agent with $e=e_{D}^{w}$ must be indifferent between being a criminal and a worker. Therefore, $\bar{p}_{C} e_{D}^{w}\left[1-(1-p) \gamma\left(e_{D}^{w}\right)\right]=$ $\epsilon\left(e_{D}^{w}\right) \delta(1-p) \int_{e_{D}^{w}}^{e^{H}} \gamma(e)\left[\bar{p}_{C} e+s_{L}(e) p_{L} \bar{L}\right] f(e) d e$. Due to Assumption 1.C, $e(1-(1-p) \gamma(e))$ is increasing in $e$. Hence, agents with $e \in\left[e_{L}, e_{D}^{w}\right)$ prefer to be criminals, while agents with $e \in\left[e_{D}^{w}, e_{H}\right)$ prefer to be workers. It remains only to check that there is at least one point $e_{D}^{w}$ with $\bar{e}>e_{D}^{w}>e_{L}$ that satisfies:

$$
\frac{e_{D}^{w}\left[1-(1-p) \gamma\left(e_{D}^{w}\right)\right]}{\epsilon\left(e_{D}^{w}\right)}=\delta(1-p)\left[\begin{array}{c}
\int_{e_{D}^{w}}^{e^{H}} \gamma(e) e f(e) d e+ \\
+\frac{\alpha\left(1-\beta_{K, H}\right)\left(\int_{e^{L}}^{e^{H}} \eta_{D}(e) e f(e) d e\right)\left(\int_{e_{D}^{w}}^{e^{H}} \gamma(e) s_{L}(e) f(e) d e\right)}{\left[1-\alpha\left(1-\beta_{K, H}\right) \int_{e^{L}}^{e^{H}} \eta_{D}(e) s_{L}(e) f(e) d e\right]}
\end{array}\right]
$$

First, consider the left hand side of the equation.

- $\lim _{e_{D}^{1} \rightarrow e^{L}} \operatorname{LHS}\left(e^{L}\right)=0$

- $\operatorname{LHS}\left(e^{H}\right)=\frac{e^{H}\left[1-(1-p) \gamma\left(e^{H}\right)\right] \int_{e^{L}}^{e_{H}} e f(e) d e}{\epsilon \int_{e^{L}}^{e_{H}} e f(e) d e+e_{H}(1-\epsilon)}>0$ 
Second, consider the right hand side of the equation.

- $R H S\left(e^{L}\right)=\delta(1-p)\left[\begin{array}{c}\int_{e^{L}}^{e^{H}} \gamma(e) e f(e) d e+ \\ +\frac{\alpha\left(1-\beta_{K, H}\right)\left(\int_{e^{L}}^{e^{H}} \eta_{D}(e) e f(e) d e\right)\left(\int_{e^{L}}^{e^{H}} \gamma(e) s_{L}(e) f(e) d e\right)}{\left[1-\alpha\left(1-\beta_{K, H}\right) \int_{e^{L}}^{e^{H}} \eta_{D}(e) s_{L}(e) f(e) d e\right]}\end{array}\right]>0$

- $R H S\left(e_{D}^{w}\right)$ is strictly decreasing in $e_{D}^{w}$.

- $R H S\left(e^{H}\right)=0$.

Since $\operatorname{LHS}\left(e_{D}^{w}\right)$ and $\operatorname{RHS}\left(e_{D}^{w}\right)$ are continuous functions of $e_{D}^{w}$, there must exist $e_{D}^{w} \in\left(e^{L}, e^{H}\right)$ such that $\operatorname{LHS}\left(e_{D}^{w}\right)=\operatorname{RHS}\left(e_{D}^{w}\right)$. If, in addition, $\operatorname{LHS}\left(e_{D}^{w}\right)$ is an increasing function of $e_{D}^{w}$ (under Assumption 1.C, this holds for $\epsilon=1)$, then there is a unique $e_{D}^{w} \in\left(e^{L}, e^{H}\right)$ such that $\operatorname{LHS}\left(e_{D}^{w}\right)=R H S\left(e_{D}^{w}\right)$.

Proof of Proposition 3. Under the assumption and conditions in Lemmas 4 and 5.

Part 1 (crime). Let $C R_{C}$ and $C R_{D}$ indicate the value of the goods stolen under concentrated and dispersed protection, respectively. From Lemma $4, C R_{C}$ is given by

$$
C R_{C}=\bar{p}_{C} \delta \int_{e_{C}^{w}}^{e_{C}^{u n}} \gamma(e) e f(e) d e=\frac{\bar{p}_{C} e_{C}^{w}\left(1-\gamma\left(e_{C}^{w}\right)\right)}{\epsilon\left(e_{C}^{w}\right)} .
$$

From Lemma $5, C R_{D}$ is given by

$$
\begin{aligned}
C R_{D} & =\bar{p}_{C} \delta(1-p)\left[\begin{array}{c}
\int_{e_{D}^{w}}^{e^{H}} \gamma(e) e f(e) d e+ \\
+\frac{\alpha\left(1-\beta_{K, H}\right)\left(\int_{e^{L}}^{e^{H}} \eta_{D}(e) e f(e) d e\right)\left(\int_{e_{D}^{w}}^{e^{H}} \gamma(e) s_{L}(e) f(e) d e\right)}{\left[1-\alpha\left(1-\beta_{K, H}\right) \int_{e^{L}}^{e^{H}} \eta_{D}(e) s_{L}(e) f(e) d e\right]}
\end{array}\right] \\
& =\frac{\bar{p}_{C} e_{D}^{w}\left[1-(1-p) \gamma\left(e_{D}^{w}\right)\right]}{\epsilon\left(e_{D}^{w}\right)} .
\end{aligned}
$$

Therefore, $C R_{C}<C R_{D}$ if and only if

$$
\frac{e_{C}^{w}\left(1-\gamma\left(e_{C}^{w}\right)\right)}{\epsilon\left(e_{C}^{w}\right)}<\frac{e_{D}^{w}\left[1-(1-p) \gamma\left(e_{D}^{w}\right)\right]}{\epsilon\left(e_{D}^{w}\right)} .
$$

Part 2 (aggregate income). Let $Y_{C}$ and $Y_{D}$ denote the aggregate income under concentrated and dispersed protection, respectively. Then,

$$
\begin{aligned}
Y_{C} & =\bar{p}_{C} \int_{e_{C}^{w}}^{e_{C}^{u n}} \eta_{C}(e) e f(e) d e+\bar{p}_{C} \int_{e_{C}^{u n}}^{e^{H}} e f(e) d e+\left(p_{L_{u n}} \bar{L}_{u n}+p_{L_{p}} \bar{L}_{p}\right) \\
& =\frac{\bar{p}_{C} \int_{e_{C}^{w}}^{e^{u n}} \eta_{C}(e) e f(e) d e+\bar{p}_{C} \int_{e_{C}^{u n}}^{e^{H}} e f(e) d e}{1-\alpha\left(1-\beta_{K, H}\right)}
\end{aligned}
$$




$$
Y_{D}=\frac{\bar{p}_{C} \int_{e^{L}}^{e^{H}} \eta_{D}(e) e f(e) d e}{1-\alpha\left(1-\beta_{K, H}\right) \int_{e^{L}}^{e^{H}} \eta_{D}(e) s_{L}(e) f(e) d e}
$$

Therefore, $Y_{C}>Y_{D}$ if and only if

$$
\left(\Gamma_{C}^{u n}\right)^{\frac{1}{1-\alpha\left(1-\beta_{K, H}\right)}}+\left(\Gamma_{C}^{p}\right)^{\frac{1}{1-\alpha\left(1-\beta_{K, H}\right)}}>\left(\Gamma_{D}\right)^{\frac{1}{1-\alpha\left(1-\beta_{K, H}\right)}},
$$

where

$$
\begin{aligned}
\Gamma_{C}^{u n} & =\left[\int_{e_{C}^{w}}^{e^{u n}} \eta_{C}(e) e f(e) d e\right]^{1-\alpha\left(1-\beta_{K, H}\right)}, \\
\Gamma_{C}^{p} & =\left[\frac{\alpha\left(1-\beta_{K, H}\right) \int_{e_{C}^{w}}^{e_{C}^{u n}} \eta_{C}(e) e f(e) d e+\int_{e_{C}^{u n}}^{e^{H}} e f(e) d e}{1-\alpha\left(1-\beta_{K, H}\right)}\right]^{1-\alpha\left(1-\beta_{K, H}\right)} \\
\Gamma_{D} & =\left[\frac{\int_{e^{L}}^{e^{H}} \eta_{D}(e) e f(e) d e}{1-\alpha\left(1-\beta_{K, H}\right) \int_{e^{L}}^{e^{H}} \eta_{D}(e) s_{L}(e) f(e) d e}\right]^{1-\alpha\left(1-\beta_{K, H}\right)}
\end{aligned}
$$

Part 3 (housing prices). Suppose that protection is concentrated. Then, from Lemma 4, land prices are given by

$$
\begin{aligned}
p_{L_{u n}} & =\alpha\left(1-\beta_{K, H}\right)\left(\bar{L}_{u n}\right)^{-1} \bar{p}_{C} \int_{e_{C}^{w}}^{e^{u n}} \eta_{C}(e) e f(e) d e, \\
p_{L_{p}} & =\frac{\alpha\left(1-\beta_{K, H}\right)\left(\bar{L}_{p}\right)^{-1} \bar{p}_{C}}{1-\alpha\left(1-\beta_{K, H}\right)}\left[\int_{e_{C}^{u n}}^{e^{H}} e f(e) d e+\alpha\left(1-\beta_{K, H}\right) \int_{e_{C}^{w}}^{e_{C}^{u n}} \eta_{C}(e) e f(e) d e\right] .
\end{aligned}
$$

Suppose that protection is dispersed. Then, from Lemma 5, the price of land is given by

$$
p_{L}=\frac{\alpha\left(1-\beta_{K, H}\right) \bar{p}_{C}(\bar{L})^{-1} \int_{e^{L}}^{e^{H}} \eta_{D}(e) e f(e) d e}{1-\alpha\left(1-\beta_{K, H}\right) \int_{e^{L}}^{e^{H}} \eta_{D}(e) s_{L}(e) f(e) d e} .
$$

Therefore, $p_{L_{u n}}<p_{L}<p_{L_{p}}$ if and only if

$$
\left(\bar{L}_{u n}\right)^{-1}\left(\Gamma_{C}^{u n}\right)^{\frac{1}{1-\alpha\left(1-\beta_{K, H}\right)}}<(\bar{L})^{-1}\left(\Gamma_{D}\right)^{\frac{1}{1-\alpha\left(1-\beta_{K, H}\right)}}<\left(\bar{L}_{p}\right)^{-1}\left(\Gamma_{C}^{p}\right)^{\frac{1}{1-\alpha\left(1-\beta_{K, H}\right)}} .
$$

Part 4 (welfare). Suppose that protection is concentrated. Then, using Lemma 4, the equilibrium utility of an agent with endowment $e$ is given by 


$$
v_{C}^{e}=v \begin{cases}\frac{\epsilon\left(e_{C}^{w}\right) \delta e_{C}^{w}\left(1-\gamma\left(e_{C}^{w}\right)\right)}{\epsilon\left(e_{C}^{w}\right)\left[\frac{p_{L_{u n}}}{\alpha\left(1-\beta_{K, H}\right) \bar{p}_{C}}\right]^{\alpha\left(1-\beta_{K, H}\right)}} & e^{L} \leq e<e_{C}^{w} \\ \frac{\left[1-\gamma_{C}(e)\right] e}{\left[\frac{p_{L_{u n}}}{\alpha\left(1-\beta_{K, H}\right)^{\alpha} C}\right]^{\alpha\left(1-\beta_{K, H}\right)}} & e_{C}^{w} \leq e \leq e_{C}^{u n} \\ \frac{[1-\gamma(e)]\left[e+s_{L}(e)\left(\frac{p_{L_{u n}} \bar{L}_{u n}+p_{L_{p}} \bar{L}_{p}}{\bar{p}_{C}}\right)\right]}{\left[\frac{p_{L_{p}}}{\alpha\left(1-\beta_{K, H}\right) \bar{p}_{C}}\right]^{\alpha\left(1-\beta_{K, H}\right)}} & e_{C}^{u n} \leq e \leq e^{H}\end{cases}
$$

where $v=(\alpha)^{-\alpha}\left(\alpha \beta_{K, H}\right)^{\alpha \beta_{K, H}}\left(\bar{p}_{C} / \bar{p}_{K}\right)^{\alpha \beta_{K, H}}$. Therefore, aggregate welfare under concentrated protection is given by:

$$
W_{C}=\int_{e_{L}}^{e_{H}} v_{C}^{e} f(e) d e=v\left[\Gamma_{C}^{u n}\left(\bar{L}_{u n}\right)^{\alpha\left(1-\beta_{K, H}\right)}+\Gamma_{C}^{p}\left(\bar{L}_{p}\right)^{\alpha\left(1-\beta_{K, H}\right)}\right]
$$

Suppose that protection is dispersed. Then, using Lemma 5, the equilibrium utility of an agent with endowment $e$ is given by

$$
v_{D}^{e}=v\left\{\begin{array}{cl}
\left.\frac{\epsilon(e)\left(1-\gamma_{D}\left(e_{D}^{w}\right)\right) e_{D}^{w}}{\epsilon\left(e_{D}^{w}\right)\left[\frac{p_{L}}{\alpha\left(1-\beta_{K}, H\right.} \overline{\bar{p}}_{C}\right.}\right]^{\alpha\left(1-\beta_{K, H}\right)} & e^{L} \leq e<e_{D}^{w}, \\
\frac{\left[1-\gamma_{D}(e)\right]\left[e+s_{L}(e) \frac{p_{L} \bar{L}}{\bar{p}_{C}}\right]}{\left[\frac{p_{L}}{\alpha\left(1-\beta_{K, H}\right) \bar{p}_{C}}\right]^{\alpha\left(1-\beta_{K, H}\right)}} & e_{D}^{w} \leq e \leq e^{H} .
\end{array}\right.
$$

Therefore, aggregate welfare under dispersed protection is given by

$$
W_{D}=\int_{e_{L}}^{e_{H}} v_{D}^{e} f(e) d e=v(\bar{L})^{\alpha\left(1-\beta_{K, H}\right)} \Gamma_{D}
$$

Comparing $W_{C}$ with $W_{D}$, we have $W_{D}>W_{C}$ if and only if

$$
\Gamma_{C}^{u n}\left(\frac{\bar{L}_{u n}}{\bar{L}}\right)^{\alpha\left(1-\beta_{K, H}\right)}+\Gamma_{C}^{p}\left(\frac{\bar{L}_{p}}{\bar{L}}\right)^{\alpha\left(1-\beta_{K, H}\right)}<\Gamma_{D} .
$$

This completes the proof of Proposition 3. 